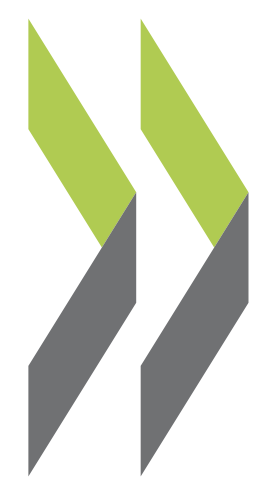

OECD Science, Technology and Industry Working Papers 1998/01

Relative Trade-Weighted

$$
\begin{array}{r}
\text { Unit Labour Costs } \\
\text { by Industry }
\end{array}
$$

Karine Lepron, Paul Schreyer 


\section{STI WORKING PAPERS}

1998/1

\section{RELATIVE TRADE-WEIGHTED UNIT LABOUR COSTS BY INDUSTRY}

Karine Lepron and Paul Schreyer 


\section{STI Working Paper Series}

The Working Papers series of the OECD Directorate for Science, Technology and Industry of the OECD is designed to make available to a wider readership selected studies prepared by staff in the Directorate or by outside consultants working on OECD projects. The papers included in the series are of a technical and analytical nature and deal with issues of data, methodology and empirical analysis in the areas of work of DSTI. The Working Papers are generally available only in their original language - English or French - with a summary in the other.

Comment on the papers is invited, and should be sent to Directorate for Science, Technology and Industry, OECD, 2 rue André-Pascal, 75775 Paris Cedex 16, France.

The opinions expressed in these papers are the sole responsibility of the author(s) and do not necessarily reflect those of the OECD or of the goverments of its Member countries.

Copyright OECD, 1998

Applications for permission to reproduce or translate all or part of this material should be made to: Head of Publication Service, OECD, 2 rue André-Pascal, 75775 Paris, Cedex 16, France. 
DSTI/DOC(98)1

\title{
RELATIVE TRADE-WEIGHTED UNIT LABOUR COSTS BY INDUSTRY
}

\author{
Karine Lepron and Paul Schreyer
}

This document presents three alternative methods for the construction of indicators of relative tradeweighted unit labour costs by industry as well as the empirical results based on these methods for 18 OECD Member countries. With some exceptions, the results show that the indicators derived from the alternative methods are highly correlated. It also turns out that there are significant variations in time profiles of relative unit labour costs by industry, an observation that underlines the usefulness of industryspecific information for an in-depth analysis of price competitiveness.

\section{COÛTS UNITAIRES RELATIFS DE LA MAIN D'OEUVRE PONDÉRÉS PAR LES ÉCHANGES, PAR BRANCHE D'INDUSTRIE}

\footnotetext{
Karine Lepron et Paul Schreyer

Ce document présente trois méthodes possibles pour la construction des indicateurs de coûts unitaires relatifs de la main-d'oeuvre pondérés par les échanges, par branche d'industrie, ainsi que les résultats empiriques basés sur ces méthodes pour 18 pays Membres de l'OCDE. Mises à part quelques exceptions, les résultats montrent que les indicateurs dérivés de ces différentes méthodes sont très corrélés. On note également des variations significatives au cours du temps des coûts unitaires relatifs de la main-d'oeuvre par branche d'industrie, une observation qui souligne l'utilité de cette information au nieveau sectoriel pour une analyse plus poussée de la compétitivité-prix.
} 


\section{RELATIVE TRADE-WEIGHTED UNIT LABOUR COSTS BY INDUSTRY}

\section{Background}

This document presents indicators of trade-weighted unit labour cost, disaggregated by detailed manufacturing industry. The indicator constitutes an addition to the OECD set of main industrial indicators and combines statistical information from different parts of the STAN family of databases.

Measures of international competitiveness have long played an important role among industrial indicators. Often, a distinction is made between price and non-price competitiveness: the first representing a firm's or industry's capacity to succeed in price competition (for a given product quality) while non-price competitiveness encompasses a host of other factors that may account for a firm's or industry's success such as product quality, diversity, novelty or after-sales services. Both dimensions are of importance, but the cost dimension is easier to measure and more often at centre-stage in policy discussions as cost competitiveness is directly influenced by macro-economic factors such as exchange rate shifts.

By definition, any measure of cost competitiveness is a relative one, relating one country's costs (or prices) in a particular industry or sector to those of its competitors. A distinction must be drawn between absolute measures of cost differences and the comparison of rates of change over time. Most empirical measures of cost competitiveness are confined to comparisons of movements in relative costs and do not allow absolute comparisons. This is essentially due to measurement problems: the most important impediment is the absence of adequate relative prices to convert measures of industry output or productivity into one common currency. The use of exchange rates or expenditure-related economy-wide purchasing power parities (PPPs) is problematic as these measures neither reflect industry differences nor necessarily relative producer prices. Although some empirical studies have taken on the issue ${ }^{1}$ and developed industry-level PPPs, they are still at an early stage and typically cover only a limited set of industries and/or countries. ${ }^{2}$

Given these difficulties, indicators are usually confined to showing relative changes of cost competitiveness. While this constitutes a drawback if the task is to rank countries by their cost competitiveness at a particular point in time, it is not a major disadvantage for other purposes, in particular for the use of this indicator to analyse trade trends. ${ }^{3}$

In what follows, cost competitiveness will be approximated through different measures of relative unit labour cost, where unit labour costs are defined as the ratio of an industry's wage bill over its volume

1. See, for example, Hooper (1996) or O’Mahony (1996).

2. In addition to questions of data availability, there are methodological issues that are still under discussion, including the techniques to apply for multilateral comparisons. For a recent discussion, see Pilat and Rao (1996).

3. This point was already made by Durand and Giorno (1987) who add: “...by restricting attention to changes in rather than levels of competitiveness, some of the biases resulting from failure to take non-price elements of competitiveness into account may be mitigated - to the extent that such non-price factors do not change rapidly or systematically when relative price competitiveness changes". 
output. Underlying such an approximation is the assumption that the movements in unit labour costs are representative for movements in total average costs. Thus, other cost elements such as the user cost of capital or intermediate products are ignored or assumed to change in parallel with the cost of labour inputs. It should also be pointed out that cost competitiveness is different from price competitiveness: only if the relation between costs and prices changes little over time, i.e., if there is a constant mark-up over costs, will cost competitiveness move in parallel with price competitiveness. This will not be the case if, for example, producers squeeze their profit margins in reaction to exchange rate appreciation and with a view to maintaining relative price competitiveness. These caveats should be kept in mind when interpreting the indicators developed in this paper.

The value-added of the present indicators lies in their industry-specificity: available measures have typically been confined to the level of total manufacturing. However, as will be shown below, industry cost competitiveness developments can vary significantly between individual industries and greater disaggregation provides useful additional information for analysis.

\section{Measuring price competitiveness}

The indicators presented here draw on the analytical framework used, for example, by the OECD in its INTERLINK model, the United States Bureau of Labor Statistics and the International Monetary Fund. Their main feature is a double-weighting procedure to reflect $i$ ) competitors' shares in export markets; and $i$ ) the relative importance of a particular market for the country and industry under consideration. The weights are applied to cost indices to form a weighted average of competitors' cost movements against which a particular country's cost changes can be compared.

Three measures of cost competitiveness are presented here. They are all based on the double-weighting procedure but they differ in the extent to which markets and competitors are covered:

- Total price competitiveness measures relative developments of unit labour costs both on a country's home market and abroad. It combines measures of import price competitiveness and export price competitiveness because on each market, unit labour cost trends of all suppliers - domestic and foreign -are taken into account. While this measure is the most comprehensive measure of price competitiveness, it is also the most demanding in terms of data needs: industry-level production data are required to weigh domestic supply and industry-level data on trade flows are required to weigh foreign supply. ${ }^{5}$

4. For a more exhaustive discussion of different measures of price competitiveness, see Durand and Giorno (1987) and Durand, Simon and Webb (1992).

5. Combining production and trade data is no trivial matter: production data often come from statistical sources that are based on activity classifications, where statistical units (establishments or firms) are grouped into industries according to their primary activity. Export and import data come from productbased statistical sources with different statistical units and classifications. To allocate product-based trade data to individual industries, a correspondence is made that links export (products) to certain industries, for example steel exports to the steel industry. This correspondence is, however, imperfect and give rise to biases. 


\section{DSTI/DOC(98)1}

- Export competitiveness measures relative developments of unit labour costs on a country's export markets. On each export market, supply of the domestic producer and of foreign producers are taken into account in the weighting scheme. Thus, unlike total price competitiveness, the export competitiveness indicator does not include import competition and permits to focus on relative unit labour cost changes abroad only. There is little difference in data requirements between the total competitiveness and the export competitiveness indicators - both require combining production and trade data at the industry level.

- Competitiveness among exporters measures relative developments of unit labour costs on a country's export markets. Unlike the two other indicators, this one only takes foreign supply on a particular market into account. The weight and development of unit labour costs of domestic suppliers in each market is ignored. This specification assumes that, on a given market, exporters only compete with other exporters, not with domestic producers, giving rise to a notion of export competitiveness in the strict sense of the term. The advantage is that only data on export flows are needed for the calculation of weights - an important issue given the numerous shortcomings of combining trade and industry data (see endnote 5).

For each country and industry, three sets of weights, one for each measure of price competitiveness, were calculated. Because weights are based on market shares, they are themselves dependent on relative competitiveness. Hence, the use of fixed weights over an extended period of time may cause biases if there are long-term movements in relative unit labour costs. Rather than using fixed weights of a given year only, the present procedure uses flexible weights that change from year to year, reflecting the evolution of export flows and hence market shares.

Each set of weights is then applied to country and industry-specific indices of unit labour costs to obtain an aggregate measure of competitors' unit labour cost developments. Changes in unit labour costs are measured as the difference between the rate of change of average compensation per employed person and the rate of change labour productivity. Average compensation is expressed in a common currency, to capture the effects of exchange rate movements. It should be noted that the use of the sets of weights is not limited to unit labour costs. An alternative usage is the application to exchange rates only, giving rise to trade-weighted exchange rates - a common indicator for the extent to which a country's foreign trade is likely to be affected by exchange rate swings. Similarly, prices could be used instead of unit labour costs if such data are available.

\section{Results}

Relative unit labour costs and their development were evaluated, according to three different methods, for 18 countries and 25 industries on a yearly basis between 1979 and 1994. The wealth of series does not permit a full reproduction of results. However, three types of tables are provided in the Annex:

- At the level of total manufacturing, a decomposition of the series of relative unit labour costs (calculated according to the first method - total price competitiveness) into its components: average compensation per employed person, exchange rate movements and labour productivity growth (Annex Tables 1 to 18 ). 
- At the industry level, Annex Tables 19 to 54 spell out the series of industry-specific tradeweighted unit labour costs for each country, calculated according to the first method - total price competitiveness, and according to the third method - competitiveness among exporters.

These tables give rise to several observations:

- At the level of total manufacturing, the trends emerging from the three OECD methods are quite similar, as illustrated in Figure 1 that compares results for Japan, France, the United Kingdom and the United States. For purposes of comparison, Figure 1 also plots the relative unit labour cost series from a different source, the United States Bureau of Labor Statistics (BLS). This comparison is only possible at the level of total manufacturing and for a limited number of countries. For most of them, trends are highly correlated, but exceptions can be found, especially among European countries. Differences can be due to different measures of unit labour costs (BLS features a preferred measure based on hourly compensation while OECD data is limited to compensation per employee) or to differences in the weighting scheme (the set of countries varies between BLS and OECD and BLS employs a fixed weighting pattern whereas the OECD indicators are based on annually changing weights).

- Regarding the comparison of the different methods, correlation coefficients were constructed to test whether the methods yield widely diverging results. Annex Table 56 shows how relative unit labour cost trends calculated with the first methodology (total price competitiveness) compares with the trends based on the third methodology (competitiveness among exporters). As it turns out, the series are highly correlated for most countries and industries. There are, however, exceptions. Canada is a case in point where correlation between the results of the two methods tends to be weak for some industries. To explain this divergence, note the exceptional importance of the United States as a market in the Canadian export composition. Add to this that the main difference between the two measures of relative unit labour costs is the inclusion (exclusion) of domestic suppliers on various export markets. Because domestic supply carries a large weight in most industries in the United States, this produces a marked difference between the two methods for the US market. This is true for all featuring countries, but receives the largest weight in the Canadian case because of the importance of the US market in Canadian exports.

- The conclusion is that the easy-to-calculate third method (competitiveness among exporters) goes a long way in depicting trends of industry-level relative unit labour costs that are reasonably close to the measure of total price competitiveness that is the theoretically preferred measure. However, for countries whose export market structure is highly concentrated in large markets (where domestic suppliers tend to be important) the measure of competitiveness among exporters may prove an insufficient approximation to total price competitiveness. 


\section{ANNEX: RELATIVE TRADE-WEIGHTED UNIT LABOUR COSTS BY INDUSTRY: METHODOLOGY}

\section{Types of competition}

The following methodological description relates to the derivation of relative unit labour cost measures. For notational simplicity, no industry-specific index has been introduced but it should be understood that all calculations relate to a particular industry. The theoretical basis to derive trade-weighted indicators of competitiveness has been thoroughly described in a methodological paper of the International Monetary Fund (McGuirk, 1986). It shows that, in a market with monopolistic competition with differentiated goods, the growth rate of the demand for country $j$ 's product on the geographical market $k\left(\Delta \ln D_{j}^{k}\right)$ can be expressed as a function of the rate of change of relative prices $P_{l}^{k} / P_{j}^{k}$.

$$
\Delta \ln D_{j}^{k}=\sigma \sum_{l \neq j} s_{l}^{k} \Delta \ln \left(\frac{P_{l}^{k}}{P_{j}^{k}}\right)
$$

where $\quad l=1,2, \ldots N$ competitors;

$j=1,2, \ldots N$ featuring countries;

$k=1,2, \ldots M$ geographical markets;

$s_{l}^{k}=\frac{X_{l}^{k}}{\sum_{l} X_{l}^{k}} ; X_{l}^{k}:$ country $l$ 's exports to market $k$

Each pair of relative prices $P_{l}^{k} / P_{j}^{k}$ between the featuring country $j$ and its $N-1$ competitors is weighted with the respective competitor's market share $s_{l}^{k} . \sigma$ is a constant term capturing the price elasticity of demand. Because $\sum_{l} s_{l}^{k}=\sum_{l \neq j} s_{l}^{k}+s_{j}^{k}=1$, equation (1) can be rewritten as:

$$
\begin{aligned}
& \Delta \ln D_{j}^{k}=\sigma\left\{\sum_{l \neq j} s_{l}^{k} \Delta \ln P_{l}^{k}-\left(1-s_{j}^{k}\right) \Delta \ln P_{j}^{k}\right\} \\
& \text { or } \Delta \ln D_{j}^{k}=\sigma\left(1-s_{j}^{k}\right)\left\{\sum_{l \neq j} \frac{s_{l}^{k}}{1-s_{j}^{k}} \Delta \ln P_{l}^{k}-\Delta \ln P_{j}^{k}\right\}
\end{aligned}
$$

The expression in brackets on the right hand side of (2) is the starting point for the competitiveness indicator. It corresponds to the difference in price movements of the featuring country, $\Delta \ln P_{j}^{k}$, and a weighted average of those of its competitors: $\Delta \ln P C_{j}^{k}=\sum_{l \neq j} \frac{s_{l}^{k}}{1-s_{j}^{k}} \Delta \ln P_{l}^{k}$. The division by $\left(1-s_{j}^{k}\right)$ normalises market shares so that for each featuring country $j$, the set of $N-l$ competitors $l$ 
$(l=1,2, \ldots j-1, j+1, \ldots N)$ equals 100 percent. The featuring country is excluded from the set of competitors as it is obviously not in competition with itself. ${ }^{6}$

As a next step, normalised demand for the featuring country's product on the $k$ different markets is aggregated to yield the overall changes in demand for $j$ 's product, $\Delta \ln D_{j}$. Price changes in each market $k$ enter with the weight $w_{j}^{k}$ the share that each market $k$ occupies in country $j$ 's sales. It should be noted that this aggregation (equation (3)) includes country $j$ 's home market where it competes with imports. In this sense, it describes price competition on the home market and on export markets which will be captured by a measure of total price competitiveness, combining measures of import and export price competitiveness.

$\Delta \ln D_{j}=\sum_{k} w_{j}^{k}\left(\frac{\Delta \ln D_{j}^{k}}{\sigma\left(1-s_{j}^{k}\right)}\right)$

where: $w_{j}^{k}=\frac{X_{j}^{k}}{\sum_{k} X_{j}^{k}}$

If the indicator is limited to export competition, aggregation is carried out over export markets only, excluding the featuring country's home market [equation (4)]:

$\Delta \ln \widetilde{D}_{j}=\sum_{k \neq j} \widetilde{w}_{j}^{k}\left(\frac{\Delta \ln \widetilde{D}_{j}^{k}}{\sigma\left(1-s_{j}^{k}\right)}\right)$

where $\tilde{w}_{j}^{k}=\frac{X_{j}^{k}}{\sum_{k \neq j} X_{j}^{k}}$

Implicit in the notions of total and export competition is the assumption that on every market, domestic suppliers compete with importers. In contrast, the more narrow notion of competition among exporters only considers foreign suppliers on a market. It assumes a two stage-decision process: in a first stage, consumers decide on the overall spending on imported goods and in a second stage, they allocate this expenditure to the different imported goods. The implication is that the overall volume of imports to every market can be considered pre-determined and there is no direct price competition between domestic products and imported ones. Unsurprisingly, the adoption of this hypothesis can significantly change the weights of competitor countries. In this case of competition among exporters, equation (1) reads as:

$$
\Delta \ln \widetilde{\widetilde{D}}_{j}^{k}=\sigma \sum_{l \neq j} \tilde{s}_{l}^{k} \Delta \ln \left(\frac{P_{l}^{k}}{P_{j}^{k}}\right)
$$

6. One consequence is that weights attached to competing countries on third markets depend on the featured country. It should also be noted that the information incorporated in $\left(1-s_{j}^{k}\right)$, i.e., the share that the featuring country's competitors as a whole occupy in the market, is lost from the calculation. Alternatively, it could be argued that a competitor's sales are determined by the price that he charges relative to the average market price. In this case, the featuring country's price would be part of the average market price, defined as $\sum_{l} s_{l}^{k} \Delta \ln P_{l}^{k}$, and no normalisation would be requested. However, in the present context, normalisation is retained. 
where $\tilde{s}_{l}^{k}=\frac{X_{l}^{k}}{\sum_{l \neq k} X_{l}^{k}} ; \tilde{s}_{k}^{k}=0$.

The share of the domestic supplier, $s_{k}^{k}$, is set to equal zero, thus limiting the set of competitors to those who export to market $k$. Normalisation and aggregation is as before and yields:

$\Delta \ln \widetilde{\widetilde{D}}_{j}=\sum_{k \neq j} \widetilde{w}_{j}^{k}\left(\frac{\Delta \ln \widetilde{\widetilde{D}}_{j}^{k}}{\sigma\left(1-\tilde{s}_{j}^{k}\right)}\right)$

where $\tilde{w}_{j}^{k}=\frac{X_{j}^{k}}{\sum_{k \neq j} X_{j}^{k}}$

\section{Competitors' weights}

Based on these notions of competition, three weighting schemes can be derived as a basis for measures of price competitiveness. Starting with the measure of total price competitiveness, equation (3) can be written as:

$$
\Delta \ln D_{j}=\sum_{k} w_{j}^{k}\left(\sum_{l \neq k} \frac{s_{l}^{k}}{1-s_{j}^{k}} \Delta \ln P_{l}^{k}-\Delta \ln P_{j}^{k}\right)
$$

An important simplification for an empirical implementation consists in assuming that if a competitor country changes the price of his product, this price change applies to all its (export) markets. Formally, this amounts to stating $\Delta \ln P_{l}^{k}=\Delta \ln P_{l}$ and equation (7) now reads as:

$$
\Delta \ln D_{j}=\sum_{l \neq j} \sum_{k} w_{j}^{k} \frac{s_{l}^{k}}{1-s_{j}^{k}} \Delta \ln P_{l}-\Delta \ln P_{j}
$$

From equation (8) it is possible to identify, for each featuring country $j$, a set of weights, $T W_{j}$ with elements $T W_{j, l}$, one for each featuring country $j$ and its competitors' price change $\Delta \ln P_{l}$. Similarly, weights $T \tilde{W}_{j}$ and $T \tilde{\tilde{W}}_{j}$ can be derived as bases for measures of export competitiveness and competitiveness among exporters.

Table 1 shows the full definitions.

Empirically, weights are calculated for every year in the period under consideration. The advantage of flexible weights is that they allow for changes in the structure of foreign trade and competition - changes which may arise as a consequence of changes in competitiveness. A selection of weights at the level of total manufacturing is shown in Annex Tables 1 to 18. As it turns out, they are both sensitive to the type of competitiveness indicator and to the underlying year. 
Table 1. Competitors' weights

\begin{tabular}{lll}
\hline $\begin{array}{l}\text { Weights for competitors } l \text { to measure } \\
\text { country } j \text { ' } s \text {. }\end{array}$ & Weight & Definition \\
\hline Total price competitiveness & $T W_{j, l}$ & $\sum_{k} w_{j}^{k} s_{l}^{k} /\left(1-s_{j}^{k}\right)$ \\
& $T \tilde{W}_{j, l}$ & $\sum_{k \neq j}^{k} \tilde{w}_{j}^{k} s_{l}^{k} /\left(1-s_{j}^{k}\right)$ \\
Export price competitiveness & $\tilde{\tilde{W}}_{j, l}$ & $\sum_{k \neq j} \tilde{w}_{j}^{k} \tilde{s}_{l}^{k} /\left(1-\tilde{s}_{j}^{k}\right)$ \\
\hline
\end{tabular}

\section{Trade-weighted relative unit labour costs}

As a final step, indicators of competitiveness are derived by substituting rates of change in unit labour costs for the changes in prices. Thus, $\Delta \ln P_{l}$ is replaced by $\Delta \ln U C L_{l}$ where $U C L_{l}=\frac{w_{l} L_{l}}{Q_{l}} e_{l} . w_{l} L_{l}$ is country $l$ 's wage bill at current prices, $Q_{l}$ its constant price output (value-added), and $e_{l}$ its current exchange rate with respect to a common currency. Formulated as growth rates, the change in unit labour costs is the difference between changes in the wage rate and changes in labour productivity: $\Delta \ln U L C_{l}=\Delta \ln w_{l}-\Delta \ln \left(Q_{l} / L_{l}\right)$. The final indicators of relative trade-weighted unit labour costs $R U L C$ are as shown in Table 2.

Table 2. Relative unit labour cost: three measures

\begin{tabular}{ll}
\hline Measure of country $j$ 's: & Definition \\
\hline Total price competitiveness & $\Delta \ln R U L C_{j}=\Delta \ln U L C_{j}-\sum_{l \neq j} T W_{j, l} \Delta \ln U L C_{l} ;$ \\
Export price competitiveness & $\Delta \ln R U L C_{j}=\Delta \ln U L C_{j}-\sum_{l \neq j} T \tilde{W}_{j, l} \Delta \ln U L C_{l} ;$ \\
Price competitiveness among exporters & $\Delta \ln R U L C_{j}=\Delta \ln U L C_{j}-\sum_{l \neq j} T \tilde{\tilde{W}}_{j, l} \Delta \ln U L C_{l} ;$ \\
\hline
\end{tabular}




\section{REFERENCES}

Durand, M. and C. Giorno (1987), "Indicators of International Competitiveness: Conceptual Aspects and Evaluation", OECD Economic Studies No.9.

Durand, M., J. Simon and C. Webb (1992), "OECD's Indicators of International Trade and Competitiveness", OECD Economics Department Working Papers No 120.

Hooper, P. (1996), “Comparing Manufacturing Output Levels Among the Major Industrial Countries", in OECD (1996), Industry Productivity: International Comparisons and Measurement Issues.

OECD (1996), Technology and Industrial Performance, Paris.

O’Mahony, M. (1996), "Conversion Factors in Relative Productivity Calculations: Theory and Practice", in OECD (1996), Industry Productivity: International Comparisons and Measurement Issues.

Pilat, D. and P. Rao (1996), "Multilateral Comparisons of Output, Productivity and Purchasing Power Parities in Manufacturing", Review of Income and Wealth, June.

McGuirk, A. (1986), "Measuring Price Competitiveness for Industrial Country Trade in Manufactures", IMF Working Paper WP/87/34.

United States Department of Labor, Bureau of Labor Statistics (1996), International Comparisons of Manufacturing Productivity and Unit Labor Cost Trends. 

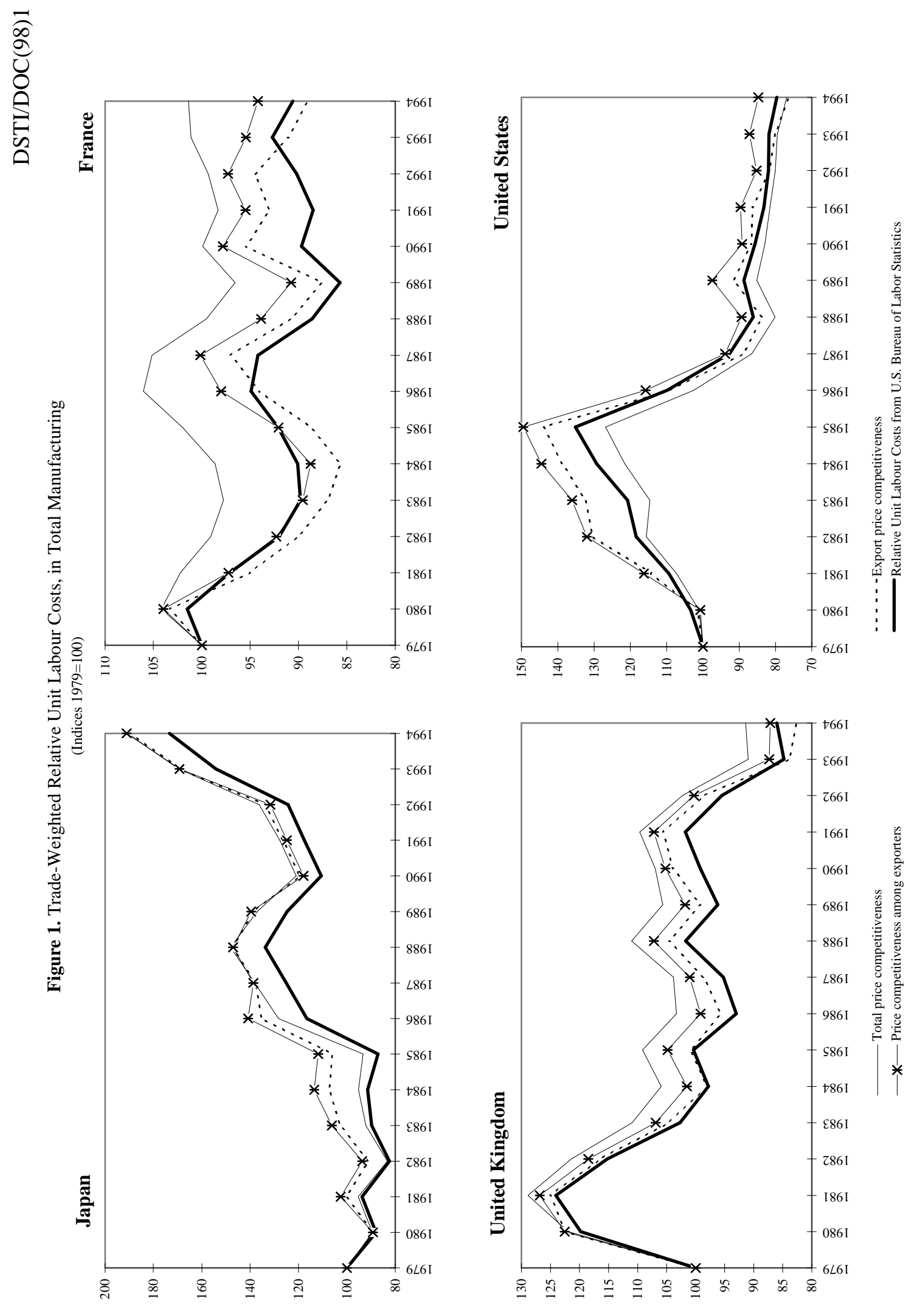
ANNEX TABLES 1-18

TRADE-WEIGHTED RELATIVE UNIT LABOUR COST IN TOTAL MANUFACTURING METHOD: TOTAL PRICE COMPETITIVENESS

Table 1. Australia

Table 2. Austria

Table 3. Belgium / Luxembourg

Table 4. Canada

Table 5. Denmark

Table 6. Finland

Table 7. France

Table 8 . Western Germany

Table 9. Greece

Table 10. Italy

Table 11. Japan

Table 12. Netherlands

Table 13. Norway

Table 14. Portugal

Table 15. Spain

Table 16. Sweden

Table 17. United Kingdom

Table 18. United States 
Trade-Weighted Output per Employed Person and Unit Labour Costs in Total Manufacturing, 1979-94 (Indexes: 1990=100)

Competitors: Austria, Belgium, Canada, Denmark, Finland, France, Germany, Greece, Italy, Japan, Netherlands, Norway, Portugal, Spain, Sweden, United Kingdom, United States

\begin{tabular}{|c|c|c|c|}
\hline \multirow{2}{*}{ Year } & \multicolumn{3}{|c|}{ Output per Employed Person } \\
& U.S. Dollar Basis \\
\cline { 2 - 4 } & Own & Competitors' & Ratio \\
\hline 1979 & 81.5 & 72.0 & 113.1 \\
1980 & 82.5 & 71.2 & 115.7 \\
1981 & 84.2 & 73.5 & 114.5 \\
1982 & 86.0 & 75.3 & 114.2 \\
1983 & 84.5 & 79.0 & 106.9 \\
1984 & 87.6 & 83.0 & 105.5 \\
1985 & 92.1 & 86.8 & 106.1 \\
1986 & 93.4 & 87.3 & 106.9 \\
1987 & 96.3 & 91.3 & 105.4 \\
1988 & 99.7 & 95.6 & 104.2 \\
1989 & 101.7 & 97.8 & 104.0 \\
1990 & 100.0 & 100.0 & 100.0 \\
1991 & 103.1 & 100.9 & 102.2 \\
1992 & 105.8 & 102.1 & 103.6 \\
1993 & 110.2 & 103.0 & 107.1 \\
1994 & 118.4 & 107.3 & 110.4 \\
\hline
\end{tabular}

\begin{tabular}{|c|r|r|r|}
\hline \multirow{2}{*}{ Year } & \multicolumn{3}{|c|}{ Unit Labour Costs } \\
& \multicolumn{3}{|c|}{ National Currency Basis } \\
\cline { 2 - 4 } & $\begin{array}{c}\text { Own } \\
\text { Index }\end{array}$ & $\begin{array}{c}\text { Competitors' } \\
\text { Index }\end{array}$ & Ratio \\
\hline 1979 & 51.2 & 71.3 & 71.9 \\
1980 & 54.5 & 79.5 & 68.5 \\
1981 & 61.5 & 85.3 & 72.1 \\
1982 & 68.7 & 89.2 & 76.9 \\
1983 & 76.9 & 89.8 & 85.6 \\
1984 & 78.2 & 90.8 & 86.1 \\
1985 & 79.9 & 91.5 & 87.3 \\
1986 & 84.4 & 95.5 & 88.5 \\
1987 & 86.7 & 94.9 & 91.4 \\
1988 & 88.2 & 94.4 & 93.5 \\
1989 & 91.4 & 96.5 & 94.7 \\
1990 & 100.0 & 100.0 & 100.0 \\
1991 & 102.3 & 104.2 & 98.1 \\
1992 & 102.4 & 107.5 & 95.3 \\
1993 & 101.6 & 109.8 & 92.5 \\
1994 & 97.3 & 108.9 & 89.4 \\
\hline
\end{tabular}

\begin{tabular}{|c|r|c|c|}
\hline \multirow{2}{*}{ Year } & \multicolumn{3}{|c|}{ Unit Labour Costs } \\
& \multicolumn{3}{|c|}{ U.S. Dollar Basis } \\
\cline { 2 - 4 } & Own & Competitors' & Ratio \\
\hline 1979 & 73.3 & 66.6 & 110.2 \\
1980 & 79.5 & 74.4 & 106.9 \\
1981 & 90.5 & 73.7 & 122.8 \\
1982 & 89.2 & 71.4 & 124.9 \\
1983 & 88.7 & 69.4 & 127.8 \\
1984 & 88.0 & 67.5 & 130.3 \\
1985 & 71.5 & 67.2 & 106.5 \\
1986 & 72.3 & 83.4 & 86.7 \\
1987 & 77.8 & 91.4 & 85.2 \\
1988 & 88.3 & 96.4 & 91.6 \\
1989 & 92.5 & 93.9 & 98.5 \\
1990 & 100.0 & 100.0 & 100.0 \\
1991 & 102.1 & 105.6 & 96.6 \\
1992 & 96.4 & 112.3 & 85.8 \\
1993 & 88.5 & 116.1 & 76.2 \\
1994 & 91.2 & 119.5 & 76.3 \\
\hline
\end{tabular}

\begin{tabular}{|c|r|c|r|}
\hline \multirow{2}{*}{ Year } & \multicolumn{3}{|c|}{$\begin{array}{c}\text { Exchange } \\
\text { Rates }\end{array}$} \\
\cline { 2 - 4 } & $\begin{array}{c}\text { Own } \\
\text { Index }\end{array}$ & $\begin{array}{c}\text { Competitors' } \\
\text { Index }\end{array}$ & Ratio \\
\hline 1979 & 69.8 & 108.7 & 64.3 \\
1980 & 68.6 & 110.8 & 61.9 \\
1981 & 67.9 & 118.9 & 57.1 \\
1982 & 77.0 & 130.9 & 58.8 \\
1983 & 86.6 & 134.3 & 64.5 \\
1984 & 89.0 & 140.7 & 63.2 \\
1985 & 111.8 & 143.5 & 77.9 \\
1986 & 116.8 & 115.8 & 100.8 \\
1987 & 111.5 & 104.2 & 107.0 \\
1988 & 99.9 & 98.7 & 101.2 \\
1989 & 98.7 & 103.4 & 95.4 \\
1990 & 100.0 & 100.0 & 100.0 \\
1991 & 100.2 & 98.8 & 101.5 \\
1992 & 106.3 & 96.1 & 110.6 \\
1993 & 114.8 & 97.8 & 117.4 \\
1994 & 106.8 & 95.9 & 111.4 \\
\hline
\end{tabular}

\begin{tabular}{|l|c|c|}
\cline { 2 - 3 } \multicolumn{1}{c|}{} & \multicolumn{2}{c|}{ Trade Weights } \\
\cline { 2 - 3 } \multicolumn{1}{c|}{} & 1970 & 1994 \\
\hline Australia & - & - \\
Austria & 0.4 & 0.6 \\
Belgium & 0.9 & 1.6 \\
Canada & 5.5 & 2.0 \\
Denmark & 0.4 & 0.6 \\
Finland & 0.6 & 1.2 \\
France & 2.5 & 3.8 \\
Western Germany & 8.9 & 9.8 \\
Greece & 0.2 & 0.1 \\
Italy & 3.0 & 4.6 \\
Japan & 20.4 & 31.6 \\
Netherlands & 1.9 & 1.8 \\
Norway & 0.3 & 0.3 \\
Portugal & 0.1 & 0.2 \\
Spain & 0.5 & 0.9 \\
Sweden & 2.1 & 2.6 \\
United Kingdom & 24.3 & 9.2 \\
United States & 28.0 & 29.3 \\
\hline
\end{tabular}


Annex Table 2

Trade-Weighted Output per Employed Person and Unit Labour Costs in Total Manufacturing, 1979-94 (Indexes: 1990=100)

Competitors: Australia, Belgium, Canada, Denmark, Finland, France, Germany, Greece, Italy, Japan, Netherlands, Norway, Portugal, Spain, Sweden, United Kingdom, United States

\begin{tabular}{|c|c|c|c|}
\hline \multirow{2}{*}{ Year } & \multicolumn{3}{|c|}{ Output per Employed Person } \\
\cline { 2 - 4 } & Own & Competitors' & Ratio \\
\cline { 2 - 4 } & Index & 79.6 & 86.4 \\
1979 & 68.7 & 78.5 & 89.5 \\
1980 & 70.2 & 79.6 & 87.9 \\
1981 & 70.0 & 80.5 & 90.7 \\
1982 & 72.9 & 84.5 & 90.8 \\
1983 & 76.8 & 88.1 & 90.2 \\
1984 & 79.5 & 90.7 & 91.3 \\
1985 & 82.9 & 91.4 & 91.4 \\
1986 & 83.6 & 91.8 & 92.3 \\
1987 & 84.7 & 95.5 & 96.8 \\
1988 & 92.5 & 98.0 & 98.1 \\
1989 & 96.1 & 100.0 & 100.0 \\
1990 & 100.0 & 101.6 & 101.3 \\
1991 & 103.0 & 102.2 & 103.9 \\
1992 & 106.2 & 102.0 & 105.3 \\
1993 & 107.4 & 109.0 & 105.7 \\
1994 & 115.2 &
\end{tabular}

\begin{tabular}{|c|r|c|c|}
\hline \multirow{2}{*}{ Year } & \multicolumn{3}{|c|}{ Unit Labour Costs } \\
& \multicolumn{3}{|c|}{ National Currency Basis } \\
\cline { 2 - 4 } & Own & Competitors' & Ratio \\
\hline 1979 & 76.9 & 65.2 & 118.0 \\
1980 & 82.0 & 72.2 & 113.5 \\
1981 & 86.6 & 77.0 & 112.5 \\
1982 & 91.4 & 82.0 & 111.5 \\
1983 & 92.5 & 83.0 & 111.5 \\
1984 & 92.1 & 84.6 & 108.8 \\
1985 & 95.2 & 87.0 & 109.4 \\
1986 & 98.4 & 90.2 & 109.1 \\
1987 & 101.1 & 94.2 & 107.4 \\
1988 & 96.0 & 94.4 & 101.7 \\
1989 & 96.5 & 96.3 & 100.2 \\
1990 & 100.0 & 100.0 & 100.0 \\
1991 & 104.2 & 104.4 & 99.8 \\
1992 & 109.5 & 109.5 & 99.9 \\
1993 & 113.8 & 112.8 & 100.8 \\
1994 & 112.2 & 109.9 & 102.1 \\
\hline
\end{tabular}

\begin{tabular}{|c|r|c|r|}
\hline \multirow{2}{*}{ Year } & \multicolumn{3}{|c|}{ Unit Labour Costs } \\
& \multicolumn{3}{|c|}{ U.S. Dollar Basis } \\
\cline { 2 - 4 } & Own & Competitors' & \multicolumn{1}{c|}{ Ratio } \\
\hline 1979 & 65.4 & 64.9 & 100.8 \\
1980 & 72.0 & 72.3 & 99.7 \\
1981 & 61.8 & 63.3 & 97.6 \\
1982 & 60.9 & 60.9 & 99.9 \\
1983 & 58.6 & 57.4 & 102.1 \\
1984 & 52.3 & 53.2 & 98.4 \\
1985 & 52.3 & 52.9 & 98.8 \\
1986 & 73.3 & 70.3 & 104.3 \\
1987 & 91.0 & 86.0 & 105.8 \\
1988 & 88.4 & 88.7 & 99.7 \\
1989 & 82.9 & 85.0 & 97.5 \\
1990 & 100.0 & 100.0 & 100.0 \\
1991 & 101.5 & 102.3 & 99.3 \\
1992 & 113.2 & 112.3 & 100.8 \\
1993 & 111.2 & 107.1 & 103.8 \\
1994 & 111.7 & 105.9 & 105.4 \\
\hline
\end{tabular}

\begin{tabular}{|c|c|c|c|}
\hline \multirow{2}{*}{ Year } & \multicolumn{3}{|c|}{$\begin{array}{c}\text { Exchange } \\
\text { Rates }\end{array}$} \\
\cline { 2 - 4 } & Own & $\begin{array}{c}\text { Competitors' } \\
\text { Index }\end{array}$ & Ratio \\
\hline 1979 & 117.6 & 101.0 & 116.4 \\
1980 & 113.8 & 100.9 & 112.8 \\
1981 & 140.1 & 123.6 & 113.4 \\
1982 & 150.0 & 136.4 & 110.0 \\
1983 & 158.0 & 146.5 & 107.9 \\
1984 & 176.0 & 162.3 & 108.5 \\
1985 & 182.0 & 168.2 & 108.2 \\
1986 & 134.3 & 129.1 & 104.0 \\
1987 & 111.2 & 109.7 & 101.4 \\
1988 & 108.6 & 106.7 & 101.8 \\
1989 & 116.4 & 113.6 & 102.4 \\
1990 & 100.0 & 100.0 & 100.0 \\
1991 & 102.7 & 102.1 & 100.5 \\
1992 & 96.7 & 97.7 & 99.0 \\
1993 & 102.3 & 106.9 & 95.7 \\
1994 & 100.5 & 105.8 & 95.0 \\
\hline
\end{tabular}

\begin{tabular}{|l|c|c|}
\cline { 2 - 3 } \multicolumn{1}{c|}{} & \multicolumn{2}{c|}{ Trade Weights } \\
\cline { 2 - 3 } \multicolumn{1}{c|}{} & 1970 & 1994 \\
\hline Australia & 0.2 & 0.2 \\
Austria & - & - \\
Belgium & 2.6 & 3.3 \\
Canada & 0.6 & 0.6 \\
Denmark & 1.9 & 1.0 \\
Finland & 1.0 & 0.9 \\
France & 5.4 & 7.2 \\
Western Germany & 54.3 & 52.6 \\
Greece & 0.3 & 0.4 \\
Italy & 9.2 & 11.2 \\
Japan & 1.8 & 4.3 \\
Netherlands & 2.8 & 3.6 \\
Norway & 0.8 & 0.5 \\
Portugal & 0.7 & 0.5 \\
Spain & 0.6 & 1.9 \\
Sweden & 4.4 & 2.1 \\
United Kingdom & 9.2 & 4.5 \\
United States & 4.3 & 5.2 \\
\hline
\end{tabular}


Trade-Weighted Output per Employed Person and Unit Labour Costs in Total Manufacturing, 1979-94 (Indexes: 1990=100)

Competitors: Australia, Austria, Canada, Denmark, Finland, France, Germany, Greece, Italy, Japan, Netherlands, Norway, Portugal, Spain, Sweden, United Kingdom, United States

\begin{tabular}{|c|c|c|c|}
\hline \multirow{2}{*}{ Year } & \multicolumn{3}{|c|}{ Output per Employed Person } \\
\cline { 2 - 4 } & Own & Competitors' & \\
& Index & Index & Ratio \\
\hline 1979 & 61.5 & 76.7 & 80.1 \\
1980 & 64.6 & 75.8 & 85.2 \\
1981 & 68.3 & 77.5 & 88.1 \\
1982 & 73.6 & 78.9 & 93.3 \\
1983 & 79.5 & 83.1 & 95.6 \\
1984 & 83.1 & 86.5 & 96.0 \\
1985 & 85.8 & 89.0 & 96.4 \\
1986 & 87.5 & 90.1 & 97.1 \\
1987 & 89.1 & 91.3 & 97.6 \\
1988 & 94.3 & 95.6 & 98.6 \\
1989 & 98.5 & 98.4 & 100.1 \\
1990 & 100.0 & 100.0 & 100.0 \\
1991 & 101.1 & 101.1 & 100.0 \\
1992 & 102.7 & 102.4 & 100.3 \\
1993 & 103.5 & 103.0 & 100.5 \\
1994 & 111.9 & 109.4 & 102.2 \\
\hline
\end{tabular}

\begin{tabular}{|c|c|c|c|}
\hline \multirow{2}{*}{ Year } & \multicolumn{3}{|c|}{ Unit Labour Costs } \\
& \multicolumn{3}{|c|}{ National Currency Basis } \\
\cline { 2 - 4 } & $\begin{array}{c}\text { Own } \\
\text { Index }\end{array}$ & $\begin{array}{c}\text { Competitors' } \\
\text { Index }\end{array}$ & Ratio \\
\hline 1979 & 83.8 & 66.4 & 126.3 \\
1980 & 88.0 & 73.3 & 120.1 \\
1981 & 88.2 & 78.4 & 112.5 \\
1982 & 88.1 & 83.5 & 105.4 \\
1983 & 87.3 & 84.6 & 103.3 \\
1984 & 90.2 & 86.3 & 104.6 \\
1985 & 93.3 & 88.9 & 105.0 \\
1986 & 94.6 & 92.0 & 102.8 \\
1987 & 96.6 & 95.2 & 101.4 \\
1988 & 94.9 & 94.7 & 100.2 \\
1989 & 95.3 & 96.0 & 99.3 \\
1990 & 100.0 & 100.0 & 100.0 \\
1991 & 106.2 & 104.8 & 101.3 \\
1992 & 109.9 & 108.8 & 101.0 \\
1993 & 111.2 & 111.3 & 99.9 \\
1994 & 107.2 & 108.8 & 98.5 \\
\hline
\end{tabular}

\begin{tabular}{|c|c|c|c|}
\hline \multirow[t]{2}{*}{ Year } & \multicolumn{3}{|c|}{$\begin{array}{l}\text { Unit Labour Costs } \\
\text { U.S. Dollar Basis }\end{array}$} \\
\hline & $\begin{array}{l}\text { Own } \\
\text { Index }\end{array}$ & $\begin{array}{l}\text { Competitors' } \\
\text { Index }\end{array}$ & Ratio \\
\hline 1979 & 95.5 & 68.3 & 139.9 \\
\hline 1980 & 100.6 & 77.1 & 130.4 \\
\hline 1981 & 79.3 & 69.0 & 115.1 \\
\hline 1982 & 64.4 & 66.3 & 97.2 \\
\hline 1983 & 57.1 & 61.9 & 92.2 \\
\hline 1984 & 52.2 & 57.8 & 90.3 \\
\hline 1985 & 52.5 & 57.7 & 90.9 \\
\hline 1986 & 70.8 & 73.6 & 96.1 \\
\hline 1987 & 86.5 & 87.5 & 98.9 \\
\hline 1988 & 86.2 & 89.7 & 96.1 \\
\hline 1989 & 80.8 & 85.9 & 94.2 \\
\hline 1990 & 100.0 & 100.0 & 100.0 \\
\hline 1991 & 103.9 & 102.8 & 101.0 \\
\hline 1992 & 114.2 & 111.2 & 102.7 \\
\hline 1993 & 107.4 & 105.7 & 101.6 \\
\hline 1994 & 107.1 & 105.0 & 101.9 \\
\hline
\end{tabular}

\begin{tabular}{|c|r|c|r|}
\hline \multirow{2}{*}{ Year } & \multicolumn{3}{|c|}{ Exchange } \\
& Rates \\
\cline { 2 - 4 } & Own & Competitors' & Ratio \\
\hline 1979 & 87.7 & 97.3 & 90.2 \\
1980 & 87.5 & 96.0 & 91.2 \\
1981 & 111.1 & 115.7 & 96.0 \\
1982 & 136.7 & 128.4 & 106.5 \\
1983 & 153.0 & 139.4 & 109.8 \\
1984 & 172.9 & 154.1 & 112.2 \\
1985 & 177.7 & 159.2 & 111.6 \\
1986 & 133.7 & 125.9 & 106.2 \\
1987 & 111.7 & 109.0 & 102.5 \\
1988 & 110.0 & 105.8 & 104.0 \\
1989 & 117.9 & 112.2 & 105.0 \\
1990 & 100.0 & 100.0 & 100.0 \\
1991 & 102.2 & 102.0 & 100.2 \\
1992 & 96.2 & 98.0 & 98.2 \\
1993 & 103.5 & 106.9 & 96.8 \\
1994 & 100.1 & 105.8 & 94.7 \\
\hline
\end{tabular}

\begin{tabular}{|l|c|c|}
\cline { 2 - 3 } \multicolumn{1}{c|}{} & \multicolumn{2}{c|}{ Trade Weights } \\
\cline { 2 - 3 } \multicolumn{1}{c|}{} & 1970 & 1994 \\
\hline Australia & 0.4 & 0.5 \\
Austria & 0.7 & 1.5 \\
Belgium & - & - \\
Canada & 1.0 & 0.7 \\
Denmark & 0.7 & 1.1 \\
Finland & 0.6 & 0.9 \\
France & 19.8 & 18.6 \\
Western Germany & 30.8 & 26.5 \\
Greece & 0.4 & 0.5 \\
Italy & 6.2 & 7.7 \\
Japan & 2.3 & 5.9 \\
Netherlands & 15.2 & 8.5 \\
Norway & 0.7 & 0.6 \\
Portugal & 0.3 & 0.9 \\
Spain & 0.8 & 3.4 \\
Sweden & 2.2 & 2.2 \\
United Kingdom & 6.8 & 10.4 \\
United States & 11.1 & 10.2 \\
\hline
\end{tabular}


Trade-Weighted Output per Employed Person and Unit Labour Costs in Total Manufacturing, 1979-94 (Indexes: 1990=100)

Competitors: Australia, Austria, Belgium, Denmark, Finland, France, Germany, Greece, Italy, Japan, Netherlands, Norway, Portugal, Spain, Sweden, United Kingdom, United States

\begin{tabular}{|c|c|c|c|}
\hline \multirow{2}{*}{ Year } & \multicolumn{3}{|c|}{ Output per Employed Person } \\
& \multicolumn{3}{|c|}{ U.S. Dollar Basis } \\
\cline { 2 - 4 } & Own & Competitors' & Ratio \\
\hline 1979 & 85.3 & 72.2 & 118.0 \\
1980 & 81.7 & 71.5 & 114.3 \\
1981 & 84.7 & 74.4 & 113.9 \\
1982 & 80.0 & 76.0 & 105.3 \\
1983 & 86.9 & 81.0 & 107.2 \\
1984 & 95.2 & 85.2 & 111.8 \\
1985 & 98.0 & 88.5 & 110.8 \\
1986 & 96.4 & 88.9 & 108.5 \\
1987 & 98.1 & 94.4 & 103.9 \\
1988 & 98.8 & 98.7 & 100.0 \\
1989 & 98.5 & 99.0 & 99.4 \\
1990 & 100.0 & 100.0 & 100.0 \\
1991 & 100.0 & 100.0 & 99.9 \\
1992 & 105.0 & 102.6 & 102.3 \\
1993 & 111.8 & 105.0 & 106.5 \\
1994 & 118.0 & 109.9 & 107.4 \\
\hline
\end{tabular}

\begin{tabular}{|c|r|r|r|}
\hline \multirow{2}{*}{ Year } & \multicolumn{3}{|c|}{ Unit Labour Costs } \\
& \multicolumn{3}{|c|}{ National Currency Basis } \\
\cline { 2 - 4 } & $\begin{array}{c}\text { Own } \\
\text { Index }\end{array}$ & $\begin{array}{c}\text { Competitors' } \\
\text { Index }\end{array}$ & Ratio \\
\hline 1979 & 58.3 & 72.9 & 79.9 \\
1980 & 67.0 & 81.5 & 82.2 \\
1981 & 73.6 & 86.2 & 85.4 \\
1982 & 85.3 & 91.2 & 93.5 \\
1983 & 84.3 & 90.5 & 93.1 \\
1984 & 81.4 & 90.7 & 89.8 \\
1985 & 83.2 & 92.1 & 90.4 \\
1986 & 87.8 & 95.5 & 91.9 \\
1987 & 89.6 & 93.2 & 96.1 \\
1988 & 93.2 & 93.1 & 100.1 \\
1989 & 96.3 & 96.2 & 100.1 \\
1990 & 100.0 & 100.0 & 100.0 \\
1991 & 105.9 & 104.6 & 101.3 \\
1992 & 105.0 & 107.6 & 97.6 \\
1993 & 101.6 & 108.6 & 93.6 \\
1994 & 99.3 & 107.3 & 92.6 \\
\hline
\end{tabular}

\begin{tabular}{|c|r|c|c|}
\hline \multirow{2}{*}{ Year } & \multicolumn{3}{|c|}{ Unit Labour Costs } \\
& \multicolumn{3}{|c|}{ U.S. Dollar Basis } \\
\cline { 2 - 4 } & Own & Competitors' & Ratio \\
\hline 1979 & 58.0 & 72.6 & 79.9 \\
1980 & 66.9 & 81.2 & 82.4 \\
1981 & 71.6 & 83.3 & 86.0 \\
1982 & 80.6 & 86.2 & 93.5 \\
1983 & 79.8 & 84.7 & 94.3 \\
1984 & 73.3 & 83.9 & 87.4 \\
1985 & 71.1 & 84.6 & 84.1 \\
1986 & 73.7 & 91.1 & 80.9 \\
1987 & 78.9 & 91.8 & 85.9 \\
1988 & 88.4 & 93.3 & 94.7 \\
1989 & 94.9 & 94.9 & 100.0 \\
1990 & 100.0 & 100.0 & 100.0 \\
1991 & 107.9 & 104.8 & 102.9 \\
1992 & 101.4 & 108.9 & 93.1 \\
1993 & 91.9 & 109.8 & 83.7 \\
1994 & 84.8 & 109.4 & 77.6 \\
\hline
\end{tabular}

\begin{tabular}{|c|c|c|r|}
\hline \multirow{2}{*}{ Year } & \multicolumn{3}{|c|}{$\begin{array}{c}\text { Exchange } \\
\text { Rates }\end{array}$} \\
\cline { 2 - 4 } & Own & $\begin{array}{c}\text { Competitors' } \\
\text { Index }\end{array}$ & Ratio \\
\hline 1979 & 100.4 & 100.9 & 99.5 \\
1980 & 100.2 & 101.6 & 98.7 \\
1981 & 102.8 & 104.9 & 98.0 \\
1982 & 105.7 & 108.7 & 97.3 \\
1983 & 105.6 & 109.9 & 96.1 \\
1984 & 111.0 & 112.1 & 99.0 \\
1985 & 117.0 & 113.7 & 103.0 \\
1986 & 119.1 & 106.0 & 112.4 \\
1987 & 113.6 & 101.6 & 111.8 \\
1988 & 105.5 & 100.0 & 105.5 \\
1989 & 101.5 & 101.7 & 99.8 \\
1990 & 100.0 & 100.0 & 100.0 \\
1991 & 98.2 & 99.8 & 98.4 \\
1992 & 103.6 & 98.9 & 104.7 \\
1993 & 110.6 & 99.8 & 110.8 \\
1994 & 117.0 & 99.3 & 117.9 \\
\hline
\end{tabular}

\begin{tabular}{|l|c|c|}
\cline { 2 - 3 } \multicolumn{1}{c|}{} & \multicolumn{2}{c|}{ Trade Weights } \\
\cline { 2 - 3 } \multicolumn{1}{c|}{} & 1970 & 1994 \\
\hline Australia & 1.1 & 0.4 \\
Austria & 0.4 & 0.2 \\
Belgium & 0.6 & 0.5 \\
Canada & - & - \\
Denmark & 0.2 & 0.2 \\
Finland & 0.2 & 0.2 \\
France & 1.7 & 1.5 \\
Western Germany & 3.5 & 2.6 \\
Greece & 0.1 & 0.0 \\
Italy & 1.4 & 1.5 \\
Japan & 5.4 & 6.8 \\
Netherlands & 0.7 & 0.5 \\
Norway & 0.1 & 0.2 \\
Portugal & 0.1 & 0.1 \\
Spain & 0.4 & 0.3 \\
Sweden & 0.9 & 0.5 \\
United Kingdom & 7.2 & 2.1 \\
United States & 76.2 & 82.4 \\
\hline
\end{tabular}


Trade-Weighted Output per Employed Person and Unit Labour Costs in Total Manufacturing, 1979-94 (Indexes: 1990=100)

Competitors: Australia, Austria, Belgium, Canada, Finland, France, Germany, Greece, Italy, Japan, Netherlands, Norway, Portugal, Spain, Sweden, United Kingdom, United States

\begin{tabular}{|c|r|c|c|}
\hline \multirow{2}{*}{ Year } & \multicolumn{3}{|c|}{ Output per Employed Person } \\
\cline { 2 - 4 } & $\begin{array}{c}\text { Own } \\
\text { Index }\end{array}$ & $\begin{array}{c}\text { Competitors' } \\
\text { Index }\end{array}$ & Ratio \\
\hline 1979 & 89.5 & 75.4 & 118.7 \\
1980 & 95.4 & 74.7 & 127.8 \\
1981 & 95.8 & 76.2 & 125.8 \\
1982 & 97.8 & 78.3 & 124.9 \\
1983 & 104.1 & 82.9 & 125.6 \\
1984 & 103.7 & 86.8 & 119.5 \\
1985 & 101.2 & 89.3 & 113.4 \\
1986 & 97.8 & 90.3 & 108.3 \\
1987 & 95.1 & 91.8 & 103.6 \\
1988 & 98.8 & 95.4 & 103.5 \\
1989 & 101.1 & 98.2 & 102.9 \\
1990 & 100.0 & 100.0 & 100.0 \\
1991 & 102.3 & 101.4 & 100.9 \\
1992 & 104.8 & 103.7 & 101.1 \\
1993 & 110.4 & 105.4 & 104.8 \\
1994 & 112.2 & 112.6 & 99.6 \\
\hline
\end{tabular}

\begin{tabular}{|c|r|r|r|}
\hline \multirow{2}{*}{ Year } & \multicolumn{3}{|c|}{ Unit Labour Costs } \\
& \multicolumn{3}{|c|}{ National Currency Basis } \\
\cline { 2 - 4 } & $\begin{array}{r}\text { Own } \\
\text { Index }\end{array}$ & $\begin{array}{c}\text { Competitors' } \\
\text { Index }\end{array}$ & Ratio \\
\hline 1979 & 54.8 & 63.4 & 86.5 \\
1980 & 57.4 & 70.7 & 81.2 \\
1981 & 62.2 & 76.0 & 81.8 \\
1982 & 67.4 & 80.0 & 84.3 \\
1983 & 68.7 & 80.9 & 84.9 \\
1984 & 73.0 & 82.9 & 88.0 \\
1985 & 77.4 & 85.7 & 90.3 \\
1986 & 83.9 & 89.4 & 93.9 \\
1987 & 92.2 & 92.6 & 99.7 \\
1988 & 92.0 & 93.4 & 98.5 \\
1989 & 94.1 & 95.5 & 98.5 \\
1990 & 100.0 & 100.0 & 100.0 \\
1991 & 102.0 & 105.1 & 97.0 \\
1992 & 103.7 & 108.3 & 95.7 \\
1993 & 99.8 & 109.7 & 91.0 \\
1994 & 101.3 & 107.1 & 94.6 \\
\hline
\end{tabular}

\begin{tabular}{|c|c|c|c|}
\hline \multirow[t]{2}{*}{ Year } & \multicolumn{3}{|c|}{$\begin{array}{l}\text { Unit Labour Costs } \\
\text { U.S. Dollar Basis }\end{array}$} \\
\hline & $\begin{array}{l}\text { Own } \\
\text { Index }\end{array}$ & $\begin{array}{l}\text { Competitors' } \\
\text { Index }\end{array}$ & Ratio \\
\hline 1979 & 64.5 & 67.9 & 95.0 \\
\hline 1980 & 63.0 & 77.2 & 81.6 \\
\hline 1981 & 54.0 & 69.7 & 77.5 \\
\hline 1982 & 50.1 & 65.1 & 76.9 \\
\hline 1983 & 46.5 & 60.1 & 77.4 \\
\hline 1984 & 43.6 & 56.7 & 77.0 \\
\hline 1985 & 45.2 & 56.8 & 79.7 \\
\hline 1986 & 64.2 & 72.2 & 88.9 \\
\hline 1987 & 83.5 & 85.4 & 97.7 \\
\hline 1988 & 84.6 & 89.3 & 94.7 \\
\hline 1989 & 79.6 & 86.1 & 92.5 \\
\hline 1990 & 100.0 & 100.0 & 100.0 \\
\hline 1991 & 98.6 & 103.2 & 95.5 \\
\hline 1992 & 106.3 & 110.4 & 96.3 \\
\hline 1993 & 95.3 & 102.0 & 93.3 \\
\hline 1994 & 98.6 & 101.6 & 97.1 \\
\hline
\end{tabular}

\begin{tabular}{|c|r|c|r|}
\hline \multirow{2}{*}{ Year } & \multicolumn{3}{|c|}{ Exchange } \\
& \multicolumn{3}{|c|}{ Rates } \\
\cline { 2 - 4 } & Own & $\begin{array}{c}\text { Competitors' } \\
\text { Index }\end{array}$ & Ratio \\
\hline 1979 & 85.0 & 93.9 & 90.6 \\
1980 & 91.1 & 92.8 & 98.1 \\
1981 & 115.1 & 111.3 & 103.4 \\
1982 & 134.6 & 125.0 & 107.7 \\
1983 & 147.8 & 136.9 & 107.9 \\
1984 & 167.3 & 150.1 & 111.5 \\
1985 & 171.2 & 155.5 & 110.1 \\
1986 & 130.7 & 124.6 & 104.9 \\
1987 & 110.5 & 108.5 & 101.9 \\
1988 & 108.8 & 104.9 & 103.7 \\
1989 & 118.1 & 111.3 & 106.1 \\
1990 & 100.0 & 100.0 & 100.0 \\
1991 & 103.4 & 101.9 & 101.5 \\
1992 & 97.5 & 98.3 & 99.2 \\
1993 & 104.8 & 110.1 & 95.1 \\
1994 & 102.8 & 108.7 & 94.6 \\
\hline
\end{tabular}

\begin{tabular}{|l|c|c|}
\cline { 2 - 3 } \multicolumn{1}{c|}{} & \multicolumn{2}{c|}{ Trade Weights } \\
\cline { 2 - 3 } \multicolumn{1}{c|}{} & 1970 & 1994 \\
\hline Australia & 0.3 & 0.5 \\
Austria & 2.0 & 1.6 \\
Belgium & 3.1 & 3.6 \\
Canada & 0.8 & 0.5 \\
Denmark & - & - \\
Finland & 2.9 & 3.2 \\
France & 5.3 & 7.9 \\
Western Germany & 21.5 & 27.1 \\
Greece & 0.2 & 0.5 \\
Italy & 4.4 & 6.3 \\
Japan & 2.7 & 6.4 \\
Netherlands & 3.6 & 5.4 \\
Norway & 5.5 & 4.5 \\
Portugal & 0.8 & 1.1 \\
Spain & 0.8 & 2.0 \\
Sweden & 19.3 & 11.7 \\
United Kingdom & 19.1 & 10.1 \\
United States & 7.6 & 7.8 \\
\hline
\end{tabular}


Trade-Weighted Output per Employed Person and Unit Labour Costs in Total Manufacturing, 1979-94 (Indexes: 1990=100)

Competitors: Australia, Austria, Belgium, Canada, Denmark, France, Germany, Greece, Italy, Japan, Netherlands, Norway, Portugal, Spain, Sweden, United Kingdom, United States

\begin{tabular}{|c|r|c|r|}
\hline \multirow{2}{*}{ Year } & \multicolumn{3}{|c|}{ Output per Employed Person } \\
\cline { 2 - 4 } & Own & Competitors' & Ratio \\
\hline & Index & Index & 79.1 \\
& 60.4 & 76.4 & 82.3 \\
1980 & 62.5 & 75.9 & 84.5 \\
1981 & 65.3 & 77.3 & 84.4 \\
1982 & 67.0 & 79.4 & 83.4 \\
1983 & 70.1 & 84.0 & 84.0 \\
1984 & 73.9 & 88.0 & 86.0 \\
1985 & 77.5 & 90.1 & 90.0 \\
1986 & 81.8 & 90.9 & 95.8 \\
1987 & 88.2 & 92.1 & 98.5 \\
1988 & 94.2 & 95.7 & 101.0 \\
1989 & 99.2 & 98.3 & 100.0 \\
1990 & 100.0 & 100.0 & 94.6 \\
1991 & 96.2 & 101.7 & 104.3 \\
1992 & 108.4 & 104.0 & 115.6 \\
1993 & 122.3 & 105.8 & 120.9 \\
1994 & 136.0 & 112.5 & \\
\hline
\end{tabular}

\begin{tabular}{|c|r|c|c|}
\hline \multirow{2}{*}{ Year } & \multicolumn{3}{|c|}{ Unit Labour Costs } \\
& \multicolumn{3}{|c|}{ National Currency Basis } \\
\cline { 2 - 4 } & Own & Competitors' & Ratio \\
\hline 1979 & 58.0 & 61.8 & 93.8 \\
1980 & 63.1 & 69.2 & 91.1 \\
1981 & 69.0 & 74.5 & 92.6 \\
1982 & 74.3 & 78.8 & 94.3 \\
1983 & 77.4 & 80.1 & 96.6 \\
1984 & 80.6 & 81.8 & 98.5 \\
1985 & 84.2 & 85.0 & 99.1 \\
1986 & 86.1 & 88.8 & 97.0 \\
1987 & 86.4 & 92.1 & 93.8 \\
1988 & 88.7 & 93.0 & 95.3 \\
1989 & 92.4 & 95.4 & 96.9 \\
1990 & 100.0 & 100.0 & 100.0 \\
1991 & 105.9 & 105.2 & 100.7 \\
1992 & 97.4 & 108.3 & 90.0 \\
1993 & 91.3 & 109.2 & 83.6 \\
1994 & 86.8 & 107.0 & 81.2 \\
\hline
\end{tabular}

\begin{tabular}{|c|r|c|c|}
\hline \multirow{2}{*}{ Year } & \multicolumn{3}{|c|}{ Unit Labour Costs } \\
& \multicolumn{3}{|c|}{ U.S. Dollar Basis } \\
\cline { 2 - 4 } & Own & Competitors' & Ratio \\
\hline 1979 & 56.9 & 67.9 & 83.8 \\
1980 & 64.6 & 76.5 & 84.5 \\
1981 & 61.1 & 69.8 & 87.6 \\
1982 & 58.9 & 64.4 & 91.5 \\
1983 & 53.1 & 59.3 & 89.6 \\
1984 & 51.2 & 55.6 & 92.1 \\
1985 & 51.9 & 55.7 & 93.3 \\
1986 & 64.9 & 71.7 & 90.5 \\
1987 & 75.2 & 85.2 & 88.2 \\
1988 & 81.1 & 89.5 & 90.6 \\
1989 & 82.4 & 86.4 & 95.4 \\
1990 & 100.0 & 100.0 & 100.0 \\
1991 & 100.1 & 103.4 & 96.8 \\
1992 & 83.2 & 110.8 & 75.1 \\
1993 & 61.1 & 102.1 & 59.8 \\
1994 & 63.6 & 102.4 & 62.1 \\
\hline
\end{tabular}

\begin{tabular}{|c|r|c|c|}
\hline \multirow{2}{*}{ Year } & \multicolumn{3}{|c|}{$\begin{array}{c}\text { Exchange } \\
\text { Rates }\end{array}$} \\
\cline { 2 - 4 } & Own & $\begin{array}{c}\text { Competitors' } \\
\text { Index }\end{array}$ & Ratio \\
\hline 1979 & 101.9 & 91.6 & 111.2 \\
1980 & 97.6 & 92.0 & 106.0 \\
1981 & 112.9 & 109.0 & 103.6 \\
1982 & 126.1 & 124.6 & 101.2 \\
1983 & 145.7 & 137.2 & 106.1 \\
1984 & 157.2 & 150.5 & 104.5 \\
1985 & 162.1 & 156.5 & 103.6 \\
1986 & 132.6 & 124.5 & 106.5 \\
1987 & 115.0 & 108.2 & 106.2 \\
1988 & 109.4 & 104.3 & 104.9 \\
1989 & 112.2 & 110.9 & 101.2 \\
1990 & 100.0 & 100.0 & 100.0 \\
1991 & 105.8 & 101.7 & 104.0 \\
1992 & 117.2 & 97.9 & 119.7 \\
1993 & 149.4 & 109.4 & 136.6 \\
1994 & 136.6 & 107.8 & 126.8 \\
\hline
\end{tabular}

\begin{tabular}{|l|c|c|}
\cline { 2 - 3 } \multicolumn{1}{c|}{} & \multicolumn{2}{c|}{ Trade Weights } \\
\cline { 2 - 3 } \multicolumn{1}{c|}{} & 1970 & 1994 \\
\hline Australia & 0.5 & 0.9 \\
Austria & 2.0 & 1.7 \\
Belgium & 2.8 & 3.5 \\
Canada & 0.7 & 0.8 \\
Denmark & 4.1 & 4.4 \\
Finland & - & - \\
France & 5.6 & 7.1 \\
Western Germany & 22.3 & 21.9 \\
Greece & 0.2 & 0.3 \\
Italy & 3.5 & 5.9 \\
Japan & 3.5 & 7.9 \\
Netherlands & 3.2 & 4.6 \\
Norway & 3.1 & 3.1 \\
Portugal & 0.9 & 0.9 \\
Spain & 0.7 & 2.1 \\
Sweden & 21.2 & 14.5 \\
United Kingdom & 18.6 & 10.7 \\
United States & 6.9 & 9.7 \\
\hline
\end{tabular}


Trade-Weighted Output per Employed Person and Unit Labour Costs in Total Manufacturing, 1979-94 (Indexes: 1990=100)

Competitors: Australia, Austria, Belgium, Canada, Denmark, Finland, Germany, Greece, Italy, Japan, Netherlands, Norway, Portugal, Spain, Sweden, United Kingdom, United States

\begin{tabular}{|c|c|c|c|}
\hline \multirow{2}{*}{ Year } & \multicolumn{3}{|c|}{ Output per Employed Person } \\
& \multicolumn{3}{|c|}{ U.S. Dollar Basis } \\
\cline { 2 - 4 } & Own & Competitors' & Ratio \\
\hline 1979 & 76.0 & 74.0 & 102.6 \\
1980 & 76.4 & 73.9 & 103.3 \\
1981 & 78.4 & 75.9 & 103.3 \\
1982 & 80.3 & 77.7 & 103.4 \\
1983 & 82.2 & 82.2 & 100.1 \\
1984 & 83.1 & 86.4 & 96.2 \\
1985 & 85.1 & 89.2 & 95.4 \\
1986 & 86.5 & 90.4 & 95.7 \\
1987 & 87.9 & 91.9 & 95.6 \\
1988 & 94.5 & 95.9 & 98.5 \\
1989 & 98.8 & 98.4 & 100.4 \\
1990 & 100.0 & 100.0 & 100.0 \\
1991 & 99.7 & 101.4 & 98.3 \\
1992 & 100.9 & 103.0 & 97.9 \\
1993 & 101.7 & 103.5 & 98.3 \\
1994 & 109.4 & 109.8 & 99.6 \\
\hline
\end{tabular}

\begin{tabular}{|c|c|c|c|}
\hline \multirow{2}{*}{ Year } & \multicolumn{3}{|c|}{ Unit Labour Costs } \\
& \multicolumn{3}{|c|}{ National Currency Basis } \\
\cline { 2 - 4 } & Own & Competitors' & Ratio \\
\hline 1979 & 54.7 & 66.6 & 82.1 \\
1980 & 62.7 & 73.2 & 85.7 \\
1981 & 70.0 & 77.7 & 90.1 \\
1982 & 77.6 & 82.1 & 94.5 \\
1983 & 83.0 & 82.9 & 100.2 \\
1984 & 89.3 & 84.3 & 105.9 \\
1985 & 94.1 & 86.9 & 108.3 \\
1986 & 96.6 & 89.8 & 107.5 \\
1987 & 99.0 & 93.0 & 106.5 \\
1988 & 96.1 & 93.3 & 103.0 \\
1989 & 96.5 & 95.4 & 101.1 \\
1990 & 100.0 & 100.0 & 100.0 \\
1991 & 105.3 & 105.1 & 100.2 \\
1992 & 107.7 & 109.4 & 98.4 \\
1993 & 109.6 & 112.1 & 97.8 \\
1994 & 106.5 & 109.7 & 97.1 \\
\hline
\end{tabular}

\begin{tabular}{|c|r|c|r|}
\hline \multirow{2}{*}{ Year } & \multicolumn{3}{|c|}{ Unit Labour Costs } \\
& \multicolumn{3}{|c|}{ U.S. Dollar Basis } \\
\cline { 2 - 4 } & Own & Competitors' & \multicolumn{1}{c|}{ Ratio } \\
\hline 1979 & 70.0 & 69.9 & 100.1 \\
1980 & 80.8 & 77.5 & 104.3 \\
1981 & 70.2 & 68.6 & 102.3 \\
1982 & 64.3 & 64.9 & 99.2 \\
1983 & 59.3 & 60.6 & 97.9 \\
1984 & 55.6 & 56.3 & 98.7 \\
1985 & 57.0 & 55.9 & 102.1 \\
1986 & 75.9 & 71.5 & 106.1 \\
1987 & 89.7 & 85.2 & 105.2 \\
1988 & 87.9 & 88.2 & 99.6 \\
1989 & 82.3 & 85.2 & 96.7 \\
1990 & 100.0 & 100.0 & 100.0 \\
1991 & 101.7 & 103.3 & 98.4 \\
1992 & 110.8 & 111.4 & 99.4 \\
1993 & 105.4 & 104.2 & 101.2 \\
1994 & 104.5 & 102.9 & 101.5 \\
\hline
\end{tabular}

\begin{tabular}{|c|r|c|r|}
\hline \multirow{2}{*}{ Year } & \multicolumn{3}{|c|}{$\begin{array}{c}\text { Exchange } \\
\text { Rates }\end{array}$} \\
\cline { 2 - 4 } & $\begin{array}{c}\text { Own } \\
\text { Index }\end{array}$ & $\begin{array}{c}\text { Competitors' } \\
\text { Index }\end{array}$ & Ratio \\
\hline 1979 & 78.1 & 95.8 & 81.6 \\
1980 & 77.6 & 95.4 & 81.3 \\
1981 & 99.8 & 115.4 & 86.5 \\
1982 & 120.7 & 129.0 & 93.5 \\
1983 & 140.0 & 139.5 & 100.4 \\
1984 & 160.5 & 154.4 & 104.0 \\
1985 & 165.0 & 160.8 & 102.6 \\
1986 & 127.2 & 126.6 & 100.4 \\
1987 & 110.4 & 109.3 & 101.0 \\
1988 & 109.4 & 106.1 & 103.1 \\
1989 & 117.2 & 112.4 & 104.2 \\
1990 & 100.0 & 100.0 & 100.0 \\
1991 & 103.6 & 101.8 & 101.8 \\
1992 & 97.2 & 98.4 & 98.8 \\
1993 & 104.0 & 109.5 & 95.0 \\
1994 & 102.0 & 109.1 & 93.5 \\
\hline
\end{tabular}

\begin{tabular}{|l|c|r|}
\cline { 2 - 3 } \multicolumn{1}{c|}{} & \multicolumn{2}{c|}{ Trade Weights } \\
\cline { 2 - 3 } \multicolumn{1}{c|}{} & 1970 & 1994 \\
\hline Australia & 0.7 & 0.4 \\
Austria & 0.7 & 1.4 \\
Belgium & 16.1 & 11.0 \\
Canada & 1.0 & 0.6 \\
Denmark & 0.7 & 1.1 \\
Finland & 0.8 & 0.9 \\
France & - & - \\
Western Germany & 32.8 & 27.4 \\
Greece & 0.3 & 0.4 \\
Italy & 14.0 & 13.6 \\
Japan & 1.8 & 4.9 \\
Netherlands & 7.4 & 6.5 \\
Norway & 0.7 & 0.6 \\
Portugal & 0.4 & 1.5 \\
Spain & 1.5 & 7.5 \\
Sweden & 2.7 & 1.9 \\
United Kingdom & 6.8 & 11.0 \\
United States & 11.6 & 9.5 \\
\hline
\end{tabular}


Trade-Weighted Output per Employed Person and Unit Labour Costs in Total Manufacturing, 1979-94 (Indexes: 1990=100)

Competitors: Australia, Austria, Belgium, Canada, Denmark, Finland, France, Greece, Italy, Japan, Netherlands, Norway, Portugal, Spain, Sweden, United Kingdom, United States

\begin{tabular}{|c|r|c|c|}
\hline \multirow{2}{*}{ Year } & \multicolumn{3}{|c|}{ Output per Employed Person } \\
\cline { 2 - 4 } & Own & Competitors' & Ratio \\
\cline { 2 - 4 } & Index & 70.7 & 123.2 \\
1979 & 87.1 & 71.6 & 118.0 \\
1980 & 84.5 & 73.8 & 115.3 \\
1981 & 85.1 & 76.2 & 111.1 \\
1982 & 84.7 & 80.6 & 110.4 \\
1983 & 89.0 & 84.5 & 109.0 \\
1984 & 92.0 & 87.1 & 108.2 \\
1985 & 94.2 & 88.3 & 106.6 \\
1986 & 94.2 & 90.6 & 102.0 \\
1987 & 92.4 & 95.3 & 100.2 \\
1988 & 95.5 & 98.5 & 98.9 \\
1989 & 97.4 & 100.0 & 100.0 \\
1990 & 100.0 & 100.9 & 101.3 \\
1991 & 102.3 & 103.3 & 98.0 \\
1992 & 101.2 & 104.9 & 94.4 \\
1993 & 99.0 & 111.2 & 96.0 \\
1994 & 106.7 & &
\end{tabular}

\begin{tabular}{|c|c|c|c|}
\hline \multirow{2}{*}{ Year } & \multicolumn{3}{|c|}{ Unit Labour Costs } \\
& \multicolumn{3}{|c|}{ National Currency Basis } \\
\cline { 2 - 4 } & Own & Competitors' & Ratio \\
\hline 1979 & 69.6 & 66.7 & 104.3 \\
1980 & 76.8 & 73.1 & 105.1 \\
1981 & 80.1 & 78.4 & 102.2 \\
1982 & 84.8 & 83.1 & 102.1 \\
1983 & 84.4 & 84.6 & 99.8 \\
1984 & 85.5 & 86.3 & 99.1 \\
1985 & 87.0 & 89.2 & 97.5 \\
1986 & 90.1 & 92.3 & 97.7 \\
1987 & 95.8 & 94.4 & 101.5 \\
1988 & 96.1 & 93.8 & 102.4 \\
1989 & 97.5 & 95.4 & 102.2 \\
1990 & 100.0 & 100.0 & 100.0 \\
1991 & 103.2 & 105.5 & 97.8 \\
1992 & 110.4 & 108.5 & 101.7 \\
1993 & 115.2 & 110.3 & 104.4 \\
1994 & 111.6 & 108.0 & 103.3 \\
\hline
\end{tabular}

\begin{tabular}{|c|r|c|r|}
\hline \multirow{2}{*}{ Year } & \multicolumn{3}{|c|}{ Unit Labour Costs } \\
& \multicolumn{3}{|c|}{ U.S. Dollar Basis } \\
\cline { 2 - 4 } & Own & Competitors' & Ratio \\
\hline 1979 & Index & 72.9 & 84.1 \\
1980 & 68.3 & 80.6 & 84.8 \\
1981 & 57.3 & 72.0 & 79.6 \\
1982 & 56.5 & 67.7 & 83.4 \\
1983 & 53.4 & 62.9 & 84.9 \\
1984 & 48.5 & 58.8 & 82.5 \\
1985 & 47.7 & 59.0 & 80.9 \\
1986 & 67.1 & 75.0 & 89.3 \\
1987 & 86.2 & 87.4 & 98.6 \\
1988 & 88.4 & 89.8 & 98.4 \\
1989 & 83.8 & 86.2 & 97.1 \\
1990 & 100.0 & 100.0 & 100.0 \\
1991 & 100.5 & 103.8 & 96.8 \\
1992 & 114.2 & 110.6 & 103.2 \\
1993 & 112.6 & 103.5 & 108.7 \\
1994 & 111.1 & 103.1 & 107.8 \\
\hline
\end{tabular}

\begin{tabular}{|c|c|c|c|}
\hline \multirow{2}{*}{ Year } & \multicolumn{3}{|c|}{$\begin{array}{c}\text { Exchange } \\
\text { Rates }\end{array}$} \\
\cline { 2 - 4 } & Own & $\begin{array}{c}\text { Competitors' } \\
\text { Index }\end{array}$ & Ratio \\
\hline 1979 & 113.4 & 91.0 & 124.6 \\
1980 & 112.5 & 91.3 & 123.3 \\
1981 & 139.9 & 109.5 & 127.7 \\
1982 & 150.2 & 124.2 & 120.9 \\
1983 & 158.0 & 136.4 & 115.8 \\
1984 & 176.1 & 150.6 & 117.0 \\
1985 & 182.2 & 155.9 & 116.9 \\
1986 & 134.4 & 123.8 & 108.5 \\
1987 & 111.2 & 108.2 & 102.8 \\
1988 & 108.7 & 104.9 & 103.6 \\
1989 & 116.4 & 111.2 & 104.6 \\
1990 & 100.0 & 100.0 & 100.0 \\
1991 & 102.7 & 101.7 & 101.0 \\
1992 & 96.7 & 98.3 & 98.3 \\
1993 & 102.3 & 108.9 & 94.0 \\
1994 & 100.4 & 107.9 & 93.1 \\
\hline
\end{tabular}

\begin{tabular}{|l|c|c|}
\cline { 2 - 3 } \multicolumn{1}{c|}{} & \multicolumn{2}{c|}{ Trade Weights } \\
\cline { 2 - 3 } \multicolumn{1}{c|}{} & 1970 & 1994 \\
\hline Australia & 0.5 & 0.5 \\
Austria & 3.7 & 6.8 \\
Belgium & 13.8 & 9.3 \\
Canada & 1.5 & 0.7 \\
Denmark & 1.7 & 2.8 \\
Finland & 1.3 & 1.6 \\
France & 18.1 & 15.9 \\
Western Germany & - & - \\
Greece & 0.4 & 0.7 \\
Italy & 14.1 & 13.6 \\
Japan & 3.5 & 8.2 \\
Netherlands & 14.9 & 10.7 \\
Norway & 2.0 & 1.0 \\
Portugal & 0.4 & 1.3 \\
Spain & 1.2 & 3.8 \\
Sweden & 3.9 & 3.3 \\
United Kingdom & 6.4 & 9.9 \\
United States & 12.6 & 9.9 \\
\hline
\end{tabular}


Trade-Weighted Output per Employed Person and Unit Labour Costs in Total Manufacturing, 1979-94 (Indexes: 1990=100)

Competitors: Australia, Austria, Belgium, Canada, Denmark, Finland, France, Germany, Italy, Japan, Netherlands, Norway, Portugal, Spain, Sweden, United Kingdom, United States

\begin{tabular}{|c|c|c|c|}
\hline \multirow{2}{*}{ Year } & \multicolumn{3}{|c|}{ Output per Employed Person } \\
& \multicolumn{3}{|c|}{ U.S. Dollar Basis } \\
\cline { 2 - 4 } & $\begin{array}{c}\text { Own } \\
\text { Index }\end{array}$ & $\begin{array}{c}\text { Competitors' } \\
\text { Index }\end{array}$ & Ratio \\
\hline 1979 & 90.7 & 74.5 & 121.8 \\
1980 & 89.9 & 74.8 & 120.2 \\
1981 & 89.2 & 76.4 & 116.8 \\
1982 & 90.6 & 77.8 & 116.5 \\
1983 & 91.7 & 81.4 & 112.6 \\
1984 & 92.8 & 85.4 & 108.7 \\
1985 & 97.0 & 88.5 & 109.6 \\
1986 & 99.0 & 89.9 & 110.1 \\
1987 & 95.4 & 91.3 & 104.5 \\
1988 & 99.3 & 95.6 & 103.9 \\
1989 & 101.4 & 98.3 & 103.1 \\
1990 & 100.0 & 100.0 & 100.0 \\
1991 & 105.1 & 101.2 & 103.8 \\
1992 & 106.6 & 102.8 & 103.7 \\
1993 & 103.2 & 103.5 & 99.7 \\
1994 & 105.4 & 110.3 & 95.5 \\
\hline
\end{tabular}

\begin{tabular}{|c|c|c|c|}
\hline \multirow{2}{*}{ Year } & \multicolumn{3}{|c|}{ Unit Labour Costs } \\
& \multicolumn{3}{|c|}{ National Currency Basis } \\
\cline { 2 - 4 } & Own & Competitors' & Ratio \\
\hline 1979 & 13.3 & 64.8 & 20.6 \\
1980 & 16.6 & 71.5 & 23.3 \\
1981 & 20.6 & 77.8 & 26.5 \\
1982 & 27.0 & 82.4 & 32.7 \\
1983 & 32.1 & 84.3 & 38.0 \\
1984 & 39.9 & 86.0 & 46.5 \\
1985 & 45.7 & 88.1 & 51.8 \\
1986 & 50.7 & 91.0 & 55.7 \\
1987 & 58.9 & 94.1 & 62.6 \\
1988 & 67.5 & 93.9 & 71.9 \\
1989 & 81.4 & 95.7 & 85.1 \\
1990 & 100.0 & 100.0 & 100.0 \\
1991 & 114.3 & 105.1 & 108.8 \\
1992 & 131.4 & 109.1 & 120.5 \\
1993 & 155.9 & 111.4 & 139.9 \\
1994 & 174.0 & 108.7 & 160.1 \\
\hline
\end{tabular}

\begin{tabular}{|c|r|c|r|}
\hline \multirow{2}{*}{ Year } & \multicolumn{3}{|c|}{ Unit Labour Costs } \\
& \multicolumn{3}{|c|}{ U.S. Dollar Basis } \\
\cline { 2 - 4 } & Own & Competitors' & \multicolumn{1}{c|}{ Ratio } \\
\hline 1979 & 57.1 & 66.5 & 85.9 \\
1980 & 61.9 & 74.9 & 82.6 \\
1981 & 59.0 & 66.9 & 88.1 \\
1982 & 64.1 & 64.1 & 99.9 \\
1983 & 57.8 & 60.0 & 96.2 \\
1984 & 56.2 & 55.9 & 100.5 \\
1985 & 52.4 & 55.0 & 95.3 \\
1986 & 57.4 & 71.9 & 79.9 \\
1987 & 69.0 & 86.1 & 80.1 \\
1988 & 75.4 & 88.3 & 85.4 \\
1989 & 79.5 & 84.9 & 93.5 \\
1990 & 100.0 & 100.0 & 100.0 \\
1991 & 99.4 & 103.2 & 96.4 \\
1992 & 109.3 & 111.1 & 98.4 \\
1993 & 107.8 & 103.8 & 103.8 \\
1994 & 113.7 & 101.3 & 112.3 \\
\hline
\end{tabular}

\begin{tabular}{|c|c|c|c|}
\hline \multirow{2}{*}{ Year } & \multicolumn{3}{|c|}{$\begin{array}{c}\text { Exchange } \\
\text { Rates }\end{array}$} \\
\cline { 2 - 4 } & $\begin{array}{c}\text { Own } \\
\text { Index }\end{array}$ & $\begin{array}{c}\text { Competitors' } \\
\text { Index }\end{array}$ & Ratio \\
\hline 1979 & 23.4 & 98.1 & 23.8 \\
1980 & 26.9 & 96.8 & 27.8 \\
1981 & 35.0 & 118.0 & 29.6 \\
1982 & 42.1 & 130.7 & 32.2 \\
1983 & 55.6 & 142.4 & 39.0 \\
1984 & 71.1 & 157.0 & 45.3 \\
1985 & 87.1 & 163.8 & 53.2 \\
1986 & 88.3 & 127.4 & 69.3 \\
1987 & 85.4 & 109.4 & 78.1 \\
1988 & 89.5 & 106.6 & 83.9 \\
1989 & 102.5 & 113.0 & 90.7 \\
1990 & 100.0 & 100.0 & 100.0 \\
1991 & 115.0 & 101.9 & 112.8 \\
1992 & 120.3 & 98.3 & 122.3 \\
1993 & 144.6 & 109.6 & 132.0 \\
1994 & 153.0 & 110.2 & 138.9 \\
\hline
\end{tabular}

\begin{tabular}{|l|c|c|}
\cline { 2 - 3 } \multicolumn{1}{c|}{} & \multicolumn{2}{c|}{ Trade Weights } \\
\cline { 2 - 3 } \multicolumn{1}{c|}{} & 1970 & 1994 \\
\hline Australia & 0.8 & 0.3 \\
Austria & 1.9 & 1.6 \\
Belgium & 4.1 & 4.7 \\
Canada & 1.3 & 0.5 \\
Denmark & 0.9 & 1.6 \\
Finland & 1.1 & 1.1 \\
France & 9.0 & 10.7 \\
Western Germany & 22.5 & 22.9 \\
Greece & - & - \\
Italy & 11.7 & 20.9 \\
Japan & 18.6 & 4.9 \\
Netherlands & 3.9 & 7.7 \\
Norway & 3.7 & 0.7 \\
Portugal & 0.2 & 0.6 \\
Spain & 0.6 & 4.0 \\
Sweden & 1.6 & 2.2 \\
United Kingdom & 8.8 & 8.8 \\
United States & 9.2 & 7.0 \\
\hline
\end{tabular}


Trade-Weighted Output per Employed Person and Unit Labour Costs in Total Manufacturing, 1979-94 (Indexes: 1990=100)

Competitors: Australia, Austria, Belgium, Canada, Denmark, Finland, France, Germany, Greece, Japan, Netherlands, Norway, Portugal, Spain, Sweden, United Kingdom, United States

\begin{tabular}{|c|c|c|c|}
\hline \multirow{2}{*}{ Year } & \multicolumn{3}{|c|}{ Output per Employed Person } \\
\cline { 2 - 4 } & Own & Competitors' & Ratio \\
\hline 1979 & 65.5 & 77.0 & 85.0 \\
1980 & 67.9 & 76.3 & 89.0 \\
1981 & 69.0 & 78.1 & 88.4 \\
1982 & 70.0 & 79.8 & 87.7 \\
1983 & 73.5 & 84.0 & 87.5 \\
1984 & 80.2 & 86.9 & 92.3 \\
1985 & 84.0 & 89.4 & 93.9 \\
1986 & 86.7 & 90.4 & 95.9 \\
1987 & 91.1 & 91.3 & 99.7 \\
1988 & 95.8 & 95.7 & 100.1 \\
1989 & 98.6 & 98.4 & 100.3 \\
1990 & 100.0 & 100.0 & 100.0 \\
1991 & 101.3 & 101.3 & 100.0 \\
1992 & 105.2 & 102.4 & 102.8 \\
1993 & 107.2 & 102.7 & 104.4 \\
1994 & 113.2 & 109.4 & 103.5 \\
\hline
\end{tabular}

\begin{tabular}{|c|c|c|c|}
\hline \multirow{2}{*}{ Year } & \multicolumn{3}{|c|}{ Unit Labour Costs } \\
& \multicolumn{3}{|c|}{ National Currency Basis } \\
\cline { 2 - 4 } & Own & Competitors' & Ratio \\
\hline 1979 & 42.8 & 66.3 & 64.6 \\
1980 & 48.0 & 73.3 & 65.5 \\
1981 & 56.8 & 78.3 & 72.5 \\
1982 & 65.5 & 83.1 & 78.9 \\
1983 & 72.8 & 84.0 & 86.6 \\
1984 & 76.2 & 86.1 & 88.5 \\
1985 & 81.0 & 88.9 & 91.2 \\
1986 & 83.2 & 92.0 & 90.4 \\
1987 & 85.7 & 95.2 & 90.0 \\
1988 & 87.6 & 94.7 & 92.4 \\
1989 & 93.3 & 96.0 & 97.2 \\
1990 & 100.0 & 100.0 & 100.0 \\
1991 & 108.0 & 104.6 & 103.3 \\
1992 & 110.9 & 109.0 & 101.7 \\
1993 & 113.3 & 111.7 & 101.4 \\
1994 & 110.1 & 109.5 & 100.5 \\
\hline
\end{tabular}

\begin{tabular}{|c|r|c|c|}
\hline \multirow{2}{*}{ Year } & \multicolumn{3}{|c|}{ Unit Labour Costs } \\
& \multicolumn{3}{|c|}{ U.S. Dollar Basis } \\
\cline { 2 - 4 } & Own & Competitors' & Ratio \\
\hline 1979 & 61.7 & 69.1 & 89.4 \\
1980 & 67.2 & 77.8 & 86.4 \\
1981 & 59.8 & 69.2 & 86.5 \\
1982 & 58.0 & 65.7 & 88.3 \\
1983 & 57.4 & 61.0 & 94.1 \\
1984 & 52.0 & 57.2 & 90.8 \\
1985 & 50.8 & 57.3 & 88.7 \\
1986 & 66.9 & 73.2 & 91.4 \\
1987 & 79.3 & 87.1 & 91.0 \\
1988 & 80.6 & 89.3 & 90.3 \\
1989 & 81.4 & 85.2 & 95.5 \\
1990 & 100.0 & 100.0 & 100.0 \\
1991 & 104.3 & 102.6 & 101.7 \\
1992 & 107.8 & 111.6 & 96.5 \\
1993 & 86.3 & 107.1 & 80.5 \\
1994 & 81.8 & 106.6 & 76.7 \\
\hline
\end{tabular}

\begin{tabular}{|c|c|c|c|}
\hline \multirow{2}{*}{ Year } & \multicolumn{3}{|c|}{$\begin{array}{c}\text { Exchange } \\
\text { Rates }\end{array}$} \\
\cline { 2 - 4 } & $\begin{array}{c}\text { Own } \\
\text { Index }\end{array}$ & $\begin{array}{c}\text { Competitors' } \\
\text { Index }\end{array}$ & Ratio \\
\hline 1979 & 69.3 & 96.6 & 71.8 \\
1980 & 71.5 & 95.5 & 74.8 \\
1981 & 94.9 & 115.6 & 82.0 \\
1982 & 112.9 & 129.1 & 87.4 \\
1983 & 126.8 & 140.7 & 90.1 \\
1984 & 146.6 & 155.5 & 94.3 \\
1985 & 159.4 & 160.7 & 99.2 \\
1986 & 124.4 & 126.8 & 98.2 \\
1987 & 108.2 & 109.5 & 98.8 \\
1988 & 108.6 & 106.4 & 102.1 \\
1989 & 114.5 & 113.0 & 101.4 \\
1990 & 100.0 & 100.0 & 100.0 \\
1991 & 103.5 & 102.0 & 101.5 \\
1992 & 102.9 & 97.8 & 105.2 \\
1993 & 131.3 & 105.6 & 124.4 \\
1994 & 134.6 & 104.3 & 129.0 \\
\hline
\end{tabular}

\begin{tabular}{|l|c|c|}
\cline { 2 - 3 } \multicolumn{1}{c|}{} & \multicolumn{2}{c|}{ Trade Weights } \\
\cline { 2 - 3 } \multicolumn{1}{c|}{} & 1970 & 1994 \\
\hline Australia & 0.7 & 0.6 \\
Austria & 2.6 & 3.4 \\
Belgium & 5.8 & 5.7 \\
Canada & 1.3 & 0.9 \\
Denmark & 1.2 & 1.2 \\
Finland & 0.7 & 0.9 \\
France & 20.3 & 18.3 \\
Western Germany & 32.7 & 29.4 \\
Greece & 0.6 & 1.1 \\
Italy & - & - \\
Japan & 2.6 & 5.2 \\
Netherlands & 5.9 & 5.5 \\
Norway & 0.8 & 0.7 \\
Portugal & 0.4 & 0.8 \\
Spain & 1.8 & 5.9 \\
Sweden & 2.5 & 2.2 \\
United Kingdom & 6.5 & 9.2 \\
United States & 13.6 & 9.0 \\
\hline
\end{tabular}


Trade-Weighted Output per Employed Person and Unit Labour Costs in Total Manufacturing, 1979-94 (Indexes: 1990=100)

Competitors: Australia, Austria, Belgium, Canada, Denmark, Finland, France, Germany, Greece, Italy, Netherlands, Norway, Portugal, Spain, Sweden, United Kingdom, United States

\begin{tabular}{|c|c|c|c|}
\hline \multirow{2}{*}{ Year } & \multicolumn{3}{|c|}{ Output per Employed Person } \\
& \multicolumn{3}{|c|}{ U.S. Dollar Basis } \\
\cline { 2 - 4 } & Own & Competitors' & Ratio \\
\hline 1979 & 67.2 & 75.2 & 89.4 \\
1980 & 68.2 & 74.2 & 92.0 \\
1981 & 69.9 & 76.8 & 91.0 \\
1982 & 72.6 & 77.7 & 93.4 \\
1983 & 73.6 & 82.6 & 89.1 \\
1984 & 76.9 & 87.0 & 88.4 \\
1985 & 82.4 & 89.9 & 91.6 \\
1986 & 81.5 & 90.5 & 90.1 \\
1987 & 86.3 & 94.4 & 91.4 \\
1988 & 91.2 & 98.4 & 92.6 \\
1989 & 95.1 & 99.2 & 95.9 \\
1990 & 100.0 & 100.0 & 100.0 \\
1991 & 102.0 & 100.5 & 101.5 \\
1992 & 99.5 & 103.2 & 96.4 \\
1993 & 97.6 & 105.6 & 92.5 \\
1994 & 98.3 & 111.5 & 88.2 \\
\hline
\end{tabular}

\begin{tabular}{|c|r|c|c|}
\hline \multirow{2}{*}{ Year } & \multicolumn{3}{|c|}{ Unit Labour Costs } \\
& \multicolumn{3}{|c|}{ National Currency Basis } \\
\cline { 2 - 4 } & Own & Competitors' & Ratio \\
\hline 1979 & 88.4 & 67.0 & 132.1 \\
1980 & 92.7 & 75.5 & 122.7 \\
1981 & 96.6 & 80.6 & 119.9 \\
1982 & 98.0 & 86.6 & 113.1 \\
1983 & 99.9 & 86.6 & 115.4 \\
1984 & 100.6 & 87.0 & 115.7 \\
1985 & 98.4 & 89.2 & 110.3 \\
1986 & 103.6 & 92.6 & 111.9 \\
1987 & 100.4 & 92.5 & 108.5 \\
1988 & 97.7 & 92.8 & 105.3 \\
1989 & 98.5 & 95.7 & 102.9 \\
1990 & 100.0 & 100.0 & 100.0 \\
1991 & 101.9 & 104.8 & 97.3 \\
1992 & 106.3 & 107.7 & 98.7 \\
1993 & 111.6 & 108.6 & 102.7 \\
1994 & 113.4 & 106.4 & 106.5 \\
\hline
\end{tabular}

\begin{tabular}{|c|r|c|c|}
\hline \multirow{2}{*}{ Year } & \multicolumn{3}{|c|}{ Unit Labour Costs } \\
& \multicolumn{3}{|c|}{ U.S. Dollar Basis } \\
\cline { 2 - 4 } & Own & Competitors' & Ratio \\
\hline 1979 & 58.4 & 70.7 & 82.7 \\
1980 & 59.2 & 80.0 & 74.0 \\
1981 & 63.4 & 80.4 & 78.9 \\
1982 & 56.9 & 82.7 & 68.9 \\
1983 & 60.9 & 80.0 & 76.2 \\
1984 & 61.3 & 78.0 & 78.7 \\
1985 & 59.7 & 77.4 & 77.2 \\
1986 & 89.0 & 83.9 & 106.0 \\
1987 & 100.5 & 88.0 & 114.2 \\
1988 & 110.4 & 90.1 & 122.4 \\
1989 & 103.3 & 91.2 & 113.4 \\
1990 & 100.0 & 100.0 & 100.0 \\
1991 & 109.6 & 103.8 & 105.6 \\
1992 & 121.5 & 107.9 & 112.6 \\
1993 & 145.2 & 103.6 & 140.2 \\
1994 & 160.6 & 101.9 & 157.6 \\
\hline
\end{tabular}

\begin{tabular}{|c|c|c|c|}
\hline \multirow{2}{*}{ Year } & \multicolumn{3}{|c|}{ Exchange } \\
& \multicolumn{3}{|c|}{ Rates } \\
\cline { 2 - 4 } & Own & $\begin{array}{c}\text { Competitors' } \\
\text { Index }\end{array}$ & Ratio \\
\hline 1979 & 151.4 & 94.8 & 159.7 \\
1980 & 156.6 & 94.5 & 165.8 \\
1981 & 152.3 & 101.6 & 149.9 \\
1982 & 172.0 & 107.2 & 160.5 \\
1983 & 164.0 & 112.1 & 146.3 \\
1984 & 164.0 & 117.8 & 139.3 \\
1985 & 164.7 & 122.4 & 134.6 \\
1986 & 116.4 & 112.4 & 103.6 \\
1987 & 99.9 & 105.4 & 94.8 \\
1988 & 88.5 & 103.1 & 85.9 \\
1989 & 95.3 & 105.4 & 90.4 \\
1990 & 100.0 & 100.0 & 100.0 \\
1991 & 93.0 & 101.0 & 92.1 \\
1992 & 87.5 & 99.9 & 87.5 \\
1993 & 76.8 & 105.6 & 72.7 \\
1994 & 70.6 & 105.3 & 67.0 \\
\hline
\end{tabular}

\begin{tabular}{|l|c|c|}
\cline { 2 - 3 } \multicolumn{1}{c|}{} & \multicolumn{2}{c|}{ Trade Weights } \\
\cline { 2 - 3 } \multicolumn{1}{c|}{} & 1970 & 1994 \\
\hline Australia & 8.0 & 4.9 \\
Austria & 0.4 & 0.8 \\
Belgium & 1.6 & 2.0 \\
Canada & 6.2 & 5.2 \\
Denmark & 0.5 & 1.6 \\
Finland & 0.2 & 0.7 \\
France & 3.1 & 5.5 \\
Western Germany & 11.0 & 13.2 \\
Greece & 0.1 & 0.1 \\
Italy & 2.8 & 4.9 \\
Japan & - & - \\
Netherlands & 1.5 & 1.6 \\
Norway & 0.4 & 0.6 \\
Portugal & 0.2 & 0.1 \\
Spain & 0.4 & 1.1 \\
Sweden & 1.3 & 1.9 \\
United Kingdom & 7.1 & 5.3 \\
United States & 55.1 & 50.5 \\
\hline
\end{tabular}


Trade-Weighted Output per Employed Person and Unit Labour Costs in Total Manufacturing, 1979-94 (Indexes: 1990=100)

Competitors: Australia, Austria, Belgium, Canada, Denmark, Finland, France, Germany, Greece, Italy, Japan, Norway, Portugal, Spain, Sweden, United Kingdom, United States

\begin{tabular}{|c|c|c|r|}
\hline \multirow{2}{*}{ Year } & \multicolumn{3}{|c|}{ Output per Employed Person } \\
\cline { 2 - 4 } & Own & Competitors' & Ratio \\
\hline & Index & Index & 97.6 \\
1979 & 73.6 & 75.4 & 100.2 \\
1980 & 75.0 & 74.9 & 101.0 \\
1981 & 77.4 & 76.6 & 101.0 \\
1982 & 79.3 & 78.5 & 103.5 \\
1983 & 85.7 & 82.9 & 105.6 \\
1984 & 91.1 & 86.3 & 103.8 \\
1985 & 92.4 & 88.9 & 104.5 \\
1986 & 93.9 & 89.9 & 99.7 \\
1987 & 91.2 & 91.4 & 98.5 \\
1988 & 94.2 & 95.7 & 99.8 \\
1989 & 98.2 & 98.4 & 100.0 \\
1990 & 100.0 & 100.0 & 98.9 \\
1991 & 100.2 & 101.3 & 98.5 \\
1992 & 101.1 & 102.7 & 99.5 \\
1993 & 102.7 & 103.2 & 102.0 \\
1994 & 111.6 & 109.5 & \\
\hline
\end{tabular}

\begin{tabular}{|c|r|c|c|}
\hline \multirow{2}{*}{ Year } & \multicolumn{3}{|c|}{ Unit Labour Costs } \\
& \multicolumn{3}{|c|}{ National Currency Basis } \\
\cline { 2 - 4 } & Own & Competitors' & Ratio \\
\hline 1979 & 94.6 & 65.3 & 144.8 \\
1980 & 98.2 & 72.6 & 135.2 \\
1981 & 99.9 & 77.2 & 129.4 \\
1982 & 104.4 & 81.4 & 128.2 \\
1983 & 100.8 & 82.6 & 122.1 \\
1984 & 97.0 & 84.8 & 114.4 \\
1985 & 98.2 & 87.4 & 112.4 \\
1986 & 100.1 & 90.7 & 110.3 \\
1987 & 105.9 & 93.7 & 112.9 \\
1988 & 102.9 & 93.7 & 109.8 \\
1989 & 99.2 & 95.6 & 103.8 \\
1990 & 100.0 & 100.0 & 100.0 \\
1991 & 104.4 & 104.9 & 99.5 \\
1992 & 108.4 & 109.0 & 99.4 \\
1993 & 109.7 & 111.6 & 98.3 \\
1994 & 104.1 & 109.2 & 95.4 \\
\hline
\end{tabular}

\begin{tabular}{|c|c|c|c|}
\hline \multirow[t]{2}{*}{ Year } & \multicolumn{3}{|c|}{$\begin{array}{l}\text { Unit Labour Costs } \\
\text { U.S. Dollar Basis }\end{array}$} \\
\hline & $\begin{array}{l}\text { Own } \\
\text { Index }\end{array}$ & $\begin{array}{l}\text { Competitors' } \\
\text { Index }\end{array}$ & Ratio \\
\hline 1979 & 85.8 & 68.8 & 124.7 \\
\hline 1980 & 89.9 & 77.4 & 116.2 \\
\hline 1981 & 72.9 & 69.3 & 105.2 \\
\hline 1982 & 71.2 & 65.4 & 108.8 \\
\hline 1983 & 64.3 & 61.1 & 105.3 \\
\hline 1984 & 55.0 & 57.3 & 96.1 \\
\hline 1985 & 53.8 & 57.1 & 94.3 \\
\hline 1986 & 74.4 & 72.8 & 102.1 \\
\hline 1987 & 95.2 & 86.2 & 110.4 \\
\hline 1988 & 94.8 & 89.0 & 106.5 \\
\hline 1989 & 85.2 & 85.6 & 99.5 \\
\hline 1990 & 100.0 & 100.0 & 100.0 \\
\hline 1991 & 101.7 & 103.1 & 98.7 \\
\hline 1992 & 112.3 & 111.4 & 100.8 \\
\hline 1993 & 107.5 & 105.8 & 101.7 \\
\hline 1994 & 104.2 & 105.5 & 98.8 \\
\hline
\end{tabular}

\begin{tabular}{|c|c|c|c|}
\hline \multirow{2}{*}{ Year } & \multicolumn{3}{|c|}{$\begin{array}{c}\text { Exchange } \\
\text { Rates }\end{array}$} \\
\cline { 2 - 4 } & Own & $\begin{array}{c}\text { Competitors' } \\
\text { Index }\end{array}$ & Ratio \\
\hline 1979 & 110.2 & 95.9 & 114.8 \\
1980 & 109.2 & 95.4 & 114.4 \\
1981 & 137.0 & 114.0 & 120.2 \\
1982 & 146.6 & 127.4 & 115.1 \\
1983 & 156.7 & 138.5 & 113.1 \\
1984 & 176.2 & 153.2 & 115.0 \\
1985 & 182.4 & 158.8 & 114.9 \\
1986 & 134.5 & 125.7 & 107.1 \\
1987 & 111.2 & 108.9 & 102.1 \\
1988 & 108.5 & 105.6 & 102.8 \\
1989 & 116.5 & 112.2 & 103.8 \\
1990 & 100.0 & 100.0 & 100.0 \\
1991 & 102.7 & 101.9 & 100.8 \\
1992 & 96.6 & 98.0 & 98.5 \\
1993 & 102.0 & 107.2 & 95.1 \\
1994 & 99.9 & 105.9 & 94.4 \\
\hline
\end{tabular}

\begin{tabular}{|l|c|c|}
\cline { 2 - 3 } \multicolumn{1}{c|}{} & \multicolumn{2}{c|}{ Trade Weights } \\
\cline { 2 - 3 } \multicolumn{1}{c|}{} & 1970 & 1994 \\
\hline Australia & 0.5 & 0.5 \\
Austria & 0.9 & 1.7 \\
Belgium & 17.3 & 8.8 \\
Canada & 1.3 & 0.7 \\
Denmark & 0.9 & 1.5 \\
Finland & 0.9 & 1.1 \\
France & 10.1 & 12.4 \\
Western Germany & 33.9 & 30.1 \\
Greece & 0.4 & 0.7 \\
Italy & 6.6 & 7.4 \\
Japan & 3.0 & 6.4 \\
Netherlands & - & - \\
Norway & 0.8 & 0.9 \\
Portugal & 0.3 & 0.9 \\
Spain & 1.2 & 3.0 \\
Sweden & 2.8 & 2.5 \\
United Kingdom & 8.7 & 11.4 \\
United States & 10.4 & 10.0 \\
\hline
\end{tabular}


Trade-Weighted Output per Employed Person and Unit Labour Costs in Total Manufacturing, 1979-94 (Indexes: 1990=100)

Competitors: Australia, Austria, Belgium, Canada, Denmark, Finland, France, Germany, Greece, Italy, Japan, Netherlands, Portugal, Spain, Sweden, United Kingdom, United States

\begin{tabular}{|c|c|c|r|}
\hline \multirow{2}{*}{ Year } & \multicolumn{3}{|c|}{ Output per Employed Person } \\
\cline { 2 - 4 } & Own & Competitors' & Ratio \\
\hline & Index & Index & 102.9 \\
& 77.8 & 75.6 & 101.4 \\
1980 & 77.1 & 76.0 & 99.9 \\
1981 & 77.2 & 77.3 & 100.2 \\
1982 & 79.7 & 79.5 & 99.2 \\
1983 & 84.0 & 84.7 & 101.3 \\
1984 & 89.4 & 88.2 & 101.7 \\
1985 & 91.7 & 90.1 & 99.6 \\
1986 & 90.6 & 90.9 & 100.2 \\
1987 & 92.5 & 92.3 & 95.9 \\
1988 & 92.1 & 96.0 & 98.6 \\
1989 & 97.2 & 98.6 & 100.0 \\
1990 & 100.0 & 100.0 & 99.9 \\
1991 & 101.3 & 101.4 & 106.9 \\
1992 & 111.3 & 104.2 & 106.3 \\
1993 & 113.9 & 107.1 & 104.5 \\
1994 & 119.5 & 114.4 &
\end{tabular}

\begin{tabular}{|c|r|r|r|}
\hline \multirow{2}{*}{ Year } & \multicolumn{3}{|c|}{ Unit Labour Costs } \\
& \multicolumn{3}{|c|}{ National Currency Basis } \\
\cline { 2 - 4 } & $\begin{array}{c}\text { Own } \\
\text { Index }\end{array}$ & $\begin{array}{c}\text { Competitors' } \\
\text { Index }\end{array}$ & Ratio \\
\hline 1979 & 50.8 & 62.4 & 81.5 \\
1980 & 56.2 & 69.0 & 81.4 \\
1981 & 62.4 & 74.9 & 83.2 \\
1982 & 66.7 & 78.9 & 84.5 \\
1983 & 69.9 & 79.1 & 88.3 \\
1984 & 71.6 & 81.4 & 88.0 \\
1985 & 76.0 & 84.5 & 90.0 \\
1986 & 84.4 & 88.4 & 95.4 \\
1987 & 91.7 & 91.3 & 100.5 \\
1988 & 97.8 & 92.2 & 106.2 \\
1989 & 97.1 & 94.9 & 102.3 \\
1990 & 100.0 & 100.0 & 100.0 \\
1991 & 103.9 & 105.3 & 98.7 \\
1992 & 98.7 & 107.7 & 91.6 \\
1993 & 101.1 & 107.6 & 93.9 \\
1994 & 103.1 & 105.2 & 98.0 \\
\hline
\end{tabular}

\begin{tabular}{|c|r|c|r|}
\hline \multirow{2}{*}{ Year } & \multicolumn{3}{|c|}{ Unit Labour Costs } \\
& \multicolumn{3}{|c|}{ U.S. Dollar Basis } \\
\cline { 2 - 4 } & Own & Competitors' & Ratio \\
\hline 1979 & 62.8 & 67.9 & 92.5 \\
1980 & 71.2 & 76.2 & 93.4 \\
1981 & 68.0 & 69.0 & 98.6 \\
1982 & 64.7 & 63.9 & 101.2 \\
1983 & 59.9 & 58.6 & 102.3 \\
1984 & 54.9 & 55.4 & 99.2 \\
1985 & 55.3 & 55.3 & 100.0 \\
1986 & 71.4 & 71.4 & 100.0 \\
1987 & 85.2 & 84.2 & 101.2 \\
1988 & 94.0 & 88.1 & 106.6 \\
1989 & 88.0 & 85.8 & 102.6 \\
1990 & 100.0 & 100.0 & 100.0 \\
1991 & 100.3 & 103.6 & 96.8 \\
1992 & 99.4 & 109.8 & 90.5 \\
1993 & 89.2 & 99.6 & 89.6 \\
1994 & 91.5 & 98.9 & 92.5 \\
\hline
\end{tabular}

\begin{tabular}{|c|r|c|r|}
\hline \multirow{2}{*}{ Year } & \multicolumn{3}{|c|}{ Exchange } \\
& Rates \\
\cline { 2 - 4 } & Own & Competitors' & Ratio \\
\hline 1979 & 80.9 & 92.6 & 87.4 \\
1980 & 78.9 & 91.9 & 85.8 \\
1981 & 91.7 & 110.9 & 82.7 \\
1982 & 103.1 & 125.7 & 82.1 \\
1983 & 116.6 & 137.3 & 84.9 \\
1984 & 130.4 & 150.8 & 86.5 \\
1985 & 137.3 & 156.6 & 87.7 \\
1986 & 118.1 & 124.7 & 94.8 \\
1987 & 107.6 & 108.6 & 99.2 \\
1988 & 104.1 & 104.9 & 99.3 \\
1989 & 110.3 & 111.0 & 99.4 \\
1990 & 100.0 & 100.0 & 100.0 \\
1991 & 103.6 & 101.7 & 101.9 \\
1992 & 99.3 & 98.5 & 100.8 \\
1993 & 113.3 & 111.3 & 101.8 \\
1994 & 112.7 & 110.1 & 102.4 \\
\hline
\end{tabular}

\begin{tabular}{|l|c|c|}
\cline { 2 - 3 } \multicolumn{1}{c|}{} & \multicolumn{2}{c|}{ Trade Weights } \\
\cline { 2 - 3 } \multicolumn{1}{c|}{} & 1970 & 1994 \\
\hline Australia & 0.2 & 0.3 \\
Austria & 1.4 & 1.2 \\
Belgium & 3.3 & 2.9 \\
Canada & 0.7 & 1.8 \\
Denmark & 7.8 & 8.6 \\
Finland & 3.0 & 3.9 \\
France & 4.1 & 5.9 \\
Western Germany & 19.8 & 17.8 \\
Greece & 0.7 & 0.3 \\
Italy & 3.0 & 4.7 \\
Japan & 5.9 & 7.1 \\
Netherlands & 3.3 & 4.3 \\
Norway & - & - \\
Portugal & 0.5 & 1.1 \\
Spain & 0.7 & 1.5 \\
Sweden & 22.6 & 18.4 \\
United Kingdom & 16.7 & 12.8 \\
United States & 6.1 & 7.7 \\
\hline
\end{tabular}


Trade-Weighted Output per Employed Person and Unit Labour Costs in Total Manufacturing, 1979-94 (Indexes: 1990=100)

Competitors: Australia, Austria, Belgium, Canada, Denmark, Finland, France, Germany, Greece, Italy, Japan, Netherlands, Norway, Spain, Sweden, United Kingdom, United States

\begin{tabular}{|c|r|c|r|}
\hline \multirow{2}{*}{ Year } & \multicolumn{3}{|c|}{ Output per Employed Person } \\
\cline { 2 - 4 } & Own & Competitors' & Ratio \\
\hline & Index & Index & 92.4 \\
1979 & 68.7 & 74.4 & 99.2 \\
1980 & 73.1 & 73.7 & 97.0 \\
1981 & 73.8 & 76.1 & 98.9 \\
1982 & 77.2 & 78.1 & 93.8 \\
1983 & 77.2 & 82.3 & 89.8 \\
1984 & 77.3 & 86.1 & 92.2 \\
1985 & 82.0 & 89.0 & 95.6 \\
1986 & 86.4 & 90.3 & 95.8 \\
1987 & 88.2 & 92.0 & 96.4 \\
1988 & 92.8 & 96.2 & 99.2 \\
1989 & 98.1 & 98.9 & 100.0 \\
1990 & 100.0 & 100.0 & 97.7 \\
1991 & 99.0 & 101.4 & 100.3 \\
1992 & 103.5 & 103.2 & 104.4 \\
1993 & 107.6 & 103.0 & 99.2 \\
1994 & 108.9 & 109.8 & \\
\hline
\end{tabular}

\begin{tabular}{|c|r|c|c|}
\hline \multirow{2}{*}{ Year } & \multicolumn{3}{|c|}{ Unit Labour Costs } \\
& \multicolumn{3}{|c|}{ National Currency Basis } \\
\cline { 2 - 4 } & $\begin{array}{c}\text { Own } \\
\text { Index }\end{array}$ & $\begin{array}{c}\text { Competitors' } \\
\text { Index }\end{array}$ & Ratio \\
\hline 1979 & 22.9 & 62.3 & 36.8 \\
1980 & 26.6 & 70.1 & 37.9 \\
1981 & 31.8 & 75.0 & 42.4 \\
1982 & 36.6 & 80.2 & 45.6 \\
1983 & 43.0 & 83.0 & 51.8 \\
1984 & 51.5 & 84.1 & 61.3 \\
1985 & 59.1 & 86.8 & 68.1 \\
1986 & 67.7 & 89.8 & 75.4 \\
1987 & 76.0 & 92.8 & 81.9 \\
1988 & 80.8 & 92.9 & 87.0 \\
1989 & 86.3 & 95.0 & 90.9 \\
1990 & 100.0 & 100.0 & 100.0 \\
1991 & 113.0 & 105.1 & 107.5 \\
1992 & 125.5 & 108.9 & 115.3 \\
1993 & 125.4 & 112.2 & 111.8 \\
1994 & 126.9 & 110.6 & 114.8 \\
\hline
\end{tabular}

\begin{tabular}{|c|r|c|r|}
\hline \multirow{2}{*}{ Year } & \multicolumn{3}{|c|}{ Unit Labour Costs } \\
& \multicolumn{3}{|c|}{ U.S. Dollar Basis } \\
\cline { 2 - 4 } & Own & Competitors' & Ratio \\
\hline 1979 & 66.8 & 68.1 & 98.0 \\
1980 & 75.7 & 77.5 & 97.7 \\
1981 & 73.6 & 69.1 & 106.5 \\
1982 & 65.6 & 65.1 & 100.9 \\
1983 & 55.3 & 62.1 & 89.0 \\
1984 & 50.2 & 56.3 & 89.2 \\
1985 & 49.4 & 55.6 & 88.9 \\
1986 & 64.5 & 71.1 & 90.7 \\
1987 & 76.9 & 84.2 & 91.3 \\
1988 & 80.0 & 87.1 & 91.9 \\
1989 & 78.2 & 84.1 & 92.9 \\
1990 & 100.0 & 100.0 & 100.0 \\
1991 & 111.5 & 102.9 & 108.3 \\
1992 & 132.5 & 110.4 & 120.0 \\
1993 & 111.2 & 102.1 & 108.9 \\
1994 & 109.0 & 100.6 & 108.4 \\
\hline
\end{tabular}

\begin{tabular}{|c|r|c|r|}
\hline \multirow{2}{*}{ Year } & \multicolumn{3}{|c|}{ Exchange } \\
& \multicolumn{3}{|c|}{ Rates } \\
\cline { 2 - 4 } & Own & $\begin{array}{c}\text { Competitors' } \\
\text { Index }\end{array}$ & Ratio \\
\hline 1979 & 34.3 & 92.0 & 37.3 \\
1980 & 35.1 & 91.7 & 38.3 \\
1981 & 43.2 & 110.3 & 39.1 \\
1982 & 55.7 & 125.2 & 44.5 \\
1983 & 77.7 & 136.6 & 56.9 \\
1984 & 102.7 & 153.3 & 67.0 \\
1985 & 119.5 & 160.2 & 74.6 \\
1986 & 104.9 & 127.2 & 82.5 \\
1987 & 98.8 & 110.5 & 89.4 \\
1988 & 101.0 & 107.1 & 94.3 \\
1989 & 110.5 & 113.3 & 97.5 \\
1990 & 100.0 & 100.0 & 100.0 \\
1991 & 101.4 & 102.1 & 99.2 \\
1992 & 94.7 & 98.7 & 95.9 \\
1993 & 112.8 & 111.6 & 101.1 \\
1994 & 116.4 & 112.2 & 103.8 \\
\hline
\end{tabular}

\begin{tabular}{|l|c|c|}
\cline { 2 - 3 } \multicolumn{1}{c|}{} & \multicolumn{2}{c|}{ Trade Weights } \\
\cline { 2 - 3 } \multicolumn{1}{c|}{} & 1970 & 1994 \\
\hline Australia & 0.4 & 0.2 \\
Austria & 2.4 & 1.1 \\
Belgium & 3.6 & 4.6 \\
Canada & 1.2 & 0.4 \\
Denmark & 1.7 & 1.2 \\
Finland & 1.1 & 0.9 \\
France & 11.2 & 15.3 \\
Western Germany & 21.8 & 18.6 \\
Greece & 0.1 & 0.2 \\
Italy & 8.6 & 10.3 \\
Japan & 3.8 & 3.7 \\
Netherlands & 2.8 & 4.3 \\
Norway & 1.2 & 1.3 \\
Portugal & - & - \\
Spain & 5.1 & 20.8 \\
Sweden & 4.4 & 1.8 \\
United Kingdom & 20.0 & 9.8 \\
United States & 10.5 & 5.7 \\
\hline
\end{tabular}


Trade-Weighted Output per Employed Person and Unit Labour Costs in Total Manufacturing, 1979-94 (Indexes: 1990=100)

Competitors: Australia, Austria, Belgium, Canada, Denmark, Finland, France, Germany, Greece, Italy, Japan, Netherlands, Norway, Portugal, Sweden, United Kingdom, United States

\begin{tabular}{|c|c|c|c|}
\hline \multirow{2}{*}{ Year } & \multicolumn{3}{|c|}{ Output per Employed Person } \\
\cline { 2 - 4 } & $\begin{array}{c}\text { Own } \\
\text { Index }\end{array}$ & $\begin{array}{c}\text { Competitors' } \\
\text { Index }\end{array}$ & Ratio \\
\hline 1979 & 71.0 & 74.6 & 95.2 \\
1980 & 72.1 & 74.2 & 97.2 \\
1981 & 77.2 & 76.3 & 101.3 \\
1982 & 81.0 & 77.9 & 104.0 \\
1983 & 84.2 & 81.9 & 102.8 \\
1984 & 88.4 & 85.4 & 103.5 \\
1985 & 94.7 & 88.0 & 107.7 \\
1986 & 96.7 & 89.1 & 108.5 \\
1987 & 98.2 & 90.8 & 108.1 \\
1988 & 100.6 & 95.4 & 105.4 \\
1989 & 101.3 & 98.4 & 103.0 \\
1990 & 100.0 & 100.0 & 100.0 \\
1991 & 102.5 & 100.9 & 101.6 \\
1992 & 105.2 & 102.5 & 102.6 \\
1993 & 100.9 & 103.6 & 97.5 \\
1994 & 108.8 & 109.9 & 99.0 \\
\hline
\end{tabular}

\begin{tabular}{|c|c|c|c|}
\hline \multirow{2}{*}{ Year } & \multicolumn{3}{|c|}{ Unit Labour Costs } \\
& \multicolumn{3}{|c|}{ National Currency Basis } \\
\cline { 2 - 4 } & Own & Competitors' & Ratio \\
\hline 1979 & 46.6 & 64.2 & 72.6 \\
1980 & 55.5 & 71.2 & 77.9 \\
1981 & 59.8 & 76.8 & 77.8 \\
1982 & 65.2 & 82.0 & 79.5 \\
1983 & 70.6 & 83.6 & 84.5 \\
1984 & 73.5 & 85.6 & 85.8 \\
1985 & 74.9 & 88.7 & 84.4 \\
1986 & 79.5 & 91.5 & 86.9 \\
1987 & 82.8 & 94.2 & 87.9 \\
1988 & 85.7 & 93.7 & 91.4 \\
1989 & 91.0 & 95.5 & 95.2 \\
1990 & 100.0 & 100.0 & 100.0 \\
1991 & 105.0 & 105.4 & 99.6 \\
1992 & 110.1 & 109.5 & 100.6 \\
1993 & 118.4 & 111.5 & 106.1 \\
1994 & 120.4 & 109.0 & 110.5 \\
\hline
\end{tabular}

\begin{tabular}{|c|r|c|r|}
\hline \multirow{2}{*}{ Year } & \multicolumn{3}{|c|}{ Unit Labour Costs } \\
& \multicolumn{3}{|c|}{ U.S. Dollar Basis } \\
\cline { 2 - 4 } & Own & Competitors' & Ratio \\
\hline 1979 & 70.7 & 68.5 & 103.3 \\
1980 & 78.9 & 77.4 & 101.9 \\
1981 & 66.0 & 70.3 & 93.9 \\
1982 & 60.5 & 66.7 & 90.7 \\
1983 & 50.2 & 62.6 & 80.2 \\
1984 & 46.6 & 58.4 & 79.8 \\
1985 & 44.9 & 58.9 & 76.2 \\
1986 & 57.9 & 73.9 & 78.3 \\
1987 & 68.4 & 86.9 & 78.7 \\
1988 & 75.0 & 89.0 & 84.2 \\
1989 & 78.3 & 85.2 & 91.9 \\
1990 & 100.0 & 100.0 & 100.0 \\
1991 & 103.0 & 103.3 & 99.7 \\
1992 & 109.6 & 111.8 & 98.0 \\
1993 & 94.8 & 104.4 & 90.8 \\
1994 & 91.6 & 103.3 & 88.7 \\
\hline
\end{tabular}

\begin{tabular}{|c|c|c|c|}
\hline \multirow{2}{*}{ Year } & \multicolumn{3}{|c|}{ Exchange } \\
& Rates \\
\cline { 2 - 4 } & Own & $\begin{array}{c}\text { Competitors' } \\
\text { Index }\end{array}$ & Ratio \\
\hline 1979 & 65.9 & 94.0 & 70.1 \\
1980 & 70.3 & 93.1 & 75.5 \\
1981 & 90.6 & 111.4 & 81.3 \\
1982 & 107.8 & 125.7 & 85.7 \\
1983 & 140.7 & 136.6 & 103.0 \\
1984 & 157.7 & 151.4 & 104.1 \\
1985 & 166.8 & 156.2 & 106.8 \\
1986 & 137.4 & 124.7 & 110.1 \\
1987 & 121.1 & 108.4 & 111.7 \\
1988 & 114.3 & 105.6 & 108.2 \\
1989 & 116.1 & 112.5 & 103.3 \\
1990 & 100.0 & 100.0 & 100.0 \\
1991 & 101.9 & 102.1 & 99.8 \\
1992 & 100.4 & 98.1 & 102.4 \\
1993 & 124.8 & 108.5 & 115.1 \\
1994 & 131.4 & 107.5 & 122.2 \\
\hline
\end{tabular}

\begin{tabular}{|l|c|c|}
\cline { 2 - 3 } \multicolumn{1}{c|}{} & \multicolumn{2}{c|}{ Trade Weights } \\
\cline { 2 - 3 } \multicolumn{1}{c|}{} & 1970 & 1994 \\
\hline Australia & 0.3 & 0.2 \\
Austria & 0.9 & 1.5 \\
Belgium & 4.0 & 5.5 \\
Canada & 1.8 & 0.4 \\
Denmark & 0.8 & 0.9 \\
Finland & 0.9 & 1.0 \\
France & 14.9 & 23.1 \\
Western Germany & 21.2 & 20.4 \\
Greece & 0.2 & 0.4 \\
Italy & 9.2 & 13.2 \\
Japan & 4.4 & 4.2 \\
Netherlands & 4.6 & 4.5 \\
Norway & 0.8 & 0.4 \\
Portugal & 0.6 & 4.5 \\
Spain & - & - \\
Sweden & 3.6 & 1.8 \\
United Kingdom & 11.9 & 10.4 \\
United States & 19.8 & 7.7 \\
\hline
\end{tabular}


Trade-Weighted Output per Employed Person and Unit Labour Costs in Total Manufacturing, 1979-94 (Indexes: 1990=100)

Competitors: Australia, Austria, Belgium, Canada, Denmark, Finland, France, Germany, Greece, Italy, Japan, Netherlands, Norway, Portugal, Spain, United Kingdom, United States

\begin{tabular}{|c|c|c|c|}
\hline \multirow{2}{*}{ Year } & \multicolumn{3}{|c|}{ Output per Employed Person } \\
\cline { 2 - 4 } & Own & Competitors' & Ratio \\
\cline { 2 - 4 } & Index & 75.3 & 103.6 \\
\hline 1979 & 78.0 & 75.0 & 104.7 \\
1980 & 78.6 & 76.8 & 102.7 \\
1981 & 78.8 & 78.5 & 104.8 \\
1982 & 82.3 & 83.0 & 106.9 \\
1983 & 88.8 & 86.4 & 109.3 \\
1984 & 94.5 & 88.9 & 106.0 \\
1985 & 94.2 & 89.8 & 105.8 \\
1986 & 95.0 & 91.5 & 104.1 \\
1987 & 95.3 & 95.6 & 100.8 \\
1988 & 96.3 & 98.5 & 99.7 \\
1989 & 98.1 & 100.0 & 100.0 \\
1990 & 100.0 & 100.9 & 102.5 \\
1991 & 103.4 & 103.5 & 106.1 \\
1992 & 109.8 & 105.0 & 114.4 \\
1993 & 120.2 & 111.1 & 120.7 \\
1994 & 134.1 &
\end{tabular}

\begin{tabular}{|c|r|r|r|}
\hline \multirow{2}{*}{ Year } & \multicolumn{3}{|c|}{ Unit Labour Costs } \\
& \multicolumn{3}{|c|}{ National Currency Basis } \\
\cline { 2 - 4 } & $\begin{array}{c}\text { Own } \\
\text { Index }\end{array}$ & $\begin{array}{c}\text { Competitors' } \\
\text { Index }\end{array}$ & Ratio \\
\hline 1979 & 51.6 & 64.0 & 80.6 \\
1980 & 56.2 & 70.9 & 79.3 \\
1981 & 62.1 & 75.9 & 81.8 \\
1982 & 64.0 & 80.9 & 79.0 \\
1983 & 64.7 & 82.0 & 78.9 \\
1984 & 67.3 & 84.0 & 80.1 \\
1985 & 73.2 & 86.8 & 84.4 \\
1986 & 77.7 & 90.4 & 85.9 \\
1987 & 81.0 & 93.7 & 86.5 \\
1988 & 86.3 & 94.0 & 91.7 \\
1989 & 92.8 & 95.6 & 97.1 \\
1990 & 100.0 & 100.0 & 100.0 \\
1991 & 107.9 & 104.6 & 103.1 \\
1992 & 106.9 & 107.3 & 99.6 \\
1993 & 99.3 & 109.1 & 91.0 \\
1994 & 95.5 & 107.0 & 89.3 \\
\hline
\end{tabular}

\begin{tabular}{|c|r|c|c|}
\hline \multirow{2}{*}{ Year } & \multicolumn{3}{|c|}{ Unit Labour Costs } \\
& \multicolumn{3}{|c|}{ U.S. Dollar Basis } \\
\cline { 2 - 4 } & Own & Competitors' & Ratio \\
\hline 1979 & 71.3 & 66.5 & 107.2 \\
1980 & 78.7 & 74.9 & 105.1 \\
1981 & 72.5 & 68.3 & 106.3 \\
1982 & 60.3 & 65.4 & 92.1 \\
1983 & 49.9 & 61.2 & 81.6 \\
1984 & 48.1 & 57.5 & 83.7 \\
1985 & 50.4 & 57.8 & 87.1 \\
1986 & 64.6 & 73.0 & 88.4 \\
1987 & 75.6 & 86.2 & 87.8 \\
1988 & 83.3 & 89.8 & 92.8 \\
1989 & 85.2 & 86.1 & 99.0 \\
1990 & 100.0 & 100.0 & 100.0 \\
1991 & 105.6 & 102.6 & 102.9 \\
1992 & 108.6 & 108.7 & 99.9 \\
1993 & 75.5 & 102.7 & 73.5 \\
1994 & 73.3 & 102.9 & 71.2 \\
\hline
\end{tabular}

\begin{tabular}{|c|r|c|r|}
\hline \multirow{2}{*}{ Year } & \multicolumn{3}{|c|}{ Exchange } \\
& \multicolumn{3}{|c|}{ Rates } \\
\cline { 2 - 4 } & Own & Competitors' & Ratio \\
\hline 1979 & 72.4 & 96.7 & 74.9 \\
1980 & 71.5 & 95.8 & 74.6 \\
1981 & 85.5 & 113.4 & 75.4 \\
1982 & 106.1 & 126.5 & 83.9 \\
1983 & 129.5 & 137.2 & 94.4 \\
1984 & 139.8 & 151.1 & 92.5 \\
1985 & 145.4 & 155.7 & 93.3 \\
1986 & 120.4 & 125.1 & 96.2 \\
1987 & 107.1 & 109.0 & 98.3 \\
1988 & 103.5 & 105.2 & 98.4 \\
1989 & 108.9 & 111.5 & 97.7 \\
1990 & 100.0 & 100.0 & 100.0 \\
1991 & 102.2 & 102.0 & 100.2 \\
1992 & 98.4 & 99.1 & 99.3 \\
1993 & 131.5 & 108.7 & 121.0 \\
1994 & 130.4 & 106.7 & 122.2 \\
\hline
\end{tabular}

\begin{tabular}{|l|c|c|}
\cline { 2 - 3 } \multicolumn{1}{c|}{} & \multicolumn{2}{c|}{ Trade Weights } \\
\cline { 2 - 3 } \multicolumn{1}{c|}{} & 1970 & 1994 \\
\hline Australia & 0.5 & 0.9 \\
Austria & 2.4 & 1.8 \\
Belgium & 4.1 & 4.1 \\
Canada & 1.1 & 0.9 \\
Denmark & 9.5 & 7.2 \\
Finland & 6.5 & 6.3 \\
France & 5.8 & 7.8 \\
Western Germany & 23.1 & 22.6 \\
Greece & 0.2 & 0.4 \\
Italy & 4.0 & 5.7 \\
Japan & 2.6 & 6.5 \\
Netherlands & 4.0 & 4.7 \\
Norway & 7.6 & 6.0 \\
Portugal & 0.9 & 0.9 \\
Spain & 0.9 & 2.0 \\
Sweden & - & - \\
United Kingdom & 16.8 & 12.1 \\
United States & 10.1 & 10.1 \\
\hline
\end{tabular}


Trade-Weighted Output per Employed Person and Unit Labour Costs in Total Manufacturing, 1979-94 (Indexes: 1990=100)

Competitors: Australia, Austria, Belgium, Canada, Denmark, Finland, France, Germany, Greece, Italy, Japan, Netherlands, Norway, Portugal, Spain, Sweden, United States

\begin{tabular}{|c|c|c|r|}
\hline \multirow{2}{*}{ Year } & \multicolumn{3}{|c|}{ Output per Employed Person } \\
\cline { 2 - 4 } & Own & Competitors' & Ratio \\
\hline & Index & Index & 88.4 \\
& 66.6 & 75.3 & 84.3 \\
1980 & 63.5 & 75.4 & 86.1 \\
1981 & 66.4 & 77.2 & 89.6 \\
1982 & 70.6 & 78.8 & 92.5 \\
1983 & 76.8 & 83.1 & 93.3 \\
1984 & 80.9 & 86.8 & 93.3 \\
1985 & 83.5 & 89.5 & 96.0 \\
1986 & 86.6 & 90.2 & 99.5 \\
1987 & 91.2 & 91.7 & 100.4 \\
1988 & 96.2 & 95.8 & 101.6 \\
1989 & 99.9 & 98.3 & 100.0 \\
1990 & 100.0 & 100.0 & 99.8 \\
1991 & 100.9 & 101.1 & 101.4 \\
1992 & 104.1 & 102.7 & 101.7 \\
1993 & 105.5 & 103.8 & 97.0 \\
1994 & 107.2 & 110.5 & \\
\hline
\end{tabular}

\begin{tabular}{|c|r|r|r|}
\hline \multirow{2}{*}{ Year } & \multicolumn{3}{|c|}{ Unit Labour Costs } \\
& \multicolumn{3}{|c|}{ National Currency Basis } \\
\cline { 2 - 4 } & $\begin{array}{c}\text { Own } \\
\text { Index }\end{array}$ & $\begin{array}{c}\text { Competitors' } \\
\text { Index }\end{array}$ & Ratio \\
\hline 1979 & 54.6 & 66.6 & 82.0 \\
1980 & 66.8 & 73.7 & 90.7 \\
1981 & 74.1 & 78.6 & 94.3 \\
1982 & 77.0 & 83.6 & 92.1 \\
1983 & 76.8 & 84.7 & 90.6 \\
1984 & 79.0 & 86.1 & 91.8 \\
1985 & 83.6 & 88.5 & 94.5 \\
1986 & 87.4 & 91.8 & 95.2 \\
1987 & 92.0 & 94.2 & 97.6 \\
1988 & 92.9 & 94.0 & 98.8 \\
1989 & 93.2 & 96.0 & 97.1 \\
1990 & 100.0 & 100.0 & 100.0 \\
1991 & 106.5 & 104.8 & 101.7 \\
1992 & 107.7 & 108.6 & 99.2 \\
1993 & 107.7 & 110.7 & 97.2 \\
1994 & 105.8 & 108.5 & 97.6 \\
\hline
\end{tabular}

\begin{tabular}{|c|r|c|r|}
\hline \multirow{2}{*}{ Year } & \multicolumn{3}{|c|}{ Unit Labour Costs } \\
& \multicolumn{3}{|c|}{ U.S. Dollar Basis } \\
\cline { 2 - 4 } & Own & Competitors' & \multicolumn{1}{c|}{ Ratio } \\
\hline 1979 & 65.2 & 69.7 & 93.5 \\
1980 & 87.5 & 76.7 & 114.0 \\
1981 & 83.8 & 69.5 & 120.5 \\
1982 & 75.8 & 66.8 & 113.5 \\
1983 & 65.5 & 63.2 & 103.7 \\
1984 & 59.2 & 59.8 & 99.0 \\
1985 & 60.4 & 59.2 & 102.1 \\
1986 & 72.1 & 74.7 & 96.6 \\
1987 & 84.6 & 87.2 & 97.1 \\
1988 & 93.0 & 89.6 & 103.8 \\
1989 & 85.9 & 86.9 & 98.8 \\
1990 & 100.0 & 100.0 & 100.0 \\
1991 & 105.8 & 103.2 & 102.5 \\
1992 & 106.5 & 111.3 & 95.6 \\
1993 & 90.9 & 106.9 & 85.0 \\
1994 & 91.2 & 106.7 & 85.4 \\
\hline
\end{tabular}

\begin{tabular}{|c|r|c|r|}
\hline \multirow{2}{*}{ Year } & \multicolumn{3}{|c|}{ Exchange } \\
& Rates \\
\cline { 2 - 4 } & Own & Competitors' & Ratio \\
\hline 1979 & 83.8 & 96.3 & 87.1 \\
1980 & 76.4 & 97.2 & 78.6 \\
1981 & 88.4 & 114.7 & 77.0 \\
1982 & 101.6 & 128.3 & 79.2 \\
1983 & 117.1 & 138.0 & 84.9 \\
1984 & 133.5 & 150.5 & 88.7 \\
1985 & 138.4 & 156.5 & 88.4 \\
1986 & 121.1 & 124.4 & 97.4 \\
1987 & 108.7 & 108.2 & 100.4 \\
1988 & 99.8 & 105.4 & 94.7 \\
1989 & 108.5 & 111.1 & 97.7 \\
1990 & 100.0 & 100.0 & 100.0 \\
1991 & 100.7 & 101.6 & 99.1 \\
1992 & 101.2 & 97.7 & 103.5 \\
1993 & 118.4 & 105.7 & 112.0 \\
1994 & 116.0 & 104.5 & 111.0 \\
\hline
\end{tabular}

\begin{tabular}{|l|c|c|}
\cline { 2 - 3 } \multicolumn{1}{c|}{} & \multicolumn{2}{c|}{ Trade Weights } \\
\cline { 2 - 3 } \multicolumn{1}{c|}{} & 1970 & 1994 \\
\hline Australia & 4.0 & 1.0 \\
Austria & 1.8 & 1.1 \\
Belgium & 3.9 & 6.4 \\
Canada & 9.2 & 1.3 \\
Denmark & 5.7 & 1.9 \\
Finland & 3.7 & 1.8 \\
France & 6.6 & 14.0 \\
Western Germany & 12.4 & 21.4 \\
Greece & 0.3 & 0.4 \\
Italy & 5.1 & 8.3 \\
Japan & 5.4 & 9.1 \\
Netherlands & 6.1 & 6.9 \\
Norway & 3.7 & 1.4 \\
Portugal & 1.7 & 1.3 \\
Spain & 1.6 & 3.7 \\
Sweden & 7.8 & 3.8 \\
United Kingdom & - & - \\
United States & 21.1 & 16.0 \\
\hline
\end{tabular}


Trade-Weighted Output per Employed Person and Unit Labour Costs in Total Manufacturing, 1979-94 (Indexes: 1990=100)

Competitors: Australia, Austria, Belgium, Canada, Denmark, Finland, France, Germany, Greece, Italy, Japan, Netherlands, Norway, Portugal, Spain, Sweden, United Kingdom

\begin{tabular}{|c|r|c|r|}
\hline \multirow{2}{*}{ Year } & \multicolumn{3}{|c|}{ Output per Employed Person } \\
\cline { 2 - 4 } & Own & Competitors' & Ratio \\
\hline 1979 & 72.1 & 76.3 & 94.5 \\
1980 & 71.2 & 75.0 & 95.0 \\
1981 & 74.6 & 76.9 & 97.0 \\
1982 & 76.0 & 77.1 & 98.6 \\
1983 & 81.6 & 81.2 & 100.5 \\
1984 & 85.9 & 85.9 & 100.0 \\
1985 & 89.2 & 89.4 & 99.7 \\
1986 & 89.6 & 88.8 & 100.9 \\
1987 & 96.0 & 91.4 & 105.0 \\
1988 & 100.4 & 94.9 & 105.7 \\
1989 & 99.6 & 97.4 & 102.3 \\
1990 & 100.0 & 100.0 & 100.0 \\
1991 & 99.6 & 101.2 & 98.4 \\
1992 & 102.8 & 102.5 & 100.4 \\
1993 & 105.7 & 104.2 & 101.4 \\
1994 & 110.7 & 108.8 & 101.7 \\
\hline
\end{tabular}

\begin{tabular}{|c|r|r|r|}
\hline \multirow{2}{*}{ Year } & \multicolumn{3}{|c|}{ Unit Labour Costs } \\
& \multicolumn{3}{|c|}{ National Currency Basis } \\
\cline { 2 - 4 } & $\begin{array}{c}\text { Own } \\
\text { Index }\end{array}$ & $\begin{array}{c}\text { Competitors' } \\
\text { Index }\end{array}$ & Ratio \\
\hline 1979 & 74.7 & 67.3 & 111.0 \\
1980 & 83.8 & 75.1 & 111.6 \\
1981 & 87.8 & 80.8 & 108.7 \\
1982 & 93.2 & 86.5 & 107.8 \\
1983 & 91.4 & 87.7 & 104.2 \\
1984 & 91.1 & 88.1 & 103.3 \\
1985 & 92.6 & 89.2 & 103.8 \\
1986 & 96.0 & 93.9 & 102.2 \\
1987 & 92.5 & 94.7 & 97.6 \\
1988 & 92.5 & 94.8 & 97.6 \\
1989 & 96.2 & 96.6 & 99.6 \\
1990 & 100.0 & 100.0 & 100.0 \\
1991 & 104.7 & 104.3 & 100.4 \\
1992 & 107.5 & 106.9 & 100.6 \\
1993 & 108.1 & 108.3 & 99.8 \\
1994 & 106.7 & 107.2 & 99.6 \\
\hline
\end{tabular}

\begin{tabular}{|c|r|c|c|}
\hline \multirow{2}{*}{ Year } & \multicolumn{3}{|c|}{ Unit Labour Costs } \\
& \multicolumn{3}{|c|}{ U.S. Dollar Basis } \\
\cline { 2 - 4 } & Own & Competitors' & Ratio \\
\hline 1979 & 74.7 & 62.0 & 120.6 \\
1980 & 83.8 & 68.8 & 121.8 \\
1981 & 87.8 & 67.8 & 129.4 \\
1982 & 93.2 & 66.9 & 139.4 \\
1983 & 91.4 & 66.1 & 138.2 \\
1984 & 91.1 & 62.2 & 146.5 \\
1985 & 92.6 & 60.5 & 152.9 \\
1986 & 96.0 & 77.6 & 123.7 \\
1987 & 92.5 & 88.6 & 104.4 \\
1988 & 92.5 & 95.8 & 96.6 \\
1989 & 96.2 & 93.7 & 102.6 \\
1990 & 100.0 & 100.0 & 100.0 \\
1991 & 104.7 & 106.5 & 98.3 \\
1992 & 107.5 & 111.4 & 96.5 \\
1993 & 108.1 & 112.8 & 95.8 \\
1994 & 106.7 & 115.1 & 92.7 \\
\hline
\end{tabular}

\begin{tabular}{|c|c|c|r|}
\hline \multirow{2}{*}{ Year } & \multicolumn{3}{|c|}{$\begin{array}{c}\text { Exchange } \\
\text { Rates }\end{array}$} \\
\cline { 2 - 4 } & Own & $\begin{array}{c}\text { Competitors' } \\
\text { Index }\end{array}$ & Ratio \\
\hline 1979 & 100.0 & 110.0 & 90.9 \\
1980 & 100.0 & 112.5 & 88.8 \\
1981 & 100.0 & 121.0 & 82.6 \\
1982 & 100.0 & 133.3 & 75.0 \\
1983 & 100.0 & 135.9 & 73.6 \\
1984 & 100.0 & 144.9 & 69.0 \\
1985 & 100.0 & 150.1 & 66.6 \\
1986 & 100.0 & 121.5 & 82.3 \\
1987 & 100.0 & 107.5 & 93.0 \\
1988 & 100.0 & 100.0 & 100.0 \\
1989 & 100.0 & 103.8 & 96.3 \\
1990 & 100.0 & 100.0 & 100.0 \\
1991 & 100.0 & 98.1 & 102.0 \\
1992 & 100.0 & 96.5 & 103.6 \\
1993 & 100.0 & 99.8 & 100.2 \\
1994 & 100.0 & 99.4 & 100.6 \\
\hline
\end{tabular}

\begin{tabular}{|l|r|r|}
\cline { 2 - 3 } \multicolumn{1}{c|}{} & \multicolumn{2}{c|}{ Trade Weights } \\
\cline { 2 - 3 } \multicolumn{1}{c|}{} & 1970 & 1994 \\
\hline Australia & 1.7 & 1.0 \\
Austria & 0.5 & 0.5 \\
Belgium & 2.6 & 2.0 \\
Canada & 35.3 & 30.5 \\
Denmark & 1.0 & 0.6 \\
Finland & 0.4 & 0.6 \\
France & 4.0 & 5.1 \\
Western Germany & 12.4 & 10.2 \\
Greece & 0.1 & 0.1 \\
Italy & 5.4 & 4.5 \\
Japan & 23.0 & 33.5 \\
Netherlands & 1.7 & 1.7 \\
Norway & 0.4 & 0.4 \\
Portugal & 0.3 & 0.3 \\
Spain & 1.3 & 1.3 \\
Sweden & 1.7 & 1.4 \\
United Kingdom & 8.2 & 6.3 \\
United States & - & - \\
\hline
\end{tabular}


ANNEX TABLES 19-54

TRADE-WEIGHTED RELATIVE UNIT LABOUR COST BY INDUSTRY

METHODS:

TOTAL PRICE COMPETITIVENESS AND COMPETITIVENESS AMONG EXPORTERS

Tables 19. \& 20.

Tables 21. \& 22.

Tables 23. \& 24.

Tables 25. \& 26.

Tables 27. \& 28.

Tables 29. \& 30 .

Tables 31. \& 32 .

Tables 33. \& 34.

Tables 35. \& 36.

Tables 37. \& 38.

Tables 39. \& 40

Tables 41. \& 42

Tables 43. \& 44.

Tables 45. \& 46.

Tables 47. \& 48.

Tables 49. \& 50.

Tables 51. \& 52.

Tables 53. \& 54.
Australia

Austria

Belgium / Luxembourg Canada Denmark Finland France Western Germany Greece Italy Japan Netherlands Norway Portugal Spain Sweden United Kingdom United States 
Annex Table 19: Trade-Weighted Relative Unit Labour Cost per Industry - Total price competitiveness

Australia

\begin{tabular}{|c|c|c|c|c|c|c|c|c|c|c|c|c|c|c|c|c|}
\hline & 1979 & 1980 & 1981 & 1982 & 1983 & 1984 & 1985 & 1986 & 1987 & 1988 & 1989 & 1990 & 1991 & 1992 & 1993 & 1994 \\
\hline Total manufacturing & 110.2 & 106.9 & 122.8 & 124.9 & 127.8 & 130.3 & 106.5 & 86.7 & 85.2 & 91.6 & 98.5 & 100.0 & 96.6 & 85.8 & 76.2 & 76.3 \\
\hline Food, drink and tobacco & 133.9 & 127.5 & 155.3 & 137.4 & 147.0 & 158.2 & 128.2 & 103.6 & 95.4 & 108.6 & 107.1 & 100.0 & 95.3 & 84.9 & 78.9 & 81.7 \\
\hline Textiles, Footwear and leather & 112.3 & 113.2 & 131.2 & 135.1 & 130.3 & 137.1 & 112.0 & 89.7 & 89.5 & 95.2 & 104.6 & 100.0 & 89.4 & 85.5 & 89.0 & 90.6 \\
\hline Wood, cork and furniture & 76.5 & 79.4 & 89.8 & 91.1 & 88.3 & 98.8 & 76.4 & 77.4 & 71.0 & 80.0 & 93.6 & 100.0 & 94.5 & 91.5 & 87.0 & 83.3 \\
\hline Paper and printing & 117.8 & 112.3 & 126.7 & 120.3 & 128.0 & 129.8 & 106.1 & 90.0 & 89.3 & 94.7 & 103.5 & 100.0 & 101.9 & 102.5 & 96.1 & 88.1 \\
\hline Chemicals & 116.2 & 100.0 & 119.0 & 111.8 & 124.9 & 133.0 & 115.8 & 92.1 & 93.5 & 100.5 & 103.0 & 100.0 & 100.1 & 96.7 & 91.9 & 87.8 \\
\hline Industrial chemicals & 112.2 & 93.8 & 113.1 & 102.8 & 116.5 & 123.6 & 114.1 & 91.2 & 93.0 & 102.7 & 102.6 & 100.0 & 101.7 & 96.8 & 91.7 & 87.6 \\
\hline Pharmaceuticals & 112.5 & 104.1 & 123.4 & 117.7 & 129.3 & 151.0 & 126.6 & 104.6 & 96.0 & 111.2 & 122.0 & 100.0 & 107.4 & 114.2 & 112.5 & 108.3 \\
\hline Petroleum products & 105.1 & 86.6 & 97.4 & 89.6 & 99.9 & 109.7 & 87.5 & 71.1 & 89.3 & 90.7 & 96.1 & 100.0 & 94.0 & 102.2 & 94.8 & 88.3 \\
\hline Rubber and plastic products & 126.6 & 109.0 & 130.0 & 130.8 & 149.5 & 157.8 & 128.7 & 94.1 & 96.9 & 94.6 & 97.8 & 100.0 & 94.8 & 87.8 & 80.1 & 75.5 \\
\hline Stone, clay and glass & 107.7 & 103.3 & 118.1 & 124.5 & 131.1 & 131.5 & 101.5 & 86.1 & 80.7 & 82.0 & 92.5 & 100.0 & 98.9 & 83.1 & 78.4 & 82.1 \\
\hline Basic metals & 151.6 & 151.4 & 182.6 & 204.4 & 155.4 & 166.7 & 136.5 & 98.1 & 100.2 & 104.6 & 100.0 & 100.0 & 95.9 & 91.4 & 80.6 & 84.2 \\
\hline Ferrous metals & 192.0 & 203.4 & 226.5 & 247.5 & 174.1 & 181.9 & 149.1 & 101.8 & 105.1 & 110.9 & 101.1 & 100.0 & 95.9 & 91.5 & 82.8 & 86.7 \\
\hline Non-ferrous metals & 102.6 & 95.1 & 117.8 & 135.2 & 127.2 & 146.9 & 119.5 & 100.6 & 96.0 & 95.1 & 98.9 & 100.0 & 96.3 & 92.2 & 78.0 & 80.8 \\
\hline Fabricated metal products and machinery & 90.3 & 92.5 & 101.3 & 110.6 & 118.3 & 116.6 & 97.2 & 78.6 & 77.3 & 83.3 & 94.7 & 100.0 & 98.7 & 79.2 & 67.8 & 70.7 \\
\hline Fabricated metal products & 87.2 & 85.5 & 104.9 & 120.7 & 120.4 & 125.8 & 96.6 & 81.3 & 74.6 & 79.5 & 93.7 & 100.0 & 97.6 & 87.0 & 81.0 & 84.4 \\
\hline Non-electrical machinery & 77.1 & 75.9 & 83.9 & 90.8 & 97.0 & 92.2 & 83.7 & 75.0 & 78.2 & 85.9 & 104.6 & 100.0 & 91.5 & 67.4 & 60.2 & 60.1 \\
\hline Electrical machinery & 64.2 & 65.4 & 72.5 & 90.1 & 105.1 & 110.5 & 100.5 & 73.8 & 70.5 & 79.0 & 98.6 & 100.0 & 103.2 & 77.2 & 71.1 & 77.4 \\
\hline Shipbuilding & 67.9 & 133.8 & 130.3 & 129.7 & 116.2 & 155.5 & 106.8 & 88.2 & 87.4 & 100.6 & 90.7 & 100.0 & 97.7 & 91.8 & 78.6 & 78.4 \\
\hline Other transport & 281.2 & 263.1 & 295.5 & 381.8 & 585.7 & 662.3 & 527.6 & 508.9 & 511.8 & 511.2 & 351.1 & 100.0 & 46.8 & 47.2 & 21.2 & 12.2 \\
\hline Motor vehicles & 128.8 & 142.4 & 141.9 & 129.3 & 158.7 & 138.8 & 112.8 & 87.3 & 91.1 & 92.3 & 93.5 & 100.0 & 99.9 & 76.9 & 67.2 & 67.2 \\
\hline Aerospace & 86.7 & 83.7 & 79.3 & 74.4 & 92.7 & 93.0 & 69.1 & 71.0 & 86.7 & 83.2 & 90.8 & 100.0 & 115.3 & 113.4 & 108.5 & 108.4 \\
\hline Instruments & 81.6 & 74.7 & 84.6 & 78.7 & 78.0 & 85.0 & 85.0 & 63.7 & 63.0 & 84.4 & 90.9 & 100.0 & 92.8 & 90.0 & 70.1 & 69.0 \\
\hline Other manufacturing industries & 94.4 & 84.6 & 99.5 & 100.9 & 95.3 & 107.1 & 102.7 & 70.8 & 74.2 & 89.4 & 103.6 & 100.0 & 94.9 & 86.2 & 68.2 & 68.1 \\
\hline
\end{tabular}

Annex Table 20: Trade-Weighted Relative Unit Labour Cost per Industry - Competitiveness among exporters

Australia

\begin{tabular}{|c|c|c|c|c|c|c|c|c|c|c|c|c|c|c|c|c|}
\hline & 1979 & 1980 & 1981 & 1982 & 1983 & 1984 & 1985 & 1986 & 1987 & 1988 & 1989 & 1990 & 1991 & 1992 & 1993 & 1994 \\
\hline Total manufacturing & 108.5 & 105.2 & 126.7 & 128.5 & 133.4 & 138.0 & 113.2 & 92.2 & 87.5 & 95.2 & 102.4 & 100.0 & 97.5 & 86.3 & 79.5 & 80.5 \\
\hline Food, drink and tobacco & 120.1 & 117.2 & 148.8 & 133.6 & 144.2 & 156.4 & 127.2 & 103.1 & 92.3 & 107.1 & 108.6 & 100.0 & 97.0 & 85.4 & 81.3 & 85.7 \\
\hline Textiles, Footwear and leather & 103.3 & 104.4 & 128.2 & 127.5 & 126.4 & 133.6 & 110.8 & 92.8 & 90.6 & 98.4 & 108.6 & 100.0 & 90.9 & 87.4 & 95.0 & 97.9 \\
\hline Wood, cork and furniture & 74.9 & 77.0 & 90.4 & 92.0 & 89.8 & 100.3 & 77.2 & 77.6 & 72.5 & 81.2 & 95.9 & 100.0 & 95.0 & 91.8 & 92.9 & 90.5 \\
\hline Paper and printing & 112.8 & 109.4 & 128.6 & 124.1 & 133.1 & 135.0 & 110.6 & 92.9 & 90.4 & 95.9 & 104.2 & 100.0 & 102.0 & 101.5 & 94.5 & 87.3 \\
\hline Chemicals & 114.1 & 100.9 & 125.1 & 120.5 & 136.4 & 150.8 & 127.8 & 98.6 & 94.3 & 103.0 & 106.3 & 100.0 & 100.4 & 95.9 & 92.8 & 89.4 \\
\hline Industrial chemicals & 111.9 & 95.7 & 120.3 & 114.0 & 129.4 & 141.5 & 125.8 & 96.8 & 93.1 & 103.8 & 105.0 & 100.0 & 102.4 & 95.7 & 92.2 & 88.6 \\
\hline Pharmaceuticals & 102.9 & 98.4 & 122.8 & 117.8 & 134.7 & 163.1 & 136.0 & 105.9 & 96.0 & 111.5 & 122.8 & 100.0 & 107.0 & 113.9 & 110.9 & 106.6 \\
\hline Petroleum products & 101.8 & 89.8 & 104.6 & 95.3 & 108.8 & 129.3 & 98.7 & 78.7 & 83.7 & 88.6 & 96.0 & 100.0 & 94.8 & 104.1 & 104.2 & 98.0 \\
\hline Rubber and plastic products & 116.1 & 104.0 & 129.1 & 130.8 & 152.4 & 163.6 & 133.9 & 99.6 & 100.1 & 101.2 & 103.4 & 100.0 & 96.1 & 88.2 & 84.6 & 83.4 \\
\hline Stone, clay and glass & 103.6 & 99.5 & 118.5 & 128.1 & 134.2 & 133.4 & 101.2 & 87.9 & 81.4 & 82.8 & 94.1 & 100.0 & 99.4 & 82.5 & 77.6 & 81.4 \\
\hline Basic metals & 136.0 & 133.9 & 173.3 & 184.8 & 156.1 & 164.7 & 138.4 & 108.2 & 105.4 & 113.4 & 105.5 & 100.0 & 97.9 & 93.5 & 85.8 & 90.8 \\
\hline Ferrous metals & 171.6 & 181.7 & 221.3 & 228.6 & 176.8 & 183.9 & 153.4 & 110.4 & 108.2 & 116.8 & 106.4 & 100.0 & 96.6 & 91.5 & 84.5 & 89.8 \\
\hline Non-ferrous metals & 96.3 & 88.5 & 111.7 & 132.5 & 128.1 & 145.4 & 123.6 & 108.0 & 103.1 & 109.1 & 104.2 & 100.0 & 99.5 & 96.4 & 85.8 & 89.6 \\
\hline Fabricated metal products and machinery & 93.6 & 94.9 & 107.8 & 117.3 & 126.4 & 125.7 & 105.3 & 84.5 & 80.0 & 86.2 & 98.3 & 100.0 & 99.4 & 79.4 & 70.1 & 73.2 \\
\hline Fabricated metal products & 87.0 & 86.2 & 108.5 & 123.8 & 127.3 & 133.1 & 101.8 & 84.5 & 75.8 & 81.4 & 96.2 & 100.0 & 98.1 & 87.2 & 82.6 & 84.8 \\
\hline Non-electrical machinery & 86.0 & 82.8 & 94.6 & 103.1 & 108.5 & 104.6 & 91.9 & 79.5 & 80.7 & 88.7 & 108.3 & 100.0 & 92.4 & 67.4 & 61.2 & 61.4 \\
\hline Electrical machinery & 68.2 & 68.7 & 79.9 & 98.5 & 113.8 & 119.2 & 105.9 & 78.4 & 73.1 & 82.4 & 101.5 & 100.0 & 102.7 & 75.7 & 69.3 & 74.3 \\
\hline Shipbuilding & 82.7 & 121.0 & 134.5 & 126.5 & 140.1 & 167.3 & 115.3 & 100.3 & 88.0 & 91.2 & 102.1 & 100.0 & 102.2 & 102.1 & 88.8 & 88.7 \\
\hline Other transport & 255.0 & 305.2 & 376.1 & 389.5 & 606.6 & 725.4 & 564.3 & 440.4 & 429.1 & 409.5 & 312.7 & 100.0 & 47.0 & 46.7 & 23.9 & 14.1 \\
\hline Motor vehicles & 124.2 & 131.9 & 138.9 & 128.8 & 159.3 & 143.4 & 116.9 & 94.5 & 92.3 & 97.8 & 99.3 & 100.0 & 103.5 & 79.1 & 72.6 & 75.0 \\
\hline Aerospace & 82.0 & 78.9 & 85.0 & 81.6 & 98.9 & 109.9 & 84.6 & 81.2 & 86.2 & 79.2 & 94.6 & 100.0 & 115.9 & 115.0 & 110.5 & 114.8 \\
\hline Instruments & 82.2 & 74.8 & 90.3 & 87.9 & 90.9 & 101.5 & 101.7 & 71.5 & 66.5 & 86.6 & 95.4 & 100.0 & 93.3 & 90.5 & 72.3 & 72.5 \\
\hline Other manufacturing industries & 91.5 & 88.4 & 109.9 & 118.1 & 115.2 & 129.4 & 121.9 & 79.6 & 77.3 & 93.8 & 105.2 & 100.0 & 100.4 & 91.5 & 75.5 & 81.0 \\
\hline
\end{tabular}

Source: OECD, DSTI (STAN Industrial Database), 1996. 
DSTI/DOC(98)1

Annex Table 21: Trade-Weighted Relative Unit Labour Cost per Industry - Total price competitiveness

Austria

\begin{tabular}{|c|c|c|c|c|c|c|c|c|c|c|c|c|c|c|c|c|}
\hline & 1979 & 1980 & 1981 & 1982 & 1983 & 1984 & 1985 & 1986 & 1987 & 1988 & 1989 & 1990 & 1991 & 1992 & 1993 & 1994 \\
\hline Total manufacturing & 100.8 & 99.7 & 97.6 & 99.9 & 102.1 & 98.4 & 98.8 & 104.3 & 105.8 & 99.7 & 97.5 & 100.0 & 99.3 & 100.8 & 103.8 & $\overline{105.4}$ \\
\hline Food, drink and tobacco & 115.0 & 114.7 & 115.1 & 108.7 & 110.8 & 107.0 & 103.1 & 107.9 & 101.6 & 103.7 & 97.5 & 100.0 & 98.9 & 93.4 & 96.2 & 96.1 \\
\hline Textiles, Footwear and leather & 89.9 & 88.4 & 88.6 & 92.6 & 93.1 & 91.3 & 89.2 & 92.3 & 100.3 & 98.5 & 97.3 & 100.0 & 98.6 & 98.1 & 103.9 & 107.2 \\
\hline Wood, cork and furniture & 117.3 & 115.4 & 111.1 & 113.7 & 106.4 & 115.9 & 110.2 & 114.1 & 112.5 & 106.2 & 102.2 & 100.0 & 93.1 & 99.8 & 112.8 & 116.8 \\
\hline Paper and printing & 118.7 & 116.0 & 115.8 & 116.1 & 120.9 & 114.9 & 110.2 & 116.0 & 118.7 & 103.0 & 96.5 & 100.0 & 99.4 & 104.0 & 106.6 & 103.0 \\
\hline Chemicals & 87.4 & 92.6 & 92.6 & 110.0 & 125.8 & 106.0 & 115.8 & 117.1 & 104.3 & 102.2 & 95.7 & 100.0 & 99.6 & 108.4 & 111.5 & 117.0 \\
\hline Industrial chemicals & 102.6 & 104.4 & 104.9 & 118.4 & 133.0 & 109.6 & 124.5 & 123.1 & 108.1 & 102.9 & 93.6 & 100.0 & 96.9 & 103.0 & 103.0 & 103.6 \\
\hline Pharmaceuticals & 54.1 & 58.4 & 61.3 & 79.4 & 90.1 & 73.7 & 87.9 & 98.0 & 98.5 & 98.3 & 97.0 & 100.0 & 109.7 & 116.1 & 114.2 & 118.0 \\
\hline Petroleum products & 48.8 & 54.6 & 44.8 & 53.7 & 101.8 & 95.5 & 103.4 & 110.7 & 93.3 & 99.8 & 97.0 & 100.0 & 98.3 & 118.7 & 113.7 & 144.5 \\
\hline Rubber and plastic products & 85.9 & 87.6 & 92.5 & 117.1 & 116.7 & 97.1 & 102.9 & 107.5 & 97.4 & 95.8 & 95.9 & 100.0 & 101.1 & 111.9 & 126.2 & 130.4 \\
\hline Stone, clay and glass & 104.1 & 95.7 & 93.5 & 90.4 & 91.2 & 91.9 & 91.3 & 99.6 & 100.0 & 97.9 & 97.7 & 100.0 & 93.6 & 99.4 & 104.5 & 103.3 \\
\hline Basic metals & 151.7 & 137.1 & 135.7 & 134.1 & 135.3 & 128.3 & 127.1 & 126.9 & 134.2 & 103.3 & 98.6 & 100.0 & 95.2 & 105.1 & 113.8 & 113.8 \\
\hline Ferrous metals & 151.6 & 140.5 & 139.5 & 133.9 & 138.8 & 132.2 & 130.7 & 126.5 & 135.7 & 103.7 & 100.3 & 100.0 & 95.4 & 107.1 & 116.6 & 120.5 \\
\hline Non-ferrous metals & 154.1 & 127.5 & 120.5 & 130.4 & 115.6 & 113.5 & 115.3 & 126.2 & 127.3 & 103.0 & 93.3 & 100.0 & 96.2 & 98.2 & 109.2 & 100.2 \\
\hline Fabricated metal products and machinery & 86.8 & 87.7 & 83.4 & 84.0 & 84.4 & 84.0 & 85.5 & 93.4 & 101.9 & 95.6 & 97.1 & 100.0 & 102.1 & 101.1 & 101.9 & 103.2 \\
\hline Fabricated metal products & 115.7 & 96.2 & 95.4 & 96.9 & 111.3 & 83.5 & 84.4 & 92.2 & 98.1 & 99.6 & 98.5 & 100.0 & 99.5 & 101.1 & 105.9 & 101.5 \\
\hline Non-electric & .. & .. & .. & & & & & & & .. & .. & .. & .. & .. & .. & .. \\
\hline Electrical machinery & 76.9 & 80.2 & 75.2 & 75.4 & 76.5 & 76.7 & 84.3 & 86.3 & 95.2 & 90.6 & 96.1 & 100.0 & 100.6 & 109.0 & 103.7 & 107.8 \\
\hline Shipbuilding & .. & 138.5 & 108.3 & 102.4 & 84.6 & 94.8 & 85.2 & 98.5 & 119.6 & 99.4 & 89.3 & 100.0 & 94.3 & .. & 53.4 & 47.1 \\
\hline Other transport & 70.9 & 66.4 & 69.7 & 74.8 & 79.0 & 80.3 & 85.7 & 99.1 & 105.1 & 100.2 & 96.6 & 100.0 & 98.2 & 95.8 & 71.1 & 76.5 \\
\hline Motor vehicles & 94.4 & 90.0 & 87.7 & 93.7 & 116.2 & 114.8 & 120.8 & 125.0 & 122.9 & 105.3 & 105.0 & 100.0 & 110.8 & 115.9 & 110.5 & 121.3 \\
\hline Aerospace & .. & .. & .. & .. & .. & .. & .. & .. & .. & .. & .. & .. & .. & .. & .. & .. \\
\hline Instruments & .. & .. & .. & .. & .. & & & .. & .. & .. & .. & .. & .. & .. & .. & .. \\
\hline
\end{tabular}

Annex Table 22: Trade-Weighted Relative Unit Labour Cost per Industry - Competitiveness among exporters

Austria

\begin{tabular}{|c|c|c|c|c|c|c|c|c|c|c|c|c|c|c|c|c|}
\hline & 1979 & 1980 & 1981 & 1982 & 1983 & 1984 & 1985 & 1986 & 1987 & 1988 & 1989 & 1990 & 1991 & 1992 & 1993 & 1994 \\
\hline Total manufacturing & 94.8 & 93.5 & 87.5 & 90.0 & 91.5 & 86.6 & 87.2 & 97.0 & 103.8 & 97.2 & 94.3 & 100.0 & 97.7 & 101.9 & 103.9 & 104.2 \\
\hline Food, drink and tobacco & 110.1 & 110.5 & 105.2 & 102.2 & 99.9 & 92.9 & 91.6 & 103.7 & 101.0 & 102.4 & 96.6 & 100.0 & 99.5 & 97.9 & 99.9 & 102.0 \\
\hline Textiles, Footwear and leather & 87.1 & 86.0 & 84.5 & 89.2 & 90.4 & 87.1 & 85.6 & 91.6 & 100.9 & 98.4 & 96.4 & 100.0 & 98.0 & 98.0 & 104.6 & 110.2 \\
\hline Wood, cork and furniture & 103.4 & 103.7 & 95.0 & 98.9 & 93.7 & 100.7 & 95.9 & 110.1 & 113.8 & 106.1 & 99.9 & 100.0 & 90.9 & 98.6 & 112.6 & 115.5 \\
\hline Paper and printing & 125.9 & 121.9 & 114.9 & 112.5 & 115.8 & 104.5 & 100.3 & 113.6 & 119.6 & 103.7 & 95.2 & 100.0 & 98.3 & 106.9 & 113.3 & 108.6 \\
\hline Chemicals & 78.5 & 80.5 & 78.5 & 92.5 & 108.7 & 89.8 & 100.0 & 105.1 & 102.3 & 99.2 & 92.8 & 100.0 & 99.8 & 111.3 & 112.6 & 116.5 \\
\hline Industrial chemicals & 92.7 & 91.5 & 89.9 & 101.7 & 115.7 & 93.4 & 107.6 & 109.6 & 106.2 & 100.8 & 91.5 & 100.0 & 97.9 & 107.0 & 105.7 & 104.2 \\
\hline Pharmaceuticals & 50.3 & 51.7 & 53.7 & 68.1 & 81.7 & 67.8 & 81.9 & 93.2 & 99.2 & 99.2 & 96.7 & 100.0 & 106.9 & 117.6 & 117.4 & 118.8 \\
\hline Petroleum products & 37.4 & 42.7 & 32.7 & 39.2 & 79.4 & 75.9 & 80.4 & 92.4 & 93.5 & 98.0 & 93.6 & 100.0 & 96.6 & 115.2 & 113.2 & 140.7 \\
\hline Rubber and plastic products & 83.2 & 82.5 & 86.0 & 108.2 & 107.0 & 86.1 & 92.8 & 99.3 & 96.7 & 94.1 & 93.3 & 100.0 & 101.7 & 116.4 & 131.7 & 137.2 \\
\hline Stone, clay and glass & 96.7 & 89.9 & 85.9 & 83.9 & 86.6 & 85.6 & 85.9 & 96.7 & 99.7 & 97.2 & 95.8 & 100.0 & 91.3 & 98.7 & 102.3 & 101.1 \\
\hline Basic metals & 149.6 & 133.2 & 126.9 & 126.1 & 123.4 & 120.6 & 118.3 & 120.4 & 132.3 & 103.1 & 97.5 & 100.0 & 93.6 & 103.0 & 108.5 & 105.8 \\
\hline Ferrous metals & 149.9 & 140.4 & 135.6 & 131.4 & 128.2 & 127.4 & 124.1 & 119.5 & 133.3 & 103.0 & 99.4 & 100.0 & 92.4 & 102.5 & 106.4 & 106.2 \\
\hline Non-ferrous metals & 144.9 & 117.7 & 101.5 & 107.6 & 101.9 & 103.0 & 104.6 & 121.1 & 126.6 & 104.7 & 92.3 & 100.0 & 99.2 & 101.5 & 114.5 & 104.7 \\
\hline Fabricated metal products and machinery & 79.4 & 81.3 & 72.3 & 73.0 & 73.4 & 71.8 & 73.5 & 85.0 & 98.2 & 92.1 & 92.8 & 100.0 & 99.7 & 101.9 & 102.7 & 102.5 \\
\hline Fabricated metal products & 108.7 & 90.5 & 86.6 & 90.1 & 102.3 & 76.1 & 78.3 & 89.3 & 98.2 & 98.4 & 96.0 & 100.0 & 97.3 & 102.4 & 109.4 & 108.5 \\
\hline Non-electrical machinery & 74.5 & 76.8 & 67.5 & 66.4 & 70.8 & 71.6 & 74.8 & 88.3 & 99.9 & 94.4 & 95.2 & 100.0 & 96.7 & 96.9 & 99.3 & 96.1 \\
\hline Electrical machinery & 58.0 & 62.1 & 54.5 & 56.5 & 60.5 & 60.5 & 67.1 & 75.4 & 87.5 & 85.2 & 90.1 & 100.0 & 98.2 & 108.5 & 109.7 & 109.7 \\
\hline Shipbuilding & 91.4 & 118.3 & 95.5 & 96.7 & 78.0 & 97.8 & 88.9 & 93.0 & 100.7 & 99.6 & 88.0 & 100.0 & 93.7 & 50.7 & 47.2 & 35.7 \\
\hline Other transport & 51.5 & 57.3 & 55.3 & 61.5 & 67.3 & 69.9 & 73.0 & 81.1 & 96.2 & 88.6 & 88.5 & 100.0 & 96.8 & 91.4 & 82.7 & 78.9 \\
\hline Motor vehicles & 86.5 & 84.4 & 78.2 & 85.0 & 105.3 & 102.9 & 105.1 & 115.8 & 121.5 & 104.6 & 102.9 & 100.0 & 105.0 & 114.2 & 111.5 & 115.2 \\
\hline Aerospace & 91.0 & 84.7 & 69.0 & 73.9 & 75.5 & 74.0 & 71.1 & 86.8 & 108.8 & 102.4 & 96.7 & 100.0 & 98.3 & 101.4 & 101.9 & 99.2 \\
\hline Instruments & 281.0 & 212.7 & 82.9 & 126.2 & 78.3 & 74.6 & 96.4 & 91.6 & 80.2 & 76.4 & 71.3 & 100.0 & 113.8 & 127.8 & 115.6 & 103.8 \\
\hline ther manufac & 92.4 & 89.0 & 87.0 & 94.4 & 91.4 & 92.9 & 94.1 & 99.0 & 103.5 & 96.8 & 92.9 & 100.0 & 98.0 & 101.5 & 100.4 & 103.7 \\
\hline
\end{tabular}

Source: OECD, DSTI (STAN Industrial Database), 1996. 


\begin{tabular}{|c|c|c|c|c|c|c|c|c|c|c|c|c|c|c|c|c|}
\hline & 1979 & 1980 & 1981 & 1982 & 1983 & 1984 & 1985 & 1986 & 1987 & 1988 & 1989 & 1990 & 1991 & 1992 & 1993 & 1994 \\
\hline Total manufacturing & 139.9 & 130.4 & 115.1 & 97.2 & 92.2 & 90.3 & 90.9 & 96.1 & 98.9 & 96.1 & 94.2 & 100.0 & 101.0 & 102.7 & 101.6 & 101.9 \\
\hline Food, drink and tobacco & 123.6 & 118.8 & 111.7 & 93.2 & 86.9 & 86.2 & 89.3 & 88.1 & 84.3 & 86.7 & 89.3 & 100.0 & 101.1 & 103.5 & 101.0 & 103.6 \\
\hline Textiles, Footwear and leather & 167.0 & 154.6 & 134.6 & 126.5 & 116.8 & 119.7 & 124.8 & 116.9 & 113.3 & 113.1 & 101.7 & 100.0 & 102.2 & 100.4 & 96.2 & 98.4 \\
\hline Wood, cork and furniture & 167.9 & 147.2 & 124.6 & 104.7 & 105.9 & 109.0 & 109.8 & 106.0 & 106.5 & 101.8 & 96.5 & 100.0 & 101.8 & 110.0 & 122.8 & 129.6 \\
\hline Paper and printing & 154.0 & 157.9 & 139.0 & 118.0 & 109.5 & 108.6 & 115.4 & 114.5 & 113.3 & 102.3 & 95.3 & 100.0 & 102.7 & 101.7 & 95.7 & 98.2 \\
\hline Chemicals & 124.5 & 120.4 & 110.3 & 95.9 & 98.0 & 92.4 & 92.2 & 101.4 & 96.4 & 93.0 & 93.2 & 100.0 & 97.6 & 99.5 & 106.0 & .. \\
\hline Industrial chemicals & & & & & & & .. & .. & . & .. & .. & .. &. & .. & & .. \\
\hline Pharmaceuticals & 120.9 & 132.3 & 124.0 & 102.8 & 94.8 & 81.4 & 77.0 & 82.1 & 96.6 & 92.6 & 88.9 & 100.0 & 112.2 & 120.3 & 120.1 & \\
\hline Petroleum products & 122.9 & 97.3 & 90.7 & 90.3 & 108.5 & 90.4 & 95.8 & 85.3 & 79.6 & 71.1 & 76.1 & 100.0 & 100.7 & 128.7 & 135.6 & 178.4 \\
\hline Rubber and plastic products & 162.1 & 153.2 & 143.8 & 120.1 & 121.8 & 123.9 & 116.0 & 124.2 & 110.6 & 103.5 & 100.2 & 100.0 & 96.5 & 89.8 & 108.4 & 112.1 \\
\hline Stone, clay and glass & 173.4 & 154.5 & 136.8 & 113.6 & 106.4 & 112.5 & 119.9 & 110.0 & 104.0 & 94.5 & 93.4 & 100.0 & 106.8 & 99.9 & 96.8 & 98.4 \\
\hline Basic metals & & & & .. & .. & .. & .. & .. & .. & .. & .. & .. & .. & .. & .. & .. \\
\hline Ferrous metals & & & .. & .. & .. & .. & .. & .. & .. & .. & .. & .. & .. & .. & .. & .. \\
\hline Non-ferrous metals & .. & & .. & .. & .. & .. & .. & .. & .. & .. & .. & .. & .. & .. & .. & .. \\
\hline Fabricated metal products and machinery & 131.9 & 123.7 & 106.3 & 85.9 & 80.0 & 78.3 & 77.4 & 86.9 & 99.2 & 97.9 & 94.8 & 100.0 & 102.8 & 105.5 & 100.7 & 100.8 \\
\hline Fabricated metal products & 156.8 & 148.9 & 126.7 & 101.1 & 99.8 & 95.4 & 95.2 & 106.2 & 112.6 & 108.1 & 96.3 & 100.0 & 103.2 & 103.1 & 86.4 & 85.5 \\
\hline Non-electrical machinery & .. & .. & .. & .. & .. & .. & .. & .. & .. & .. & .. & .. & .. & .. & .. & .. \\
\hline Electrical machinery & .. & .. & .. & .. & .. & .. & .. & .. & .. & .. & .. & .. & .. & .. & .. & .. \\
\hline Shipbuilding & .. & & .. & .. & .. & .. & .. & .. & .. & .. & .. & .. & .. & .. & .. & .. \\
\hline Other transport & .. & .. & .. & .. & .. & .. & .. & .. & .. & .. & .. & .. & .. & .. & .. & .. \\
\hline Motor vehicles & .. & .. & .. & .. & & & .. & & .. & .. & .. & .. & .. & .. & .. & \\
\hline Aerospace & .. & .. & .. & .. & .. & .. & .. & .. & .. & .. & .. & .. & .. & .. & .. & .. \\
\hline Instruments & .. & .. & .. & .. & .. & .. & .. & .. & .. & .. & .. & .. & .. & .. & .. & \\
\hline
\end{tabular}

Annex Table 24: Trade-Weighted Relative Unit Labour Cost per Industry - Competitiveness among exporters

Belgium / Luxembourg

\begin{tabular}{|c|c|c|c|c|c|c|c|c|c|c|c|c|c|c|c|c|}
\hline & 1979 & 1980 & 1981 & 1982 & 1983 & 1984 & 1985 & 1986 & 1987 & 1988 & 1989 & 1990 & 1991 & 1992 & 1993 & 1994 \\
\hline Total manufacturing & 143.1 & 134.2 & 113.8 & 95.3 & 88.9 & 86.1 & 87.5 & 93.7 & 98.8 & 94.6 & 91.6 & 100.0 & 100.1 & 102.9 & 100.2 & 99.6 \\
\hline Food, drink and tobacco & 130.7 & 124.6 & 111.2 & 91.3 & 84.1 & 82.0 & 86.7 & 88.9 & 88.3 & 89.1 & 88.8 & 100.0 & 100.8 & 104.9 & 103.4 & 105.8 \\
\hline Textiles, Footwear and leather & 169.0 & 158.3 & 135.0 & 125.1 & 115.4 & 118.8 & 126.9 & 118.7 & 114.8 & 114.7 & 102.0 & 100.0 & 102.1 & 99.9 & 96.4 & 99.2 \\
\hline Wood, cork and furniture & 178.4 & 154.6 & 125.4 & 105.5 & 106.5 & 106.7 & 108.6 & 109.4 & 110.8 & 103.4 & 95.0 & 100.0 & 101.4 & 113.5 & 133.0 & 138.0 \\
\hline Paper and printing & 167.0 & 168.2 & 140.4 & 116.0 & 108.3 & 104.3 & 110.4 & 116.4 & 118.4 & 103.9 & 93.3 & 100.0 & 103.2 & 105.6 & 101.3 & 102.5 \\
\hline Chemicals & 122.4 & 118.1 & 106.6 & 91.8 & 92.9 & 87.8 & 88.9 & 98.3 & 96.3 & 91.7 & 91.5 & 100.0 & 97.3 & 99.9 & 104.8 & 102.8 \\
\hline Industrial chemicals & 101.8 & 98.8 & 88.3 & 76.2 & 77.9 & 76.0 & 80.9 & 92.4 & 90.5 & 88.5 & 91.4 & 100.0 & 94.8 & 97.6 & 98.0 & 92.2 \\
\hline Pharmaceuticals & 126.9 & 135.7 & 122.8 & 101.1 & 93.4 & 79.0 & 74.6 & 81.2 & 98.3 & 93.4 & 88.8 & 100.0 & 112.3 & 121.7 & 122.9 & 127.6 \\
\hline Petroleum products & 120.1 & 95.6 & 87.8 & 86.7 & 102.4 & 86.2 & 87.8 & 82.7 & 85.3 & 72.4 & 75.3 & 100.0 & 97.0 & 120.5 & 128.6 & 168.1 \\
\hline Rubber and plastic products & 169.5 & 159.4 & 144.8 & 119.9 & 119.4 & 118.1 & 113.8 & 122.6 & 112.5 & 103.6 & 98.1 & 100.0 & 95.9 & 90.8 & 110.2 & 112.5 \\
\hline Stone, clay and glass & 188.6 & 165.6 & 140.2 & 114.3 & 107.6 & 109.8 & 118.1 & 109.6 & 105.6 & 94.5 & 92.0 & 100.0 & 105.4 & 100.6 & 98.6 & 99.1 \\
\hline Basic metals & 133.2 & 130.4 & 115.4 & 105.1 & 97.7 & 96.0 & 97.4 & 100.6 & 99.4 & 92.2 & 93.5 & 100.0 & 104.3 & 113.0 & 121.1 & 119.1 \\
\hline Ferrous metals & 144.6 & 141.7 & 113.0 & 114.1 & 105.4 & 103.3 & 103.0 & 103.4 & 99.1 & 96.2 & 96.0 & 100.0 & 105.3 & 122.0 & 132.5 & 133.5 \\
\hline Non-ferrous metals & 105.8 & 116.0 & 128.0 & 91.4 & 83.5 & 84.2 & 89.0 & 96.5 & 101.9 & 85.4 & 89.4 & 100.0 & 102.9 & 96.1 & 99.6 & 93.8 \\
\hline Fabricated metal products and machinery & 128.1 & 122.5 & 100.3 & 80.6 & 73.9 & 71.5 & 71.7 & 81.8 & 96.1 & 94.3 & 91.0 & 100.0 & 101.4 & 105.5 & 98.2 & 97.7 \\
\hline Fabricated metal products & 156.7 & 149.0 & 122.5 & 98.7 & 95.6 & 89.8 & 92.2 & 105.3 & 114.1 & 107.7 & 94.1 & 100.0 & 102.2 & 104.6 & 89.8 & 88.6 \\
\hline Non-electrical machinery & 119.1 & 115.1 & 93.4 & 73.4 & 71.7 & 71.6 & 72.8 & 84.8 & 97.5 & 96.3 & 93.3 & 100.0 & 98.4 & 100.1 & 94.6 & 90.6 \\
\hline Electrical machinery & 97.6 & 98.4 & 82.0 & 68.3 & 64.4 & 64.9 & 68.0 & 78.1 & 90.9 & 91.4 & 91.2 & 100.0 & 107.3 & 113.7 & 106.8 & 108.7 \\
\hline Shipbuilding & 101.4 & 127.6 & 100.1 & 82.7 & 68.7 & 70.8 & 65.6 & 74.2 & 80.0 & 84.9 & 86.5 & 100.0 & 99.1 & 104.9 & 92.7 & 84.6 \\
\hline Other transport & 81.9 & 85.0 & 76.3 & 68.1 & 65.7 & 67.8 & 70.9 & 77.5 & 94.7 & 91.5 & 87.5 & 100.0 & 98.3 & 94.6 & 79.3 & 80.5 \\
\hline Motor vehicles & 147.0 & 133.7 & 115.8 & 93.2 & 85.7 & 79.7 & 79.7 & 87.3 & 101.9 & 97.1 & 92.4 & 100.0 & 99.1 & 102.9 & 92.7 & 91.0 \\
\hline Aerospace & 148.1 & 129.5 & 94.4 & 84.0 & 76.5 & 74.5 & 66.7 & 82.9 & 106.1 & 103.8 & 94.4 & 100.0 & 100.5 & 104.2 & 96.8 & 94.9 \\
\hline Instruments & 112.2 & 84.9 & 68.3 & 48.9 & 39.5 & 41.7 & 42.0 & 53.1 & 75.4 & 79.6 & 97.6 & 100.0 & 101.2 & 115.4 & 171.2 & 147.7 \\
\hline 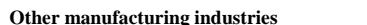 & 172.2 & 106.4 & 85.2 & 73.0 & 61.0 & 60.5 & 60.2 & 78.8 & 90.7 & 89.8 & 105.1 & 100.0 & 84.6 & 87.0 & 102.0 & \\
\hline
\end{tabular}

Source: OECD, DSTI (STAN Industrial Database), 1996. 
DSTI/DOC(98)1

Annex Table 25: Trade-Weighted Relative Unit Labour Cost per Industry - Total price competitiveness

Canada

\begin{tabular}{|c|c|c|c|c|c|c|c|c|c|c|c|c|c|c|c|c|}
\hline & 1979 & 1980 & 1981 & 1982 & 1983 & 1984 & 1985 & 1986 & 1987 & 1988 & 1989 & 1990 & 1991 & 1992 & 1993 & 1994 \\
\hline Total manufacturing & 79.9 & 82.4 & 86.0 & 93.5 & 94.3 & 87.4 & 84.1 & 80.9 & 85.9 & 94.7 & 100.0 & 100.0 & 102.9 & 93.1 & 83.7 & 77.6 \\
\hline Food, drink and tobacco & 85.3 & 88.1 & 96.6 & 103.4 & 107.1 & 102.2 & 102.5 & 95.3 & 92.2 & 102.3 & 107.4 & 100.0 & 98.1 & 90.4 & 81.9 & 76.2 \\
\hline Textiles, Footwear and leather & 81.2 & 84.6 & 85.7 & 97.1 & 98.7 & 97.2 & 98.3 & 87.1 & 85.1 & 94.0 & 101.2 & 100.0 & 103.5 & 94.8 & 90.4 & 84.7 \\
\hline Wood, cork and furniture & 93.5 & 95.5 & 93.0 & 105.8 & 101.1 & 94.0 & 84.9 & 83.2 & 87.7 & 93.3 & 99.1 & 100.0 & 101.5 & 85.9 & 73.5 & 71.9 \\
\hline Paper and printing & 85.3 & 82.1 & 87.3 & 98.2 & 96.0 & 89.4 & 87.5 & 83.4 & 84.8 & 93.7 & 101.4 & 100.0 & 104.9 & 95.5 & 88.0 & 78.6 \\
\hline Chemicals & 78.9 & 77.7 & 86.2 & 94.6 & 95.0 & 85.8 & 86.4 & 85.1 & 92.1 & 98.3 & 102.6 & 100.0 & 105.1 & 93.3 & 83.8 & 77.6 \\
\hline Industrial chemicals & 75.4 & 72.9 & 82.4 & 91.6 & 91.7 & 84.3 & 85.9 & 85.2 & 94.4 & 98.4 & 102.4 & 100.0 & 104.2 & 95.0 & 84.8 & 78.6 \\
\hline Pharmaceuticals & 70.0 & 62.0 & 69.7 & 78.1 & 83.2 & 81.7 & 86.6 & 85.6 & 92.3 & 102.3 & 101.7 & 100.0 & 116.7 & 109.3 & 103.1 & 99.2 \\
\hline Petroleum products & 80.0 & 77.6 & 86.6 & 94.8 & 97.4 & 86.3 & 94.9 & 86.6 & 106.7 & 109.3 & 123.2 & 100.0 & 92.9 & 83.0 & 82.4 & 69.7 \\
\hline Rubber and plastic products & 81.4 & 88.2 & 92.8 & 97.6 & 97.7 & 87.0 & 81.7 & 81.4 & 83.3 & 90.4 & 96.0 & 100.0 & 106.2 & 90.2 & 77.5 & 72.9 \\
\hline Stone, clay and glass & 68.0 & 72.8 & 76.1 & 82.6 & 88.3 & 82.9 & 81.4 & 81.3 & 77.9 & 85.3 & 95.1 & 100.0 & 106.3 & 99.1 & 86.4 & 75.5 \\
\hline Basic metals & 86.0 & 101.4 & 99.7 & 124.7 & 105.0 & 105.1 & 95.0 & 96.5 & 87.1 & 93.7 & 100.4 & 100.0 & 104.8 & 94.7 & 83.0 & 73.9 \\
\hline Ferrous metals & 70.8 & 89.5 & 82.8 & 120.7 & 93.3 & 99.9 & 93.9 & 93.3 & 88.7 & 94.7 & 98.8 & 100.0 & 112.5 & 98.0 & 86.5 & 78.9 \\
\hline Non-ferrous metals & 124.8 & 131.7 & 136.6 & 142.8 & 127.1 & 117.1 & 99.9 & 106.8 & 88.2 & 93.9 & 102.8 & 100.0 & 99.6 & 92.9 & 79.8 & 69.3 \\
\hline Fabricated metal products and machinery & 80.0 & 84.0 & 84.9 & 91.1 & 95.1 & 86.2 & 81.8 & 77.6 & 86.5 & 95.2 & 98.5 & 100.0 & 104.2 & 94.6 & 84.7 & 78.0 \\
\hline Fabricated metal products & 76.4 & 79.0 & 81.2 & 89.3 & 95.0 & 89.0 & 83.6 & 77.9 & 81.2 & 95.3 & 99.5 & 100.0 & 107.8 & 97.0 & 89.2 & 84.0 \\
\hline Non-electrical machinery & 74.4 & 80.2 & 80.9 & 88.9 & 96.2 & 78.1 & 74.9 & 72.4 & 78.1 & 91.3 & 100.1 & 100.0 & 98.1 & 85.0 & 75.3 & 63.2 \\
\hline Electrical machinery & 74.8 & 82.6 & 80.9 & 84.5 & 93.9 & 85.0 & 79.5 & 74.4 & 77.6 & 90.0 & 97.9 & 100.0 & 109.2 & 95.8 & 92.9 & 88.7 \\
\hline Shipbuilding & 134.9 & 167.5 & 204.1 & 239.3 & 140.9 & 103.1 & 98.0 & 85.7 & 77.8 & 85.0 & 95.7 & 100.0 & 93.1 & 87.7 & 63.4 & 42.2 \\
\hline Other transport & 59.0 & 67.7 & 72.8 & 105.6 & 100.1 & 81.5 & 91.9 & 100.2 & 140.4 & 130.1 & 116.2 & 100.0 & 97.0 & 75.3 & 58.1 & 48.6 \\
\hline Motor vehicles & 105.5 & 93.5 & 105.4 & 104.6 & 123.8 & 126.3 & 118.7 & 107.7 & 119.6 & 111.7 & 105.6 & 100.0 & 95.3 & 94.6 & 88.1 & 85.3 \\
\hline Aerospace & 73.5 & 95.7 & 82.2 & 100.5 & 83.0 & 66.8 & 67.6 & 77.9 & 93.1 & 98.6 & 94.0 & 100.0 & 106.5 & 94.1 & 71.9 & 61.5 \\
\hline Instruments & 73.0 & 71.5 & 78.8 & 76.5 & 89.1 & 79.9 & 76.8 & 69.7 & 71.0 & 88.5 & 92.6 & 100.0 & 97.5 & 79.0 & 65.6 & 57.4 \\
\hline Other manufacturing industries & 60.7 & 60.8 & 65.8 & 66.4 & 68.3 & 74.7 & 76.5 & 77.7 & 72.0 & 88.4 & 103.4 & 100.0 & 91.6 & 76.0 & 69.9 & 66.2 \\
\hline
\end{tabular}

Annex Table 26: Trade-Weighted Relative Unit Labour Cost per Industry - Competitiveness among exporters

Canada

\begin{tabular}{|c|c|c|c|c|c|c|c|c|c|c|c|c|c|c|c|c|}
\hline & 1979 & 1980 & 1981 & 1982 & 1983 & 1984 & 1985 & 1986 & 1987 & 1988 & 1989 & 1990 & 1991 & 1992 & 1993 & 1994 \\
\hline $\begin{array}{l}\text { Total manufacturing } \\
\end{array}$ & 86.8 & 90.2 & 103.3 & 123.4 & 127.4 & 122.7 & 120.4 & 94.6 & 87.5 & 93.3 & 104.7 & 100.0 & 102.9 & 89.5 & 80.0 & $\overline{71.4}$ \\
\hline Food, drink and tobacco & 77.6 & 83.0 & 98.1 & 111.0 & 122.8 & 121.5 & 120.6 & 103.2 & 92.2 & 100.0 & 111.7 & 100.0 & 102.2 & 93.6 & 91.0 & 82.6 \\
\hline Textiles, Footwear and leather & 88.8 & 92.2 & 106.8 & 127.5 & 130.6 & 129.4 & 129.5 & 97.8 & 90.0 & 98.5 & 107.9 & 100.0 & 102.1 & 88.8 & 90.8 & 84.7 \\
\hline Wood, cork and furniture & 107.9 & 102.3 & 113.6 & 137.3 & 140.2 & 135.6 & 123.0 & 100.0 & 92.1 & 98.4 & 108.6 & 100.0 & 102.0 & 87.5 & 89.2 & 88.2 \\
\hline Paper and printing & 77.6 & 76.3 & 90.8 & 111.6 & 117.7 & 119.0 & 117.3 & 94.7 & 88.2 & 96.5 & 107.4 & 100.0 & 104.8 & 94.3 & 96.1 & 87.8 \\
\hline Chemicals & 87.6 & 89.0 & 109.2 & 132.8 & 133.5 & 129.1 & 126.8 & 103.3 & 91.7 & 97.9 & 108.5 & 100.0 & 104.7 & 91.3 & 85.1 & 77.6 \\
\hline Industrial chemicals & 84.6 & 83.9 & 105.6 & 131.6 & 129.2 & 127.0 & 127.5 & 104.4 & 93.6 & 96.8 & 107.1 & 100.0 & 105.4 & 92.6 & 85.3 & 77.1 \\
\hline Pharmaceuticals & 62.6 & 60.5 & 73.0 & 85.1 & 94.2 & 97.4 & 104.8 & 92.6 & 88.7 & 99.7 & 107.4 & 100.0 & 117.0 & 108.0 & 106.8 & 104.5 \\
\hline Petroleum products & 91.4 & 91.0 & 98.3 & 118.8 & 130.2 & 130.6 & 127.9 & 108.3 & 100.8 & 104.9 & 125.8 & 100.0 & 94.9 & 84.4 & 95.1 & 81.3 \\
\hline Rubber and plastic products & 92.8 & 102.8 & 118.4 & 143.8 & 137.7 & 128.2 & 121.8 & 97.9 & 86.2 & 94.2 & 103.6 & 100.0 & 102.1 & 84.1 & 71.5 & 64.2 \\
\hline Stone, clay and glass & 74.6 & 81.1 & 97.5 & 123.3 & 126.0 & 124.2 & 122.3 & 96.5 & 86.1 & 92.8 & 103.5 & 100.0 & 106.5 & 90.8 & 83.0 & 72.0 \\
\hline Basic metals & 86.4 & 97.2 & 103.9 & 144.4 & 134.2 & 133.6 & 123.3 & 96.6 & 84.2 & 93.6 & 108.3 & 100.0 & 100.9 & 88.7 & 77.2 & 71.9 \\
\hline Ferrous metals & 75.1 & 93.0 & 91.5 & 149.2 & 123.7 & 130.9 & 124.9 & 95.7 & 87.1 & 95.0 & 105.1 & 100.0 & 109.9 & 91.9 & 79.8 & 76.9 \\
\hline Non-ferrous metals & 112.4 & 109.1 & 120.8 & 142.0 & 154.1 & 138.6 & 120.6 & 101.1 & 82.8 & 92.0 & 110.9 & 100.0 & 92.3 & 86.4 & 74.1 & 65.1 \\
\hline Fabricated metal products and machinery & 84.4 & 94.5 & 102.4 & 121.2 & 126.5 & 115.0 & 116.2 & 90.8 & 86.8 & 90.4 & 101.2 & 100.0 & 105.7 & 90.5 & 77.2 & 67.4 \\
\hline Fabricated metal products & 84.8 & 86.7 & 96.4 & 119.0 & 127.0 & 121.6 & 118.5 & 90.0 & 81.9 & 93.3 & 103.3 & 100.0 & 108.3 & 91.3 & 84.6 & 75.1 \\
\hline Non-electrical machinery & 106.6 & 112.1 & 119.8 & 142.6 & 149.1 & 122.6 & 118.7 & 92.3 & 86.1 & 92.5 & 106.3 & 100.0 & 99.6 & 78.8 & 67.6 & 52.9 \\
\hline Electrical machinery & 81.6 & 92.8 & 95.1 & 112.0 & 122.3 & 116.8 & 118.0 & 92.8 & 85.0 & 92.4 & 104.0 & 100.0 & 107.9 & 86.2 & 76.8 & 66.4 \\
\hline Shipbuilding & 102.2 & 159.7 & 199.1 & 235.6 & 153.8 & 113.9 & 104.4 & 86.6 & 56.8 & 74.6 & 90.4 & 100.0 & 98.2 & 102.8 & 77.7 & 55.9 \\
\hline Other transport & 65.5 & 89.7 & 104.8 & 120.2 & 107.8 & 109.4 & 120.0 & 92.6 & 104.1 & 95.8 & 103.5 & 100.0 & 100.4 & 78.5 & 65.1 & 71.3 \\
\hline Motor vehicles & 95.3 & 102.3 & 116.3 & 129.4 & 147.3 & 138.4 & 141.3 & 108.3 & 108.4 & 98.0 & 104.6 & 100.0 & 103.3 & 95.6 & 84.6 & 77.4 \\
\hline Aerospace & 65.8 & 85.1 & 93.1 & 107.5 & 97.2 & 81.2 & 82.3 & 87.8 & 92.7 & 94.0 & 98.5 & 100.0 & 107.7 & 94.8 & 73.8 & 66.1 \\
\hline Instruments & 71.1 & 73.5 & 90.9 & 99.4 & 123.4 & 114.7 & 114.0 & 87.0 & 74.3 & 85.7 & 100.1 & 100.0 & 98.8 & 77.0 & 64.0 & 54.4 \\
\hline ther manufac & 68.3 & 77.7 & 88.5 & 102.3 & 111.7 & 119.7 & 115.4 & 96.7 & 78.9 & 93.0 & 109.1 & 100.0 & 94.8 & 79.9 & 73.3 & 71.3 \\
\hline
\end{tabular}

Source: OECD, DSTI (STAN Industrial Database), 1996. 
Annex Table 27: Trade-Weighted Relative Unit Labour Cost per Industry - Total price competitiveness

Denmark

\begin{tabular}{|c|c|c|c|c|c|c|c|c|c|c|c|c|c|c|c|c|}
\hline & 1979 & 1980 & 1981 & 1982 & 1983 & 1984 & 1985 & 1986 & 1987 & 1988 & 1989 & 1990 & 1991 & 1992 & 1993 & 1994 \\
\hline Total manufacturing & 95.0 & 81.6 & 77.5 & 76.9 & 77.4 & 77.0 & 79.7 & 88.9 & 97.7 & 94.7 & 92.5 & 100.0 & 95.5 & 96.3 & 93.3 & 97.1 \\
\hline Food, drink and tobacco & 138.6 & 119.1 & 113.2 & 109.7 & 105.7 & 106.2 & 99.9 & 102.6 & 107.4 & 101.7 & 98.2 & 100.0 & 87.4 & 84.5 & 83.2 & 87.2 \\
\hline Textiles, Footwear and leather & 86.2 & 75.9 & 69.7 & 73.2 & 70.9 & 71.8 & 75.0 & 85.1 & 95.7 & 94.6 & 90.8 & 100.0 & 91.6 & 101.2 & 104.0 & 105.7 \\
\hline Wood, cork and furniture & 89.3 & 81.4 & 72.9 & 72.7 & 69.5 & 68.1 & 69.1 & 85.1 & 98.7 & 93.4 & 93.8 & 100.0 & 99.8 & 103.3 & 93.8 & 102.4 \\
\hline Paper and printing & 85.9 & 76.2 & 72.2 & 72.9 & 80.7 & 75.9 & 77.9 & 83.3 & 95.9 & 98.6 & 94.2 & 100.0 & 98.7 & 107.0 & 116.3 & 112.4 \\
\hline Chemicals & 105.9 & 73.0 & 71.1 & 71.6 & 70.8 & 81.6 & 80.9 & 90.4 & 97.4 & 94.8 & 94.3 & 100.0 & 101.3 & 101.1 & 105.3 & 100.2 \\
\hline Industrial chemicals & 102.0 & 69.3 & 67.9 & 66.6 & 64.9 & 75.4 & 77.4 & 87.3 & 95.4 & 94.5 & 94.4 & 100.0 & 104.1 & 110.3 & 113.5 & 108.3 \\
\hline Pharmaceuticals & 102.9 & 76.0 & 74.9 & 72.0 & 69.0 & 78.3 & 80.1 & 90.8 & 100.9 & 98.2 & 94.8 & 100.0 & 102.8 & 96.9 & 97.0 & 91.5 \\
\hline Petroleum products & 64.3 & 48.1 & 44.2 & 45.2 & 46.7 & 45.4 & 45.8 & 62.0 & 71.1 & 65.5 & 82.3 & 100.0 & 83.2 & 91.3 & 105.8 & 95.9 \\
\hline Rubber and plastic products & 138.3 & 95.9 & 88.9 & 90.6 & 92.0 & 107.6 & 109.4 & 115.2 & 117.6 & 101.6 & 97.8 & 100.0 & 97.7 & 91.2 & 94.0 & 89.4 \\
\hline Stone, clay and glass & 81.3 & 78.1 & 72.9 & 75.4 & 78.4 & 79.7 & 80.0 & 84.8 & 99.1 & 102.9 & 93.8 & 100.0 & 94.7 & 89.2 & 86.3 & 95.2 \\
\hline Basic metals & .. & .. & .. & .. & .. & .. & .. & .. & .. & .. & .. & .. & .. & .. & .. & .. \\
\hline Ferrous metals & .. & .. & .. & .. & .. & .. & .. & .. & .. & .. & .. & .. & .. & .. & .. & .. \\
\hline Non-ferrous metals & .. & .. & .. & .. & .. & .. & .. & .. & .. & .. & .. & .. & .. & .. & .. & .. \\
\hline Fabricated metal products and machinery & 83.6 & 74.8 & 69.6 & 67.8 & 68.1 & 65.3 & 72.1 & 84.8 & 94.2 & 90.1 & 88.6 & 100.0 & 98.1 & 99.1 & 91.2 & 97.7 \\
\hline Fabricated metal products & 87.3 & 77.7 & 76.2 & 71.8 & 73.8 & 73.9 & 80.3 & 90.7 & 99.0 & 92.7 & 91.3 & 100.0 & 93.3 & 96.7 & 88.2 & 94.1 \\
\hline Non-electrical machinery & 77.6 & 66.6 & 63.5 & 60.7 & 66.4 & 69.5 & 73.1 & 85.9 & 89.8 & 91.5 & 92.5 & 100.0 & 98.5 & 96.2 & 93.6 & 91.8 \\
\hline Electrical machinery & 66.2 & 71.7 & 68.4 & 63.4 & 61.7 & 58.0 & 59.2 & 71.3 & 83.3 & 83.2 & 79.3 & 100.0 & 95.4 & 79.5 & 77.3 & 85.3 \\
\hline Shipbuilding & 83.1 & 87.1 & 75.7 & 84.0 & 63.1 & 56.3 & 71.8 & 85.2 & 84.8 & 85.1 & 86.7 & 100.0 & 92.3 & 106.5 & 69.3 & 81.2 \\
\hline Other transport & 62.3 & 56.5 & 44.1 & 31.5 & 39.9 & 40.8 & 52.4 & 67.5 & 90.5 & 102.6 & 94.6 & 100.0 & 75.9 & 71.1 & 47.0 & 59.1 \\
\hline Motor vehicles & .. & .. & .. & .. & .. & .. & .. & .. & .. & .. & .. & .. & .. & .. & .. & .. \\
\hline Aerospace & .. & .. & .. & .. & .. & .. & .. & .. & .. & & .. & 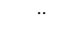 & .. & .. & .. & .. \\
\hline Instruments & .. & .. & .. & .. & .. & .. & .. & .. & .. & .. & .. & .. & .. & .. & .. & .. \\
\hline Other manufacturing industries & 102.9 & 75.5 & 67.5 & 72.1 & 69.1 & 75.5 & 86.1 & 93.5 & 102.6 & 90.1 & 89.7 & 100.0 & 87.2 & 84.9 & 83.5 & 84.9 \\
\hline
\end{tabular}

Annex Table 28: Trade-Weighted Relative Unit Labour Cost per Industry - Competitiveness among exporters

Denmark

\begin{tabular}{|c|c|c|c|c|c|c|c|c|c|c|c|c|c|c|c|c|}
\hline & 1979 & 1980 & 1981 & 1982 & 1983 & 1984 & 1985 & 1986 & 1987 & 1988 & 1989 & 1990 & 1991 & 1992 & 1993 & $\overline{1994}$ \\
\hline Total manufacturing & 94.0 & 82.0 & 76.3 & 73.8 & 72.3 & 71.5 & 74.9 & 84.7 & 95.3 & 92.9 & 90.3 & 100.0 & 94.9 & 95.6 & 88.9 & 91.8 \\
\hline Food, drink and tobacco & 128.5 & 113.6 & 105.7 & 104.0 & 97.6 & 96.2 & 93.3 & 99.0 & 104.9 & 100.2 & 97.8 & 100.0 & 87.8 & 86.0 & 84.1 & 90.1 \\
\hline Textiles, Footwear and leather & 84.3 & 75.7 & 70.4 & 73.5 & 70.0 & 69.8 & 73.5 & 83.4 & 94.1 & 93.9 & 90.4 & 100.0 & 91.5 & 101.2 & 102.0 & 104.9 \\
\hline Wood, cork and furniture & 85.1 & 79.9 & 71.4 & 68.1 & 64.4 & 65.0 & 68.6 & 84.6 & 97.5 & 93.2 & 92.1 & 100.0 & 100.1 & 104.1 & 90.4 & 97.3 \\
\hline Paper and printing & 86.9 & 77.8 & 71.8 & 68.1 & 71.5 & 67.5 & 70.7 & 79.0 & 92.2 & 96.3 & 92.3 & 100.0 & 96.3 & 103.5 & 104.8 & 101.3 \\
\hline Chemicals & 106.1 & 72.9 & 71.3 & 68.4 & 66.2 & 75.7 & 77.1 & 88.0 & 96.2 & 94.5 & 93.9 & 100.0 & 100.9 & 100.6 & 100.1 & 95.3 \\
\hline Industrial chemicals & 99.1 & 66.9 & 66.6 & 63.5 & 61.9 & 70.7 & 74.0 & 85.0 & 95.0 & 93.5 & 94.0 & 100.0 & 103.6 & 110.2 & 109.5 & 105.1 \\
\hline Pharmaceuticals & 100.6 & 72.1 & 71.8 & 68.1 & 66.4 & 75.8 & 78.6 & 91.2 & 100.3 & 99.2 & 95.5 & 100.0 & 103.0 & 96.6 & 94.5 & 89.6 \\
\hline Petroleum products & 72.2 & 55.9 & 49.8 & 48.3 & 44.6 & 44.3 & 47.2 & 62.4 & 71.1 & 71.3 & 85.3 & 100.0 & 90.8 & 96.3 & 99.7 & 93.8 \\
\hline Rubber and plastic products & 132.8 & 90.7 & 86.5 & 86.5 & 86.1 & 99.6 & 102.7 & 109.4 & 115.4 & 101.0 & 97.3 & 100.0 & 97.1 & 90.3 & 89.7 & 85.7 \\
\hline Stone, clay and glass & 82.0 & 79.6 & 73.8 & 72.0 & 73.9 & 73.8 & 76.5 & 81.6 & 96.7 & 101.7 & 91.9 & 100.0 & 95.2 & 91.1 & 84.6 & 94.3 \\
\hline Basic metals & 159.9 & 116.9 & 112.2 & 120.8 & 96.6 & 94.5 & 91.5 & 119.9 & 127.1 & 108.5 & 90.6 & 100.0 & 106.9 & 105.6 & 105.6 & 95.7 \\
\hline Ferrous metals & 153.3 & 114.1 & 110.0 & 114.0 & 90.5 & 91.4 & 91.2 & 111.7 & 119.0 & 105.8 & 89.7 & 100.0 & 107.1 & 105.1 & 105.2 & 96.7 \\
\hline Non-ferrous metals & 183.6 & 134.2 & 123.6 & 146.3 & 117.2 & 108.3 & 96.2 & 144.0 & 150.1 & 116.9 & 94.4 & 100.0 & 107.5 & 106.2 & 105.6 & 93.4 \\
\hline Fabricated metal products and machinery & 81.0 & 74.0 & 67.1 & 64.2 & 63.7 & 61.1 & 67.5 & 80.0 & 91.1 & 87.5 & 85.8 & 100.0 & 97.3 & 98.4 & 87.1 & 92.5 \\
\hline Fabricated metal products & 85.1 & 76.1 & 73.7 & 69.1 & 69.5 & 68.3 & 75.2 & 87.2 & 97.3 & 91.7 & 89.8 & 100.0 & 92.5 & 97.2 & 86.2 & 92.4 \\
\hline Non-electrical machinery & 71.6 & 62.9 & 59.0 & 55.3 & 60.7 & 63.2 & 66.9 & 81.3 & 87.1 & 89.4 & 89.9 & 100.0 & 98.4 & 96.5 & 89.3 & 86.3 \\
\hline Electrical machinery & 59.5 & 67.5 & 63.0 & 60.2 & 58.7 & 57.1 & 62.7 & 68.1 & 78.5 & 79.9 & 77.2 & 100.0 & 94.1 & 77.1 & 75.0 & 82.4 \\
\hline Shipbuilding & 68.2 & 86.2 & 72.7 & 81.2 & 66.5 & 59.6 & 75.4 & 90.6 & 99.2 & 85.2 & 85.0 & 100.0 & 90.4 & 104.5 & 70.9 & 81.8 \\
\hline Other transport & 44.5 & 49.3 & 36.9 & 26.3 & 34.6 & 35.4 & 45.7 & 57.1 & 84.0 & 93.1 & 87.0 & 100.0 & 74.6 & 68.2 & 45.9 & 54.7 \\
\hline Motor vehicles & 142.3 & 129.1 & 97.0 & 66.3 & 68.8 & 62.3 & 80.1 & 98.0 & 139.9 & 111.0 & 100.6 & 100.0 & 95.2 & 105.3 & 75.4 & 90.3 \\
\hline Aerospace & 94.1 & 79.5 & 64.7 & 70.2 & 67.1 & 65.2 & 60.2 & 81.0 & 100.9 & 97.6 & 89.4 & 100.0 & 96.2 & 98.1 & 87.4 & 88.5 \\
\hline Instruments & 88.7 & 85.8 & 62.6 & 61.3 & 59.8 & 58.5 & 63.0 & 77.1 & 77.0 & 78.6 & 78.0 & 100.0 & 114.0 & 113.1 & 113.3 & 111.3 \\
\hline Other manufa & 93.8 & 84.4 & 72.4 & 71.8 & 71.9 & 77.2 & 87.4 & 92.4 & 100.1 & 87.9 & 85.6 & 100.0 & 88.8 & 84.9 & 80.4 & 86.2 \\
\hline
\end{tabular}

Source: OECD, DSTI (STAN Industrial Database), 1996. 
DSTI/DOC(98)1

Annex Table 29: Trade-Weighted Relative Unit Labour Cost per Industry - Total price competitiveness

Finland

\begin{tabular}{|c|c|c|c|c|c|c|c|c|c|c|c|c|c|c|c|c|}
\hline & 1979 & 1980 & 1981 & 1982 & 1983 & 1984 & 1985 & 1986 & 1987 & 1988 & 1989 & 1990 & 1991 & 1992 & 1993 & 1994 \\
\hline Total manufacturing & 83.8 & 84.5 & 87.6 & 91.5 & 89.6 & 92.1 & 93.3 & 90.5 & 88.2 & 90.6 & 95.4 & 100.0 & 96.8 & 75.1 & 59.8 & 62.1 \\
\hline Food, drink and tobacco & 77.9 & 82.8 & 83.6 & 88.5 & 83.5 & 87.5 & 88.3 & 91.3 & 89.1 & 93.1 & 97.6 & 100.0 & 93.9 & 75.7 & 60.7 & 63.3 \\
\hline Textiles, Footwear and leather & 76.7 & 79.7 & 82.0 & 87.6 & 88.5 & 90.4 & 91.4 & 87.9 & 89.2 & 95.4 & 101.3 & 100.0 & 96.5 & 73.0 & 63.4 & 65.1 \\
\hline Wood, cork and furniture & 86.8 & 88.1 & 92.7 & 95.0 & 89.7 & 96.4 & 98.1 & 95.6 & 92.3 & 90.0 & 94.4 & 100.0 & 98.7 & 73.1 & 59.0 & 62.8 \\
\hline Paper and printing & 91.1 & 88.5 & 90.8 & 95.7 & 89.8 & 90.0 & 90.8 & 91.0 & 91.9 & 91.0 & 96.1 & 100.0 & 94.0 & 75.0 & 62.4 & 64.9 \\
\hline Chemicals & 77.5 & 75.1 & 82.5 & 88.5 & 90.1 & 92.4 & 91.4 & 92.1 & 90.8 & 90.5 & 95.8 & 100.0 & 97.9 & 79.6 & 68.9 & 72.0 \\
\hline Industrial chemicals & 76.7 & 73.2 & 80.6 & 83.2 & 85.2 & 84.3 & 88.1 & 91.5 & 95.5 & 90.7 & 91.5 & 100.0 & 98.4 & 77.4 & 68.6 & 70.6 \\
\hline Pharmaceuticals & 64.2 & 52.7 & 56.1 & 64.9 & 73.0 & 83.9 & 85.3 & 83.5 & 83.5 & 83.5 & 87.7 & 100.0 & 100.4 & 94.8 & 76.4 & 88.1 \\
\hline Petroleum products & 54.4 & 46.1 & 50.2 & 54.5 & 58.4 & 67.5 & 63.8 & 75.0 & 68.5 & 86.4 & 113.3 & 100.0 & 91.9 & 73.5 & 67.5 & 61.1 \\
\hline Rubber and plastic products & 108.8 & 100.5 & 109.3 & 116.7 & 116.2 & 120.0 & 108.4 & 96.2 & 89.3 & 87.8 & 91.5 & 100.0 & 101.3 & 80.6 & 65.5 & 73.9 \\
\hline Stone, clay and glass & 75.1 & 73.3 & 77.1 & 77.9 & 80.8 & 94.3 & 91.2 & 90.0 & 85.4 & 91.5 & 95.1 & 100.0 & 94.7 & 75.6 & 61.5 & 64.9 \\
\hline Basic metals & 82.7 & 75.0 & 82.1 & 82.3 & 83.7 & 87.8 & 90.9 & 89.2 & 87.0 & 87.9 & 91.9 & 100.0 & 93.1 & 75.9 & 66.9 & 75.1 \\
\hline Ferrous metals & 75.2 & 69.1 & 75.7 & 75.1 & 78.0 & 85.8 & 89.6 & 86.8 & 83.7 & 88.4 & 94.2 & 100.0 & 90.0 & 71.3 & 61.7 & 70.1 \\
\hline Non-ferrous metals & 112.1 & 97.9 & 108.3 & 108.3 & 101.7 & 94.8 & 94.9 & 96.9 & 96.6 & 87.6 & 87.8 & 100.0 & 102.4 & 91.5 & 84.3 & 91.7 \\
\hline Fabricated metal products and machinery & 86.9 & 89.1 & 86.9 & 88.3 & 89.2 & 90.0 & 91.4 & 87.7 & 85.5 & 89.8 & 94.1 & 100.0 & 100.7 & 74.6 & 56.2 & 57.6 \\
\hline Fabricated metal products & 91.0 & 92.1 & 91.8 & 94.0 & 97.5 & 100.9 & 96.7 & 87.7 & 82.2 & 86.9 & 96.8 & 100.0 & 98.2 & 72.6 & 60.9 & 63.4 \\
\hline Non-electrical machinery & 93.5 & 95.8 & 86.3 & 87.6 & 94.6 & 95.2 & 90.8 & 92.1 & 90.6 & 92.8 & 95.7 & 100.0 & 109.0 & 83.4 & 63.2 & 66.2 \\
\hline Electrical machinery & 107.2 & 122.8 & 134.9 & 139.3 & 132.7 & 133.2 & 133.1 & 119.2 & 105.9 & 106.1 & 97.8 & 100.0 & 98.8 & 62.8 & 37.5 & 35.1 \\
\hline Shipbuilding & 57.0 & 86.0 & 84.7 & 77.4 & 60.7 & & 66.7 & 67.3 & 68.6 & 80.3 & 84.9 & 100.0 & 86.5 & 68.6 & 47.4 & 44.6 \\
\hline Other transport & 84.1 & 74.9 & 83.5 & 85.4 & 89.3 & 100.9 & 109.5 & 102.2 & 79.7 & 95.6 & 111.2 & 100.0 & 103.1 & 69.1 & 56.7 & 58.5 \\
\hline Motor vehicles & 66.4 & 70.2 & 73.8 & 70.8 & 73.0 & 78.9 & 87.9 & 85.5 & 88.6 & 87.9 & 98.7 & 100.0 & 101.1 & .. & .. & \\
\hline Aerospace & 39.8 & 28.6 & 28.3 & 26.8 & 34.6 & 44.0 & 41.6 & 58.0 & 64.1 & 72.8 & 83.2 & 100.0 & 96.9 & 80.0 & 85.7 & 73.0 \\
\hline Instruments & 104.9 & 98.7 & 83.9 & 82.4 & 76.1 & 67.9 & 79.2 & 75.7 & 72.2 & 83.6 & 85.3 & 100.0 & 102.9 & 83.8 & 63.0 & 70.1 \\
\hline Other manufacturing industries & 107.9 & 85.6 & 86.5 & 99.6 & 89.1 & 101.0 & 98.6 & 93.7 & 96.9 & 101.4 & 102.9 & 100.0 & 89.7 & 82.2 & 75.2 & 72.7 \\
\hline
\end{tabular}

Annex Table 30: Trade-Weighted Relative Unit Labour Cost per Industry - Competitiveness among exporters

Finland

\begin{tabular}{|c|c|c|c|c|c|c|c|c|c|c|c|c|c|c|c|c|}
\hline & 1979 & 1980 & 1981 & 1982 & 1983 & 1984 & 1985 & 1986 & 1987 & 1988 & 1989 & 1990 & 1991 & 1992 & 1993 & 1994 \\
\hline $\begin{array}{l}\text { Total manufacturing } \\
\end{array}$ & 83.0 & 84.5 & 86.6 & 87.1 & 82.8 & 84.4 & 86.2 & 85.6 & 85.6 & 88.8 & 93.3 & 100.0 & 96.4 & 74.6 & 56.4 & 58.4 \\
\hline Food, drink and tobacco & 72.5 & 78.5 & 80.5 & 83.0 & 75.9 & 78.0 & 82.3 & 86.3 & 85.1 & 90.9 & 96.0 & 100.0 & 93.2 & 74.7 & 56.7 & 60.4 \\
\hline Textiles, Footwear and leather & 74.7 & 79.7 & 83.2 & 87.4 & 86.4 & 86.5 & 88.4 & 85.7 & 86.9 & 93.9 & 100.2 & 100.0 & 96.7 & 73.4 & 61.5 & 63.4 \\
\hline Wood, cork and furniture & 82.3 & 87.7 & 91.5 & 90.7 & 83.7 & 88.4 & 91.5 & 92.2 & 90.0 & 89.1 & 91.9 & 100.0 & 99.5 & 74.1 & 57.7 & 61.2 \\
\hline Paper and printing & 91.6 & 91.8 & 93.0 & 94.1 & 85.3 & 84.7 & 86.9 & 89.5 & 91.5 & 91.4 & 94.7 & 100.0 & 93.3 & 75.0 & 60.1 & 63.1 \\
\hline Chemicals & 74.5 & 71.9 & 79.9 & 81.6 & 81.8 & 83.7 & 84.3 & 87.7 & 89.5 & 89.8 & 95.1 & 100.0 & 97.1 & 79.0 & 65.0 & 66.9 \\
\hline Industrial chemicals & 73.1 & 69.7 & 77.4 & 76.9 & 77.1 & 75.6 & 80.4 & 86.6 & 95.2 & 89.6 & 91.2 & 100.0 & 97.7 & 77.6 & 65.2 & 67.0 \\
\hline Pharmaceuticals & 64.0 & 48.6 & 50.3 & 56.2 & 63.6 & 75.0 & 77.5 & 80.4 & 81.3 & 82.6 & 85.6 & 100.0 & 99.2 & 92.7 & 72.8 & 81.4 \\
\hline Petroleum products & 49.3 & 48.1 & 47.1 & 49.1 & 50.5 & 58.4 & 57.3 & 68.7 & 70.9 & 91.0 & 114.0 & 100.0 & 92.1 & 81.7 & 71.7 & 64.7 \\
\hline Rubber and plastic products & 101.8 & 94.9 & 107.2 & 109.3 & 106.7 & 109.2 & 100.3 & 90.6 & 86.1 & 86.4 & 91.1 & 100.0 & 100.3 & 78.5 & 59.2 & 66.7 \\
\hline Stone, clay and glass & 75.6 & 75.7 & 80.3 & 74.3 & 75.0 & 85.3 & 87.5 & 87.3 & 83.4 & 90.4 & 93.3 & 100.0 & 94.9 & 76.4 & 59.2 & 62.7 \\
\hline Basic metals & 88.0 & 81.6 & 89.3 & 83.4 & 78.8 & 83.6 & 87.7 & 85.2 & 83.5 & 86.6 & 91.8 & 100.0 & 91.8 & 73.0 & 58.6 & 65.8 \\
\hline Ferrous metals & 79.0 & 76.5 & 83.2 & 77.1 & 74.1 & 82.8 & 86.5 & 80.8 & 79.3 & 85.0 & 92.8 & 100.0 & 87.3 & 66.4 & 51.9 & 58.5 \\
\hline Non-ferrous metals & 119.4 & 103.3 & 112.9 & 107.5 & 95.6 & 90.2 & 94.6 & 99.0 & 97.1 & 92.5 & 90.8 & 100.0 & 104.7 & 93.0 & 80.6 & 89.9 \\
\hline Fabricated metal products and machinery & 85.4 & 88.1 & 83.8 & 82.8 & 81.9 & 82.7 & 83.4 & 81.8 & 82.2 & 87.4 & 91.6 & 100.0 & 100.6 & 74.2 & 53.4 & 54.6 \\
\hline Fabricated metal products & 93.7 & 91.9 & 91.4 & 90.8 & 89.9 & 92.2 & 90.4 & 83.1 & 79.5 & 84.9 & 95.1 & 100.0 & 97.9 & 72.5 & 57.2 & 60.1 \\
\hline Non-electrical machinery & 87.5 & 91.2 & 80.8 & 79.3 & 83.6 & 83.9 & 82.2 & 86.1 & 86.8 & 89.6 & 92.7 & 100.0 & 109.7 & 83.2 & 59.7 & 62.0 \\
\hline Electrical machinery & 101.1 & 113.6 & 119.3 & 125.2 & 118.2 & 117.5 & 117.3 & 107.1 & 98.4 & 100.4 & 95.0 & 100.0 & 96.8 & 60.6 & 36.2 & 34.2 \\
\hline Shipbuilding & 49.1 & 70.3 & 72.6 & 69.7 & 61.5 & 67.7 & 67.6 & 67.1 & 65.3 & 80.9 & 85.9 & 100.0 & 85.0 & 68.7 & 55.9 & 52.4 \\
\hline Other transport & 57.9 & 60.9 & 65.1 & 65.0 & 71.2 & 79.1 & 86.8 & 82.8 & 71.1 & 84.8 & 100.8 & 100.0 & 102.6 & 66.2 & 55.1 & 51.6 \\
\hline Motor vehicles & 65.7 & 69.1 & 70.9 & 65.6 & 64.0 & 69.7 & 79.4 & 78.4 & 80.6 & 83.3 & 96.4 & 100.0 & 101.0 & 75.8 & 73.2 & 71.2 \\
\hline Aerospace & 41.0 & 30.2 & 30.0 & 31.8 & 40.2 & 44.2 & 47.4 & 52.4 & 63.0 & 70.5 & 81.5 & 100.0 & 101.1 & 83.6 & 85.7 & 75.7 \\
\hline Instruments & 104.0 & 96.4 & 82.9 & 80.3 & 75.6 & 71.4 & 81.1 & 75.9 & 72.9 & 83.2 & 86.9 & 100.0 & 102.8 & 83.5 & 59.1 & 66.7 \\
\hline Other manufact & 103.7 & 103.3 & 99.8 & 105.5 & 99.9 & 111.1 & 106.8 & 95.1 & 95.1 & 98.7 & 98.2 & 100.0 & 91.7 & 82.5 & 70.2 & 71.9 \\
\hline
\end{tabular}

Source: OECD, DSTI (STAN Industrial Database), 1996. 


\begin{tabular}{|c|c|c|c|c|c|c|c|c|c|c|c|c|c|c|c|c|}
\hline & 1979 & 1980 & 1981 & 1982 & 1983 & 1984 & 1985 & 1986 & 1987 & 1988 & 1989 & 1990 & 1991 & 1992 & 1993 & 1994 \\
\hline Total manufacturing & 100.1 & 104.3 & 102.3 & 99.2 & 97.9 & 98.7 & 102.1 & 106.1 & 105.2 & 99.6 & 96.7 & 100.0 & 98.4 & 99.4 & 101.2 & $\overline{101.5}$ \\
\hline Food, drink and tobacco & 87.4 & 97.4 & 99.8 & 92.5 & 98.9 & 97.0 & 100.1 & 106.5 & 103.8 & 101.5 & 98.3 & 100.0 & 93.1 & 98.7 & 103.9 & 99.2 \\
\hline Textiles, Footwear and leather & 96.4 & 99.8 & 98.9 & 93.8 & 94.2 & 93.6 & 97.9 & 103.3 & 106.7 & 105.6 & 101.3 & 100.0 & 98.3 & 97.4 & 104.1 & 100.5 \\
\hline Wood, cork and furniture & 106.8 & 108.6 & 110.4 & 107.6 & 108.8 & 109.6 & 111.2 & 114.1 & 109.0 & 104.4 & 100.6 & 100.0 & 99.0 & 102.7 & 109.3 & 102.9 \\
\hline Paper and printing & 91.7 & 93.2 & 93.5 & 90.6 & 93.4 & 95.8 & 95.5 & 102.2 & 105.9 & 99.8 & 96.1 & 100.0 & 103.7 & 109.9 & 119.5 & 116.2 \\
\hline Chemicals & 102.6 & 105.8 & 99.4 & 95.8 & 92.8 & 95.5 & 104.0 & 112.5 & 110.5 & 103.4 & 100.3 & 100.0 & 99.2 & 96.9 & 98.6 & 102.1 \\
\hline Industrial chemicals & 119.8 & 126.0 & 121.2 & 112.8 & 112.2 & 109.0 & 109.9 & 124.3 & 113.3 & 104.9 & 102.5 & 100.0 & 98.8 & 95.5 & 98.4 & 101.2 \\
\hline Pharmaceuticals & 168.2 & 155.4 & 151.7 & 138.4 & 113.6 & 102.9 & 100.6 & 106.2 & 109.1 & 97.6 & 94.3 & 100.0 & 97.7 & 95.2 & 100.1 & 95.8 \\
\hline Petroleum products & 96.6 & 88.7 & 75.9 & 71.0 & 64.5 & 72.4 & 117.4 & 143.0 & 139.5 & 129.9 & 109.4 & 100.0 & 79.0 & 49.8 & 52.6 & 53.9 \\
\hline Rubber and plastic products & 97.8 & 105.7 & 112.9 & 110.5 & 109.5 & 109.8 & 113.6 & 114.4 & 107.9 & 106.8 & 100.7 & 100.0 & 99.3 & 98.5 & 104.7 & 98.1 \\
\hline Stone, clay and glass & 108.4 & 104.9 & 105.4 & 100.9 & 104.8 & 101.0 & 102.1 & 104.8 & 106.0 & 101.8 & 99.1 & 100.0 & 96.1 & 97.3 & 101.8 & 100.3 \\
\hline Basic metals & 109.7 & 115.5 & 112.2 & 118.7 & 120.9 & 119.7 & 124.5 & 106.6 & 107.2 & 98.8 & 89.6 & 100.0 & 99.1 & 95.9 & 100.1 & 109.7 \\
\hline Ferrous metals & 105.9 & 106.2 & 107.0 & 119.2 & 125.6 & 126.2 & 131.2 & 112.2 & 107.5 & 98.7 & 90.3 & 100.0 & 99.6 & 95.4 & 100.5 & 107.7 \\
\hline Non-ferrous metals & 123.5 & 158.5 & 130.9 & 120.8 & 111.1 & 106.9 & 110.4 & 96.2 & 106.4 & 98.2 & 88.1 & 100.0 & 97.5 & 96.9 & 98.5 & 110.9 \\
\hline Fabricated metal products and machinery & 100.1 & 103.5 & 99.5 & 97.7 & 94.2 & 95.7 & 98.1 & 102.5 & 101.1 & 95.7 & 94.8 & 100.0 & 98.9 & 99.2 & 98.5 & 99.4 \\
\hline Fabricated metal products & 82.0 & 86.4 & 90.9 & 95.0 & 92.2 & 94.7 & 94.9 & 96.8 & 96.2 & 93.6 & 97.4 & 100.0 & 98.2 & 99.1 & 104.8 & 103.5 \\
\hline Non-electric & 110.3 & 114.5 & 108.3 & 102.1 & 102.9 & 101.4 & 102.4 & 110.9 & 108.6 & 100.5 & 101.3 & 100.0 & 98.6 & 99.2 & 93.3 & 87.5 \\
\hline Electrical machinery & 89.4 & 97.5 & 91.9 & 85.0 & 83.2 & 77.7 & 86.6 & 98.7 & 97.5 & 90.9 & 93.3 & 100.0 & 96.9 & 96.3 & 96.2 & 96.5 \\
\hline Shipbuilding & 159.1 & 149.6 & 106.7 & 118.4 & 84.8 & 113.4 & 107.9 & 123.6 & 107.1 & 132.2 & 78.6 & 100.0 & 89.2 & 91.9 & 128.2 & 88.8 \\
\hline Other transport & 103.0 & 114.1 & 105.9 & 106.1 & 119.1 & 113.8 & 120.7 & 138.4 & 114.7 & 107.8 & 110.5 & 100.0 & 95.9 & 95.5 & 83.2 & 93.5 \\
\hline Motor vehicles & 121.6 & 122.0 & 125.1 & 124.2 & 120.8 & 126.8 & 127.6 & 116.0 & 108.1 & 99.5 & 94.7 & 100.0 & 102.8 & 109.4 & 107.0 & 129.4 \\
\hline Aerospace & 101.9 & 100.7 & 64.6 & 71.7 & 62.9 & 71.7 & 65.3 & 84.0 & 103.8 & 93.8 & 87.8 & 100.0 & 101.0 & 94.1 & 101.4 & 90.3 \\
\hline Instruments & 91.4 & 101.3 & 89.8 & 79.0 & 72.2 & 66.2 & 66.5 & 78.4 & 88.7 & 92.9 & 87.8 & 100.0 & 99.2 & 98.3 & 90.8 & 86.4 \\
\hline Other manufactı & 79.8 & 88.2 & 89.0 & 80.4 & 65.8 & 66.9 & 81.2 & 95.6 & 103.4 & 93.9 & 90.0 & 100.0 & 95.0 & 105.2 & 114.6 & 2 \\
\hline
\end{tabular}

Annex Table 32: Trade-Weighted Relative Unit Labour Cost per Industry - Competitiveness among exporters

France

\begin{tabular}{|c|c|c|c|c|c|c|c|c|c|c|c|c|c|c|c|c|}
\hline & 1979 & 1980 & 1981 & 1982 & 1983 & 1984 & 1985 & 1986 & 1987 & 1988 & 1989 & 1990 & 1991 & 1992 & 1993 & $\overline{1994}$ \\
\hline Total manufacturing & 102.2 & 106.3 & 99.4 & 94.4 & 91.6 & 90.7 & 94.1 & 100.2 & 102.4 & 96.0 & 92.8 & 100.0 & 97.6 & 99.5 & 97.6 & 96.3 \\
\hline Food, drink and tobacco & 89.4 & 100.7 & 98.9 & 88.5 & 91.1 & 87.7 & 92.0 & 101.7 & 101.6 & 99.7 & 95.3 & 100.0 & 93.0 & 99.8 & 103.2 & 99.3 \\
\hline Textiles, Footwear and leather & 99.9 & 103.0 & 97.8 & 91.3 & 92.0 & 91.1 & 96.3 & 102.4 & 105.8 & 104.2 & 99.7 & 100.0 & 98.7 & 97.2 & 100.7 & 97.6 \\
\hline Wood, cork and furniture & 111.3 & 112.8 & 108.5 & 100.4 & 100.7 & 100.2 & 102.8 & 111.3 & 110.3 & 102.9 & 97.3 & 100.0 & 99.2 & 105.0 & 110.8 & 106.1 \\
\hline Paper and printing & 101.3 & 102.8 & 97.0 & 89.0 & 89.3 & 88.7 & 89.5 & 101.6 & 107.8 & 99.5 & 93.4 & 100.0 & 103.8 & 112.2 & 119.8 & 115.6 \\
\hline Chemicals & 101.8 & 103.5 & 94.7 & 89.1 & 86.2 & 87.4 & 96.1 & 106.3 & 108.8 & 100.8 & 97.2 & 100.0 & 98.7 & 97.3 & 96.4 & 97.6 \\
\hline Industrial chemicals & 118.0 & 122.1 & 114.9 & 105.7 & 105.3 & 101.6 & 104.0 & 117.8 & 112.4 & 104.4 & 101.0 & 100.0 & 99.1 & 96.4 & 96.9 & 98.8 \\
\hline Pharmaceuticals & 172.7 & 160.4 & 156.0 & 139.8 & 116.6 & 104.7 & 100.9 & 106.3 & 109.6 & 98.3 & 93.9 & 100.0 & 98.1 & 95.1 & 98.8 & 91.8 \\
\hline Petroleum products & 86.3 & 82.7 & 66.1 & 63.3 & 59.5 & 66.7 & 102.2 & 128.1 & 138.1 & 128.0 & 105.3 & 100.0 & 81.1 & 51.9 & 53.9 & 58.1 \\
\hline Rubber and plastic products & 102.0 & 108.9 & 113.0 & 107.2 & 105.7 & 102.9 & 105.4 & 108.0 & 105.4 & 100.9 & 96.3 & 100.0 & 98.5 & 99.0 & 102.3 & 94.4 \\
\hline Stone, clay and glass & 110.2 & 107.3 & 103.4 & 97.0 & 101.8 & 96.5 & 99.0 & 102.6 & 104.7 & 98.8 & 96.4 & 100.0 & 95.2 & 96.7 & 97.8 & 95.8 \\
\hline Basic metals & 110.0 & 115.3 & 108.5 & 113.6 & 112.6 & 114.6 & 117.6 & 99.7 & 103.1 & 95.8 & 87.3 & 100.0 & 98.2 & 94.5 & 93.8 & 101.4 \\
\hline Ferrous metals & 106.3 & 107.6 & 105.5 & 117.6 & 118.8 & 123.1 & 126.5 & 104.9 & 102.9 & 94.9 & 87.7 & 100.0 & 97.8 & 92.3 & 90.7 & 95.3 \\
\hline Non-ferrous metals & 119.7 & 152.7 & 117.8 & 106.9 & 100.7 & 99.8 & 102.1 & 91.1 & 104.3 & 97.9 & 86.6 & 100.0 & 99.1 & 99.1 & 100.3 & 113.0 \\
\hline Fabricated metal products and machinery & 96.4 & 101.8 & 93.0 & 90.4 & 86.0 & 86.1 & 89.0 & 94.8 & 96.2 & 90.9 & 90.2 & 100.0 & 97.9 & 99.0 & 95.1 & 94.2 \\
\hline Fabricated metal products & 85.5 & 90.1 & 89.6 & 90.8 & 86.6 & 86.9 & 88.7 & 94.3 & 95.5 & 91.6 & 93.7 & 100.0 & 97.6 & 99.6 & 102.8 & 100.6 \\
\hline Non-electrical machinery & 100.1 & 106.6 & 97.0 & 91.1 & 93.7 & 91.8 & 95.3 & 105.5 & 104.5 & 97.1 & 97.4 & 100.0 & 98.2 & 99.5 & 89.2 & 82.2 \\
\hline Electrical machinery & 74.2 & 84.7 & 77.0 & 73.2 & 73.8 & 69.1 & 78.5 & 90.1 & 90.6 & 85.8 & 88.7 & 100.0 & 96.5 & 96.1 & 93.8 & 92.4 \\
\hline Shipbuilding & 141.6 & 147.1 & 108.7 & 124.9 & 88.9 & 113.3 & 126.4 & 118.4 & 111.2 & 124.4 & 77.7 & 100.0 & 89.9 & 84.6 & 106.9 & 86.4 \\
\hline Other transport & 69.3 & 89.6 & 82.0 & 82.8 & 95.7 & 97.6 & 107.3 & 109.5 & 96.1 & 89.6 & 99.4 & 100.0 & 96.7 & 92.3 & 73.6 & 71.3 \\
\hline Motor vehicles & 120.4 & 127.7 & 125.7 & 124.2 & 115.4 & 116.9 & 115.4 & 105.1 & 102.5 & 93.9 & 89.8 & 100.0 & 99.1 & 105.6 & 102.2 & 117.5 \\
\hline Aerospace & 102.4 & 97.8 & 68.2 & 74.0 & 66.9 & 79.4 & 72.4 & 88.3 & 100.6 & 88.9 & 87.6 & 100.0 & 102.3 & 94.7 & 105.0 & 93.7 \\
\hline Instruments & 89.6 & 99.8 & 90.7 & 79.7 & 75.3 & 72.0 & 73.3 & 81.5 & 89.3 & 91.2 & 89.3 & 100.0 & 98.8 & 97.0 & 87.6 & 84.4 \\
\hline Other manufa & 73.6 & 91.6 & 90.9 & 82.0 & 71.2 & 72.3 & 86.2 & 99.5 & 104.4 & 92.9 & 86.6 & 100.0 & 97.8 & 106.9 & 112.2 & 124.3 \\
\hline
\end{tabular}

Source: OECD, DSTI (STAN Industrial Database), 1996. 
DSTI/DOC(98)1

Annex Table 33: Trade-Weighted Relative Unit Labour Cost per Industry - Total price competitiveness

Western Germany

\begin{tabular}{|c|c|c|c|c|c|c|c|c|c|c|c|c|c|c|c|c|}
\hline & 1979 & 1980 & 1981 & 1982 & 1983 & 1984 & 1985 & 1986 & 1987 & 1988 & 1989 & 1990 & 1991 & 1992 & 1993 & 1994 \\
\hline Total manufacturing & 84.1 & 84.8 & 79.6 & 83.4 & 84.9 & 82.5 & 80.9 & 89.3 & 98.6 & 98.4 & 97.1 & 100.0 & 96.8 & 103.2 & 108.7 & 107.8 \\
\hline Food, drink and tobacco & 86.7 & 88.9 & 87.1 & 94.7 & 91.3 & 85.3 & 86.3 & 94.9 & 95.7 & 95.7 & 101.7 & 100.0 & 103.6 & 113.3 & 115.4 & 120.5 \\
\hline Textiles, Footwear and leather & 105.0 & 104.8 & 100.5 & 102.0 & 101.5 & 96.5 & 94.5 & 103.8 & 107.2 & 105.5 & 100.6 & 100.0 & 98.4 & 102.7 & 114.3 & 122.8 \\
\hline Wood, cork and furniture & 78.4 & 79.6 & 77.6 & 84.9 & 90.1 & 89.2 & 87.2 & 95.2 & 97.6 & 101.2 & 100.4 & 100.0 & 97.1 & 97.9 & 107.2 & 105.0 \\
\hline Paper and printing & 101.9 & 101.6 & 96.4 & 96.3 & 96.0 & 89.5 & 89.3 & 96.4 & 98.2 & 102.5 & 101.4 & 100.0 & 98.0 & 104.6 & 117.6 & 119.5 \\
\hline Chemicals & 70.0 & 67.9 & 68.8 & 71.4 & 75.7 & 75.9 & 76.4 & 79.0 & 96.1 & 95.3 & 96.2 & 100.0 & 101.1 & 106.4 & 108.1 & 107.4 \\
\hline Industrial chemicals & 68.3 & 65.2 & 64.9 & 67.7 & 68.7 & 68.5 & 70.4 & 73.4 & 92.3 & 90.9 & 93.7 & 100.0 & 102.5 & 110.2 & 112.8 & 106.8 \\
\hline Pharmaceuticals & 81.4 & 73.1 & 69.2 & 67.4 & 80.7 & 83.0 & 79.6 & 85.5 & 106.3 & 105.4 & 100.4 & 100.0 & 89.2 & 99.5 & 107.1 & 107.3 \\
\hline Petroleum products & 59.7 & 66.3 & 59.0 & 60.6 & 74.8 & 79.6 & 81.6 & 86.0 & 100.9 & 99.0 & 98.9 & 100.0 & 98.5 & 94.4 & 105.5 & 107.6 \\
\hline Rubber and plastic products & 89.8 & 85.9 & 84.8 & 86.5 & 84.2 & 79.9 & 84.9 & 85.7 & 100.7 & 100.9 & 97.4 & 100.0 & 100.9 & 107.6 & 109.4 & 113.9 \\
\hline Stone, clay and glass & 93.2 & 94.5 & 90.1 & 91.0 & 94.4 & 93.1 & 93.2 & 96.5 & 103.5 & 105.9 & 99.9 & 100.0 & 95.6 & 100.5 & 109.4 & 112.7 \\
\hline Basic metals & 86.9 & 85.3 & 77.5 & 82.8 & 81.2 & 85.1 & 81.2 & 92.1 & 98.9 & 104.6 & 101.9 & 100.0 & 97.6 & 101.0 & 111.5 & 105.4 \\
\hline Ferrous metals & 89.3 & 90.7 & 82.9 & 88.4 & 81.0 & 85.6 & 81.2 & 90.9 & 99.4 & 105.9 & 102.5 & 100.0 & 94.9 & 99.5 & 109.4 & 103.0 \\
\hline Non-ferrous metals & 76.6 & 73.1 & 63.9 & 66.0 & 81.8 & 85.9 & 83.6 & 95.3 & 98.6 & 103.5 & 102.4 & 100.0 & 104.7 & 105.3 & 116.9 & 113.0 \\
\hline Fabricated metal products and machinery & 82.1 & 84.0 & 73.7 & 76.8 & 80.0 & 76.9 & 74.6 & 86.2 & 96.1 & 96.2 & 94.5 & 100.0 & 94.2 & 102.3 & 109.2 & 107.9 \\
\hline Fabricated metal products & 85.1 & 86.7 & 83.7 & 90.6 & 90.4 & 92.1 & 91.7 & 97.2 & 102.9 & 101.8 & 99.9 & 100.0 & 96.5 & 103.4 & 111.7 & 122.3 \\
\hline Non-electrical machinery & 76.3 & 78.3 & 67.9 & 69.8 & 77.3 & 76.2 & 75.8 & 87.2 & 97.8 & 97.8 & 93.8 & 100.0 & 96.2 & 106.2 & 112.5 & 116.1 \\
\hline Electrical machinery & 77.8 & 75.8 & 67.1 & 68.8 & 74.0 & 72.5 & 73.0 & 84.9 & 93.7 & 96.7 & 94.4 & 100.0 & 96.6 & 100.7 & 119.2 & 113.3 \\
\hline Shipbuilding & 44.5 & 50.2 & 56.0 & 53.1 & 53.5 & 50.0 & 51.2 & 69.4 & 64.9 & 88.2 & 96.3 & 100.0 & 100.3 & 114.3 & 130.3 & 124.3 \\
\hline Other transport & 109.2 & 117.0 & 101.3 & 106.2 & 103.8 & 92.6 & 82.6 & 96.5 & 108.7 & 104.2 & 97.9 & 100.0 & 93.4 & 96.8 & 180.0 & 159.1 \\
\hline Motor vehicles & 79.9 & 84.6 & 72.3 & 74.1 & 78.3 & 77.8 & 73.7 & 86.1 & 102.5 & 103.2 & 98.6 & 100.0 & 88.9 & 97.3 & 103.2 & 88.4 \\
\hline Aerospace & & & & & & & & & & & & & .. & .. & .. & .. \\
\hline Instruments & 77.5 & 86.4 & 72.4 & 73.5 & 71.1 & 67.8 & 68.2 & 78.0 & 92.8 & 90.3 & 91.7 & 100.0 & 95.1 & 99.6 & 93.1 & 95.9 \\
\hline Other manufacturing industries & 92.9 & 95.8 & 82.5 & 79.4 & 80.4 & 84.8 & 86.5 & 86.4 & 93.8 & 93.2 & 94.1 & 100.0 & 98.1 & 96.0 & 94.7 & 94.2 \\
\hline
\end{tabular}

Annex Table 34: Trade-Weighted Relative Unit Labour Cost per Industry - Competitiveness among exporters

Western Germany

\begin{tabular}{|c|c|c|c|c|c|c|c|c|c|c|c|c|c|c|c|c|}
\hline & 1979 & 1980 & 1981 & 1982 & 1983 & 1984 & 1985 & 1986 & 1987 & 1988 & 1989 & 1990 & 1991 & 1992 & 1993 & 1994 \\
\hline Total manufacturing & 87.3 & 87.1 & 78.1 & 80.6 & 80.6 & 77.1 & 76.2 & 86.4 & 98.0 & 96.5 & 94.2 & 100.0 & 96.0 & 103.3 & 105.8 & 103.7 \\
\hline Food, drink and tobacco & 92.6 & 93.1 & 86.3 & 92.8 & 88.2 & 80.5 & 82.5 & 94.3 & 99.8 & 98.5 & 100.2 & 100.0 & 102.3 & 112.6 & 113.6 & 118.9 \\
\hline Textiles, Footwear and leather & 100.4 & 99.9 & 94.2 & 96.8 & 97.5 & 92.1 & 90.8 & 100.8 & 105.0 & 103.0 & 99.0 & 100.0 & 98.1 & 101.9 & 110.8 & 119.3 \\
\hline Wood, cork and furniture & 79.5 & 80.7 & 75.7 & 80.8 & 84.6 & 83.3 & 82.7 & 95.6 & 100.2 & 101.1 & 97.9 & 100.0 & 96.2 & 99.1 & 107.1 & 105.5 \\
\hline Paper and printing & 107.5 & 106.0 & 96.6 & 94.4 & 93.3 & 85.1 & 84.5 & 96.1 & 100.4 & 102.6 & 99.2 & 100.0 & 97.3 & 105.5 & 116.7 & 117.1 \\
\hline Chemicals & 72.7 & 69.3 & 67.2 & 68.6 & 71.7 & 69.5 & 71.1 & 76.6 & 96.9 & 94.4 & 93.9 & 100.0 & 100.4 & 107.2 & 107.1 & 104.4 \\
\hline Industrial chemicals & 71.3 & 66.6 & 63.8 & 66.0 & 67.0 & 64.9 & 67.3 & 72.2 & 93.7 & 91.7 & 92.8 & 100.0 & 102.4 & 110.9 & 110.5 & 105.0 \\
\hline Pharmaceuticals & 83.2 & 73.4 & 67.8 & 67.4 & 77.6 & 78.2 & 74.6 & 83.8 & 106.9 & 105.5 & 99.0 & 100.0 & 89.4 & 100.1 & 108.5 & 105.5 \\
\hline Petroleum products & 67.4 & 70.3 & 66.8 & 68.7 & 77.2 & 81.1 & 76.7 & 83.5 & 110.5 & 102.6 & 99.3 & 100.0 & 98.6 & 97.4 & 108.5 & 104.1 \\
\hline Rubber and plastic products & 93.6 & 88.3 & 85.3 & 85.9 & 83.2 & 76.4 & 81.6 & 84.2 & 100.8 & 98.9 & 94.7 & 100.0 & 100.5 & 109.4 & 110.8 & 114.2 \\
\hline Stone, clay and glass & 93.5 & 94.2 & 87.8 & 88.7 & 92.4 & 88.8 & 90.2 & 95.0 & 103.0 & 103.6 & 97.4 & 100.0 & 94.5 & 100.2 & 105.4 & 107.7 \\
\hline Basic metals & 93.1 & 92.7 & 82.9 & 86.9 & 81.1 & 86.0 & 82.6 & 89.7 & 98.9 & 102.3 & 98.4 & 100.0 & 95.9 & 97.8 & 101.1 & 96.1 \\
\hline Ferrous metals & 95.2 & 98.8 & 89.0 & 94.1 & 82.1 & 88.7 & 84.2 & 88.1 & 98.6 & 101.8 & 98.5 & 100.0 & 92.3 & 94.0 & 95.6 & 89.8 \\
\hline Non-ferrous metals & 81.9 & 80.7 & 67.1 & 67.8 & 79.2 & 83.6 & 82.8 & 94.1 & 100.5 & 105.2 & 99.5 & 100.0 & 105.8 & 106.0 & 114.5 & 112.4 \\
\hline Fabricated metal products and machinery & 81.1 & 83.7 & 71.1 & 72.9 & 74.3 & 71.4 & 70.2 & 82.2 & 93.9 & 93.5 & 91.4 & 100.0 & 93.6 & 102.4 & 106.3 & 104.2 \\
\hline Fabricated metal products & 86.9 & 86.7 & 80.9 & 86.9 & 86.4 & 84.6 & 86.2 & 94.9 & 102.6 & 100.0 & 96.7 & 100.0 & 95.2 & 103.8 & 110.8 & 120.1 \\
\hline Non-electrical machinery & 71.2 & 74.8 & 64.0 & 65.1 & 72.7 & 72.3 & 73.0 & 85.3 & 95.7 & 95.2 & 91.5 & 100.0 & 95.9 & 105.9 & 105.6 & 105.9 \\
\hline Electrical machinery & 64.3 & 66.4 & 58.1 & 60.1 & 66.0 & 65.4 & 66.5 & 78.4 & 87.5 & 91.1 & 89.8 & 100.0 & 95.8 & 100.6 & 115.6 & 108.4 \\
\hline Shipbuilding & 45.5 & 59.3 & 58.9 & 56.1 & 54.6 & 51.5 & 53.9 & 69.6 & 63.2 & 80.4 & 92.1 & 100.0 & 98.7 & 116.3 & 124.7 & 115.4 \\
\hline Other transport & 75.1 & 93.0 & 80.3 & 85.3 & 85.2 & 84.1 & 76.3 & 81.0 & 97.2 & 92.9 & 89.5 & 100.0 & 91.9 & 91.7 & 165.7 & 144.6 \\
\hline Motor vehicles & 81.1 & 88.8 & 74.8 & 76.5 & 78.0 & 75.7 & 71.1 & 83.5 & 100.3 & 100.6 & 95.8 & 100.0 & 88.2 & 96.9 & 102.0 & 87.8 \\
\hline Aerospace & 92.7 & 86.7 & 54.4 & 61.9 & 64.0 & 58.3 & 58.2 & 90.2 & 102.5 & 102.7 & 97.1 & 100.0 & 92.5 & 109.8 & 111.1 & 106.0 \\
\hline Instruments & 79.6 & 87.1 & 75.3 & 75.1 & 75.2 & 75.0 & 75.5 & 82.3 & 93.9 & 89.2 & 93.1 & 100.0 & 95.0 & 98.2 & 90.2 & 93.5 \\
\hline & & & & 836 & 901 & 926 & 950 & 924 & 958 & 932 & & & & & & \\
\hline
\end{tabular}

Source: OECD, DSTI (STAN Industrial Database), 1996. 
Annex Table 35: Trade-Weighted Relative Unit Labour Cost per Industry - Total price competitiveness

Greece

\begin{tabular}{|c|c|c|c|c|c|c|c|c|c|c|c|c|c|c|c|c|}
\hline & 1979 & 1980 & 1981 & 1982 & 1983 & 1984 & 1985 & 1986 & 1987 & 1988 & 1989 & 1990 & 1991 & 1992 & 1993 & 1994 \\
\hline Total manufacturing & 85.9 & 82.6 & 88.1 & 99.9 & 96.2 & 100.5 & 95.3 & 79.9 & 80.1 & 85.4 & 93.5 & 100.0 & 96.4 & 98.4 & 103.8 & 112.3 \\
\hline Food, drink and tobacco & 75.8 & 78.2 & 85.2 & 99.8 & 94.7 & 97.0 & 92.5 & 82.7 & 78.7 & 81.6 & 89.0 & 100.0 & 94.1 & 95.8 & 96.9 & 100.1 \\
\hline Textiles, Footwear and leather & 73.6 & 73.5 & 83.0 & 103.8 & 99.2 & 102.7 & 99.1 & 82.9 & 83.7 & 90.0 & 101.1 & 100.0 & 97.7 & 100.2 & 107.0 & 109.7 \\
\hline Wood, cork and furniture & 64.1 & 68.4 & 60.8 & 67.9 & 89.5 & 97.8 & 86.1 & 78.4 & 91.3 & 88.9 & 92.6 & 100.0 & 102.3 & 112.2 & 126.5 & 143.9 \\
\hline Paper and printing & 108.4 & 108.2 & 108.6 & 112.8 & 105.9 & 105.5 & 90.8 & 74.9 & 67.8 & 79.3 & 86.1 & 100.0 & 95.0 & 101.4 & 110.1 & 114.6 \\
\hline Chemicals & 94.7 & 90.4 & 101.9 & 112.9 & 108.5 & 111.0 & 104.9 & 89.6 & 87.2 & 85.7 & 92.2 & 100.0 & 104.0 & 109.1 & 112.7 & 124.1 \\
\hline Industrial chemicals & 81.6 & 76.8 & 88.0 & 100.7 & 96.3 & 101.0 & 98.5 & 83.8 & 79.6 & 83.3 & 94.0 & 100.0 & 102.0 & 103.9 & 114.4 & 125.1 \\
\hline Pharmaceuticals & 105.8 & 103.9 & 116.0 & 132.4 & 118.5 & 108.2 & 98.4 & 94.7 & 84.8 & 85.8 & 90.5 & 100.0 & 114.8 & 120.5 & 110.6 & 124.7 \\
\hline Petroleum products & 79.2 & 71.3 & 81.7 & 84.8 & 90.6 & 97.3 & 96.8 & 67.2 & 67.6 & 70.0 & 88.5 & 100.0 & 108.9 & 120.5 & 110.1 & 120.9 \\
\hline Rubber and plastic products & 122.2 & 126.8 & 131.8 & 143.9 & 128.6 & 134.8 & 123.8 & 110.7 & 116.9 & 96.1 & 89.5 & 100.0 & 97.2 & 107.7 & 111.8 & 123.2 \\
\hline Stone, clay and glass & 94.2 & 85.5 & 93.2 & 103.8 & 101.4 & 105.6 & 103.1 & 83.8 & 84.5 & 90.5 & 98.5 & 100.0 & 108.0 & 115.2 & 123.9 & 128.7 \\
\hline Basic metals & 72.9 & 73.6 & 76.7 & 88.3 & 82.4 & 94.8 & 88.0 & 75.6 & 76.1 & 81.5 & 95.2 & 100.0 & 91.3 & 92.2 & 98.6 & 103.1 \\
\hline Ferrous metals & 87.1 & 84.5 & 85.9 & 82.6 & 82.6 & 89.0 & 84.9 & 78.3 & 78.5 & 82.5 & 89.4 & 100.0 & 96.0 & 97.7 & 107.1 & 116.3 \\
\hline Non-ferrous metals & 64.1 & 68.2 & 70.1 & 100.8 & 87.4 & 104.1 & 96.1 & 79.1 & 77.2 & 79.8 & 99.5 & 100.0 & 85.8 & 86.8 & 87.2 & 87.3 \\
\hline Fabricated metal products and machinery & 85.3 & 80.8 & 82.2 & 86.3 & 80.5 & 88.2 & 87.2 & 69.1 & 74.9 & 80.8 & 89.5 & 100.0 & 91.5 & 88.2 & 102.4 & 116.8 \\
\hline Fabricated metal products & 75.8 & 71.9 & 74.9 & 83.2 & 75.2 & 83.0 & 84.7 & 68.5 & 74.2 & 70.2 & 81.3 & 100.0 & 91.9 & 87.0 & 87.7 & 92.7 \\
\hline Non-electrical machinery & 69.0 & 72.3 & 69.1 & 75.5 & 74.5 & 86.4 & 78.9 & 69.9 & 117.5 & 117.0 & 105.0 & 100.0 & 97.3 & 89.6 & 86.7 & 89.6 \\
\hline Electrical machinery & 81.6 & 76.7 & 77.4 & 96.4 & 98.6 & 117.8 & 96.9 & 79.3 & 92.1 & 98.0 & 98.4 & 100.0 & 102.1 & 111.1 & 107.6 & 119.9 \\
\hline Shipbuilding & 42.2 & 57.3 & 55.2 & 46.9 & 43.4 & 61.7 & 99.0 & 43.5 & 43.0 & 77.2 & 88.5 & 100.0 & 85.2 & 93.5 & 87.6 & 86.9 \\
\hline Other transport & 94.9 & 86.5 & 84.0 & 72.9 & 69.1 & 87.9 & 84.0 & 73.0 & 80.1 & 86.2 & 92.0 & 100.0 & 91.4 & 83.3 & 67.9 & 106.2 \\
\hline Motor vehicles & 89.6 & 83.0 & 84.6 & 82.6 & 90.7 & 88.9 & 88.3 & 82.0 & 70.5 & 79.1 & 103.8 & 100.0 & 99.5 & 72.2 & 117.0 & 127.0 \\
\hline Aerospace & .. & .. & .. & .. & .. & .. & .. & .. & .. & .. & .. & .. & .. & .. & .. & .. \\
\hline Instruments & & & & & & & .. & .. & .. & .. & .. & .. & .. & .. & & \\
\hline Other manufacturing industries & 95.9 & 76.4 & 80.5 & 96.5 & 80.5 & 96.1 & 87.7 & 74.6 & 75.5 & 105.5 & 126.0 & 100.0 & 73.5 & 124.3 & 183.9 & 228.3 \\
\hline
\end{tabular}

Annex Table 36: Trade-Weighted Relative Unit Labour Cost per Industry - Competitiveness among exporters

Greece

\begin{tabular}{|c|c|c|c|c|c|c|c|c|c|c|c|c|c|c|c|c|}
\hline & 1979 & 1980 & 1981 & 1982 & 1983 & 1984 & 1985 & 1986 & 1987 & 1988 & 1989 & 1990 & 1991 & 1992 & 1993 & $\overline{1994}$ \\
\hline Total manufacturing & 83.3 & 80.8 & 83.7 & 94.7 & 90.3 & 92.7 & 87.2 & 75.8 & 78.6 & 82.8 & 90.4 & 100.0 & 95.7 & 98.2 & 100.3 & $\overline{105.4}$ \\
\hline Food, drink and tobacco & 78.5 & 80.7 & 83.0 & 98.2 & 90.4 & 89.6 & 85.4 & 81.0 & 80.6 & 83.2 & 88.2 & 100.0 & 94.3 & 95.9 & 95.7 & 99.2 \\
\hline Textiles, Footwear and leather & 73.4 & 74.1 & 82.5 & 104.4 & 99.8 & 101.3 & 98.2 & 82.9 & 84.3 & 90.1 & 100.6 & 100.0 & 97.0 & 99.3 & 105.9 & 108.4 \\
\hline Wood, cork and furniture & 60.4 & 66.1 & 60.0 & 65.3 & 82.2 & 90.3 & 83.4 & 76.8 & 89.5 & 87.3 & 90.4 & 100.0 & 102.5 & 112.5 & 117.4 & 132.0 \\
\hline Paper and printing & 110.9 & 108.1 & 108.1 & 110.3 & 100.5 & 97.5 & 84.5 & 72.9 & 68.1 & 78.4 & 84.1 & 100.0 & 94.4 & 101.3 & 106.0 & 108.8 \\
\hline Chemicals & 98.9 & 92.0 & 98.5 & 108.4 & 102.4 & 102.5 & 95.6 & 84.9 & 85.3 & 82.7 & 89.0 & 100.0 & 103.4 & 109.5 & 108.7 & 117.0 \\
\hline Industrial chemicals & 83.9 & 77.2 & 83.9 & 93.8 & 90.4 & 91.6 & 89.0 & 78.7 & 79.6 & 83.1 & 92.6 & 100.0 & 102.3 & 105.1 & 109.8 & 118.7 \\
\hline Pharmaceuticals & 106.3 & 104.1 & 113.3 & 127.5 & 114.8 & 103.3 & 97.0 & 92.6 & 84.5 & 85.4 & 90.2 & 100.0 & 114.6 & 120.6 & 109.8 & 117.6 \\
\hline Petroleum products & 70.6 & 63.1 & 61.8 & 70.4 & 71.1 & 80.1 & 76.0 & 63.0 & 66.5 & 68.8 & 85.0 & 100.0 & 106.6 & 109.4 & 106.0 & 115.3 \\
\hline Rubber and plastic products & 122.8 & 127.4 & 129.0 & 136.7 & 124.8 & 125.3 & 113.1 & 103.7 & 112.9 & 89.3 & 85.8 & 100.0 & 97.1 & 109.0 & 108.2 & 116.2 \\
\hline Stone, clay and glass & 88.2 & 82.2 & 86.8 & 98.1 & 97.6 & 99.4 & 97.9 & 81.8 & 82.7 & 86.9 & 96.6 & 100.0 & 107.5 & 114.3 & 114.7 & 117.7 \\
\hline Basic metals & 71.4 & 71.6 & 76.1 & 89.7 & 82.0 & 94.1 & 88.9 & 72.9 & 74.3 & 80.0 & 92.2 & 100.0 & 91.5 & 91.2 & 90.0 & 93.4 \\
\hline Ferrous metals & 84.6 & 84.2 & 88.1 & 88.3 & 84.4 & 92.5 & 90.3 & 76.4 & 76.5 & 80.8 & 87.6 & 100.0 & 96.4 & 96.0 & 93.5 & 100.6 \\
\hline Non-ferrous metals & 65.9 & 65.7 & 67.1 & 96.9 & 83.0 & 98.1 & 87.9 & 73.8 & 75.4 & 80.2 & 95.8 & 100.0 & 87.4 & 88.8 & 88.4 & 88.0 \\
\hline Fabricated metal products and machinery & 83.1 & 77.7 & 76.6 & 78.7 & 74.6 & 81.6 & 78.0 & 64.3 & 72.1 & 77.3 & 85.9 & 100.0 & 90.9 & 88.4 & 100.0 & 110.6 \\
\hline Fabricated metal products & 73.5 & 70.1 & 71.5 & 78.3 & 70.6 & 75.6 & 78.6 & 66.1 & 72.3 & 67.7 & 78.0 & 100.0 & 90.8 & 85.9 & 83.3 & 86.2 \\
\hline Non-electrical machinery & 60.4 & 65.6 & 60.9 & 64.1 & 65.6 & 78.1 & 69.9 & 65.3 & 111.1 & 112.3 & 102.1 & 100.0 & 97.1 & 89.0 & 83.2 & 84.6 \\
\hline Electrical machinery & 65.2 & 64.8 & 64.4 & 81.3 & 81.8 & 97.4 & 81.9 & 71.1 & 84.7 & 91.2 & 92.5 & 100.0 & 101.3 & 111.0 & 105.6 & 113.1 \\
\hline Shipbuilding & 53.2 & 60.1 & 66.4 & 62.1 & 53.0 & 72.3 & 111.0 & 56.0 & 55.3 & 68.6 & 84.1 & 100.0 & 87.0 & 98.1 & 91.8 & 87.6 \\
\hline Other transport & 55.1 & 52.4 & 57.6 & 67.8 & 69.5 & 82.0 & 77.7 & 61.1 & 71.9 & 75.9 & 82.3 & 100.0 & 88.0 & 79.6 & 79.3 & 85.5 \\
\hline Motor vehicles & 89.2 & 77.7 & 78.7 & 77.3 & 86.6 & 83.1 & 79.0 & 77.0 & 70.3 & 79.4 & 101.6 & 100.0 & 97.9 & 71.9 & 115.4 & 119.3 \\
\hline Aerospace & 100.5 & 83.5 & 75.1 & 81.9 & 77.2 & 82.5 & 71.8 & 65.7 & 79.9 & 84.8 & 89.0 & 100.0 & 90.2 & 87.4 & 98.9 & 112.1 \\
\hline Instruments & 47.8 & 39.0 & 30.8 & 45.6 & 43.4 & 38.2 & 45.3 & 46.9 & 25.2 & 57.2 & 105.5 & 100.0 & 85.1 & 86.5 & 106.2 & 138.9 \\
\hline Other manufa & 79.8 & 73.0 & 77.4 & 92.2 & 81.3 & 94.3 & 86.8 & 74.8 & 75.4 & 102.5 & 117.9 & 100.0 & 74.8 & 123.7 & 173.4 & 220.4 \\
\hline
\end{tabular}

Source: OECD, DSTI (STAN Industrial Database), 1996. 
DSTI/DOC(98)1

Annex Table 37: Trade-Weighted Relative Unit Labour Cost per Industry - Total price competitiveness

Italy

\begin{tabular}{|c|c|c|c|c|c|c|c|c|c|c|c|c|c|c|c|c|}
\hline & 1979 & 1980 & 1981 & 1982 & 1983 & 1984 & 1985 & 1986 & 1987 & 1988 & 1989 & 1990 & 1991 & 1992 & 1993 & 1994 \\
\hline Total manufacturing & 89.4 & 86.4 & 86.5 & 88.3 & 94.1 & 90.8 & 88.7 & 91.4 & 91.0 & 90.3 & 95.5 & 100.0 & 101.7 & 96.5 & 80.5 & 76.7 \\
\hline Food, drink and tobacco & 83.3 & 83.6 & 85.7 & 86.1 & 89.9 & 93.5 & 94.7 & 91.9 & 88.4 & 88.6 & 98.3 & 100.0 & 100.0 & 94.1 & 78.0 & 76.5 \\
\hline Textiles, Footwear and leather & 82.0 & 78.0 & 77.8 & 82.3 & 90.6 & 88.8 & 87.7 & 93.3 & 94.2 & 89.7 & 95.3 & 100.0 & 99.1 & 93.8 & 78.8 & 71.7 \\
\hline Wood, cork and furniture & 88.6 & 90.5 & 91.1 & 92.1 & 95.7 & 88.2 & 89.3 & 91.0 & 99.4 & 94.4 & 97.3 & 100.0 & 101.3 & 95.1 & 79.0 & 75.6 \\
\hline Paper and printing & 99.7 & 95.5 & 88.3 & 93.7 & 98.2 & 92.4 & 93.3 & 96.9 & 100.8 & 97.3 & 96.8 & 100.0 & 102.7 & 99.7 & 87.7 & 83.4 \\
\hline Chemicals & 121.5 & 108.0 & 97.9 & 91.7 & 99.2 & 92.2 & 88.9 & 92.3 & 90.7 & 92.0 & 96.1 & 100.0 & 101.9 & 99.4 & 83.0 & 78.9 \\
\hline Industrial chemicals & 127.1 & 111.8 & 103.6 & 96.5 & 104.8 & 99.1 & 98.8 & 102.1 & 102.9 & 110.0 & 106.5 & 100.0 & 103.1 & 102.1 & 84.3 & 81.1 \\
\hline Pharmaceuticals & 108.1 & 102.5 & 91.1 & 81.5 & 90.3 & 83.9 & 84.6 & 94.2 & 87.8 & 89.3 & 86.1 & 100.0 & 98.5 & 89.6 & 73.9 & 71.9 \\
\hline Petroleum products & 79.6 & 75.9 & 68.2 & 69.1 & 77.3 & 71.5 & 68.5 & 70.5 & 76.1 & 84.9 & 87.4 & 100.0 & 99.1 & 95.9 & 79.1 & 68.2 \\
\hline Rubber and plastic products & 93.8 & 88.3 & 82.4 & 83.1 & 93.0 & 90.7 & 80.4 & 79.2 & 80.2 & 74.8 & 92.1 & 100.0 & 102.2 & 101.5 & 82.8 & 78.8 \\
\hline Stone, clay and glass & 66.3 & 73.4 & 77.5 & 89.1 & 91.6 & 91.1 & 87.7 & 94.3 & 90.4 & 90.7 & 98.7 & 100.0 & 102.2 & 97.8 & 84.4 & 81.6 \\
\hline Basic metals & 95.6 & 76.3 & 79.9 & 79.0 & 91.1 & 85.6 & 82.3 & 93.1 & 92.1 & 91.0 & 99.3 & 100.0 & 105.2 & 102.7 & 80.9 & 75.6 \\
\hline Ferrous metals & 94.0 & 77.8 & 81.5 & 78.1 & 88.3 & 83.5 & 81.7 & 93.1 & 91.5 & 92.6 & 101.1 & 100.0 & 106.5 & 103.8 & 82.9 & 77.3 \\
\hline Non-ferrous metals & 104.2 & 75.0 & 78.4 & 78.5 & 90.3 & 89.2 & 81.3 & 85.3 & 91.8 & 87.2 & 95.8 & 100.0 & 100.6 & 99.3 & 76.4 & 72.8 \\
\hline Fabricated metal products and machinery & 86.5 & 85.9 & 86.2 & 87.3 & 91.3 & 86.8 & 85.1 & 87.1 & 87.2 & 87.2 & 92.5 & 100.0 & 103.5 & 98.0 & 81.3 & 77.4 \\
\hline Fabricated metal products & 100.7 & 100.5 & 101.9 & 96.0 & 99.9 & 98.8 & 98.3 & 97.9 & 92.3 & 91.5 & 95.8 & 100.0 & 100.0 & 91.5 & 79.8 & 73.3 \\
\hline Non-electrical machinery & 73.3 & 73.8 & 71.5 & 69.5 & 76.6 & 75.0 & 74.3 & 82.0 & 83.2 & 83.3 & 87.7 & 100.0 & 100.4 & 91.5 & 74.5 & 70.2 \\
\hline Electrical machinery & 87.6 & 90.9 & 90.0 & 92.8 & 97.0 & 96.0 & 98.3 & 91.5 & 87.7 & 87.2 & 94.3 & 100.0 & 106.1 & 104.1 & 88.7 & 90.1 \\
\hline Shipbuilding & 126.4 & 120.4 & 104.5 & 132.6 & 163.1 & 203.3 & 209.0 & 79.3 & 101.4 & 93.3 & 110.7 & 100.0 & 96.3 & 93.5 & 62.7 & 55.2 \\
\hline Other transport & 58.8 & 54.3 & 56.2 & 61.6 & 68.8 & 69.6 & 73.9 & 71.7 & 84.3 & 88.5 & 94.2 & 100.0 & 113.0 & 110.5 & 79.6 & 67.7 \\
\hline Motor vehicles & 116.4 & 108.0 & 104.7 & 104.4 & 109.8 & 101.3 & 96.7 & 93.0 & 93.6 & 89.3 & 89.6 & 100.0 & 113.1 & 108.3 & 86.0 & 83.6 \\
\hline Aerospace & 58.1 & 58.4 & 49.2 & 49.6 & 51.9 & 49.7 & 56.4 & 71.0 & 82.3 & 90.2 & 85.9 & 100.0 & 108.0 & 117.1 & 101.7 & 77.2 \\
\hline Instruments & 124.2 & 122.0 & 104.4 & 92.9 & 90.1 & 81.8 & 77.9 & 81.8 & 84.2 & 85.9 & 89.1 & 100.0 & 100.9 & 92.3 & 69.9 & 59.2 \\
\hline Other manufacturing industries & 61.5 & 65.1 & 78.9 & 76.1 & 85.2 & 72.3 & 69.5 & 86.1 & 96.0 & 93.3 & 89.3 & 100.0 & 98.5 & 89.6 & 74.4 & 67.6 \\
\hline
\end{tabular}

Annex Table 38: Trade-Weighted Relative Unit Labour Cost per Industry - Competitiveness among exporters

Italy

\begin{tabular}{|c|c|c|c|c|c|c|c|c|c|c|c|c|c|c|c|c|}
\hline & 1979 & 1980 & 1981 & 1982 & 1983 & 1984 & 1985 & 1986 & 1987 & 1988 & 1989 & 1990 & 1991 & 1992 & 1993 & 1994 \\
\hline Total manufacturing & 89.1 & 86.8 & 83.8 & 84.7 & 88.7 & 84.6 & 83.4 & 87.4 & 89.6 & 87.5 & 91.8 & 100.0 & 100.4 & 96.5 & 78.5 & 74.0 \\
\hline Food, drink and tobacco & 82.9 & 84.0 & 82.9 & 83.0 & 84.6 & 85.5 & 87.3 & 90.3 & 89.2 & 88.7 & 96.4 & 100.0 & 99.0 & 95.3 & 78.5 & 77.4 \\
\hline Textiles, Footwear and leather & 80.7 & 77.9 & 77.0 & 81.0 & 89.7 & 89.3 & 88.9 & 93.5 & 93.5 & 89.3 & 95.0 & 100.0 & 98.6 & 92.4 & 77.4 & 70.8 \\
\hline Wood, cork and furniture & 88.1 & 90.3 & 88.0 & 90.3 & 93.0 & 87.6 & 88.2 & 96.4 & 106.0 & 96.9 & 96.9 & 100.0 & 97.9 & 94.9 & 81.9 & 78.8 \\
\hline Paper and printing & 100.2 & 95.5 & 87.8 & 91.7 & 94.8 & 88.0 & 88.5 & 96.5 & 101.1 & 96.4 & 95.7 & 100.0 & 102.5 & 101.1 & 88.7 & 84.2 \\
\hline Chemicals & 119.2 & 105.3 & 93.4 & 86.3 & 92.9 & 85.3 & 84.1 & 88.1 & 90.2 & 90.0 & 93.4 & 100.0 & 101.4 & 100.1 & 82.0 & 77.1 \\
\hline Industrial chemicals & 125.8 & 109.9 & 99.2 & 91.4 & 99.4 & 91.3 & 92.6 & 96.9 & 102.6 & 108.6 & 104.1 & 100.0 & 103.3 & 103.7 & 84.3 & 80.5 \\
\hline Pharmaceuticals & 106.3 & 99.3 & 89.1 & 81.6 & 88.6 & 82.1 & 82.3 & 92.8 & 88.2 & 89.5 & 86.1 & 100.0 & 98.0 & 89.1 & 74.0 & 69.7 \\
\hline Petroleum products & 75.1 & 73.2 & 59.1 & 59.4 & 68.5 & 67.5 & 66.9 & 73.3 & 78.4 & 86.9 & 86.7 & 100.0 & 96.8 & 93.3 & 78.0 & 67.5 \\
\hline Rubber and plastic products & 94.7 & 89.3 & 83.4 & 83.3 & 91.9 & 87.8 & 77.9 & 77.2 & 80.0 & 72.7 & 88.6 & 100.0 & 101.6 & 102.5 & 82.5 & 77.5 \\
\hline Stone, clay and glass & 66.7 & 73.3 & 75.3 & 85.4 & 89.2 & 86.6 & 84.7 & 93.0 & 90.8 & 89.3 & 96.3 & 100.0 & 100.0 & 97.2 & 82.5 & 79.5 \\
\hline Basic metals & 99.2 & 81.1 & 82.3 & 82.4 & 90.5 & 86.5 & 83.3 & 89.9 & 91.9 & 89.0 & 95.2 & 100.0 & 102.9 & 99.3 & 75.2 & 71.1 \\
\hline Ferrous metals & 98.2 & 84.1 & 85.7 & 85.0 & 92.2 & 89.1 & 86.9 & 91.3 & 92.0 & 89.9 & 96.6 & 100.0 & 103.3 & 98.1 & 74.1 & 69.7 \\
\hline Non-ferrous metals & 102.8 & 78.0 & 74.4 & 73.7 & 83.2 & 83.6 & 76.8 & 81.8 & 91.3 & 87.4 & 92.5 & 100.0 & 101.7 & 100.4 & 77.1 & 73.9 \\
\hline Fabricated metal products and machinery & 82.8 & 84.7 & 81.0 & 81.3 & 83.5 & 78.7 & 78.4 & 81.3 & 83.9 & 83.3 & 87.9 & 100.0 & 102.1 & 97.9 & 79.5 & 74.7 \\
\hline Fabricated metal products & 97.6 & 97.8 & 96.7 & 92.6 & 95.1 & 92.3 & 93.2 & 95.0 & 90.8 & 89.2 & 92.8 & 100.0 & 98.9 & 91.8 & 79.9 & 73.7 \\
\hline Non-electrical machinery & 68.5 & 70.5 & 66.8 & 65.3 & 71.6 & 69.8 & 71.0 & 80.4 & 81.7 & 81.5 & 85.0 & 100.0 & 99.8 & 92.3 & 72.9 & 67.5 \\
\hline Electrical machinery & 72.1 & 77.8 & 74.9 & 78.7 & 83.7 & 82.3 & 86.1 & 83.5 & 82.1 & 82.7 & 89.5 & 100.0 & 104.5 & 102.8 & 88.1 & 87.4 \\
\hline Shipbuilding & 95.1 & 112.1 & 97.2 & 108.0 & 129.1 & 160.6 & 203.5 & 71.0 & 81.4 & 84.8 & 105.0 & 100.0 & 95.4 & 94.9 & 62.8 & 53.5 \\
\hline Other transport & 42.5 & 47.5 & 48.8 & 54.9 & 61.6 & 64.6 & 66.2 & 61.2 & 77.3 & 78.4 & 87.0 & 100.0 & 110.3 & 105.0 & 79.4 & 64.3 \\
\hline Motor vehicles & 119.1 & 117.2 & 106.9 & 106.1 & 107.1 & 96.0 & 89.9 & 86.5 & 91.7 & 85.8 & 84.9 & 100.0 & 107.6 & 105.6 & 84.5 & 79.8 \\
\hline Aerospace & 60.3 & 59.3 & 52.4 & 57.8 & 59.1 & 56.3 & 63.6 & 74.3 & 82.1 & 89.1 & 85.3 & 100.0 & 109.4 & 116.2 & 102.3 & 80.3 \\
\hline Instruments & 117.8 & 118.4 & 101.6 & 90.6 & 90.4 & 84.4 & 79.4 & 81.5 & 83.7 & 83.2 & 89.3 & 100.0 & 99.8 & 91.1 & 66.5 & 57.2 \\
\hline ther manufa & 56.6 & 67.6 & 83.9 & 87.8 & 105.8 & 90.7 & 89.6 & 97.6 & 100.6 & 93.7 & 88.0 & 100.0 & 101.1 & 92.8 & 76.3 & 14.9 \\
\hline
\end{tabular}

Source: OECD, DSTI (STAN Industrial Database), 1996. 
Annex Table 39: Trade-Weighted Relative Unit Labour Cost per Industry - Total price competitiveness

\begin{tabular}{|c|c|c|c|c|c|c|c|c|c|c|c|c|c|c|c|c|}
\hline & 1979 & 1980 & 1981 & 1982 & 1983 & 1984 & 1985 & 1986 & 1987 & 1988 & 1989 & 1990 & 1991 & 1992 & 1993 & 1994 \\
\hline Total manufacturing & 82.7 & 74.0 & 78.9 & 68.9 & 76.2 & 78.7 & 77.2 & 106.0 & 114.2 & 122.4 & 113.4 & 100.0 & 105.6 & 112.6 & 140.2 & 157.6 \\
\hline Food, drink and tobacco & 50.0 & 48.8 & 52.1 & 48.2 & 50.7 & 54.8 & 61.1 & 85.0 & 96.6 & 109.6 & 105.7 & 100.0 & 108.3 & 111.6 & 135.4 & 159.8 \\
\hline Textiles, Footwear and leather & 73.8 & 67.8 & 69.4 & 64.3 & 71.2 & 72.8 & 81.0 & 110.0 & 110.9 & 119.0 & 115.5 & 100.0 & 109.3 & 121.8 & 163.4 & 200.3 \\
\hline Wood, cork and furniture & 84.1 & 79.6 & 78.6 & 65.5 & 72.4 & 76.0 & 78.7 & 110.0 & 131.9 & 126.3 & 121.4 & 100.0 & 108.9 & 126.9 & 155.8 & 177.5 \\
\hline Paper and printing & 104.8 & 94.6 & 94.0 & 74.5 & 82.0 & 84.9 & 85.9 & 115.6 & 124.0 & 128.0 & 115.5 & 100.0 & 107.3 & 122.6 & 154.5 & 185.4 \\
\hline Chemicals & 63.7 & 71.4 & 68.7 & 58.2 & 71.1 & 72.5 & 72.0 & 106.8 & 112.8 & 126.0 & 114.1 & 100.0 & 106.6 & 104.3 & 130.0 & 145.7 \\
\hline Industrial chemicals & 65.6 & 72.4 & 69.5 & 57.5 & 71.6 & 71.3 & 72.2 & 105.1 & 112.6 & 125.5 & 114.2 & 100.0 & 102.8 & 103.3 & 128.3 & 147.4 \\
\hline Pharmaceuticals & 76.3 & 91.7 & 89.5 & 81.0 & 95.5 & 93.9 & 90.0 & 128.2 & 128.0 & 139.8 & 120.3 & 100.0 & 107.2 & 101.9 & 127.8 & 141.0 \\
\hline Petroleum products & 58.8 & 62.7 & 52.8 & 44.3 & 51.9 & 51.1 & 54.6 & 91.7 & 117.7 & 129.5 & 118.7 & 100.0 & 101.9 & 101.3 & 117.1 & 128.4 \\
\hline Rubber and plastic products & 56.8 & 66.6 & 67.6 & 60.0 & 74.3 & 84.5 & 78.3 & 110.8 & 112.8 & 125.4 & 116.3 & 100.0 & 109.5 & 105.4 & 134.2 & 155.8 \\
\hline Stone, clay and glass & 91.6 & 82.2 & 84.5 & 74.2 & 80.4 & 84.4 & 77.7 & 106.2 & 108.1 & 120.7 & 115.4 & 100.0 & 113.5 & 120.5 & 155.9 & 168.3 \\
\hline Basic metals & 58.7 & 54.0 & 70.2 & 60.2 & 75.7 & 71.4 & 75.9 & 120.1 & 113.9 & 113.6 & 109.4 & 100.0 & 113.6 & 123.7 & 164.9 & 165.7 \\
\hline Ferrous metals & 53.7 & 47.9 & 72.2 & 60.5 & 83.0 & 85.5 & 85.2 & 122.4 & 114.2 & 118.4 & 112.4 & 100.0 & 113.0 & 121.2 & 167.8 & 167.5 \\
\hline Non-ferrous metals & 58.9 & 57.8 & 61.6 & 58.9 & 68.7 & 73.2 & 89.1 & 139.3 & 128.9 & 127.6 & 112.7 & 100.0 & 118.7 & 135.8 & 197.8 & 208.8 \\
\hline Fabricated metal products and machinery & 115.4 & 87.4 & 91.6 & 80.5 & 86.8 & 89.8 & 81.3 & 106.5 & 118.1 & 125.7 & 115.2 & 100.0 & 101.5 & 109.3 & 134.4 & 151.1 \\
\hline Fabricated metal products & 97.2 & 90.5 & 90.9 & 75.2 & 81.0 & 88.4 & 84.0 & 104.4 & 115.4 & 125.6 & 122.4 & 100.0 & 106.1 & 110.3 & 133.1 & 150.5 \\
\hline Non-electrical machinery & 92.2 & 69.6 & 73.2 & 61.6 & 74.0 & 76.5 & 70.3 & 99.6 & 111.4 & 122.3 & 115.5 & 100.0 & 101.1 & 113.1 & 156.3 & 185.7 \\
\hline Electrical machinery & 168.9 & 126.8 & 126.8 & 100.7 & 103.5 & 95.1 & 91.2 & 113.1 & 120.6 & 121.6 & 112.1 & 100.0 & 100.9 & 112.7 & 134.9 & 160.9 \\
\hline Shipbuilding & 194.8 & 98.3 & 115.2 & 123.3 & 154.3 & 130.6 & 110.0 & 144.9 & 203.4 & 155.0 & 133.8 & 100.0 & 105.7 & 96.3 & 108.2 & 103.8 \\
\hline Other transport & & .. & .. & .. & .. & & & & & & & & & & .. & \\
\hline Motor vehicles & 105.0 & 72.5 & 93.4 & 91.8 & 100.2 & 113.3 & 109.9 & 128.9 & 113.1 & 122.9 & 119.3 & 100.0 & 107.4 & 107.6 & 121.4 & 146.0 \\
\hline Aerospace & 213.7 & 151.0 & 101.7 & 106.6 & 93.2 & 77.2 & 77.7 & 105.6 & 133.6 & 147.6 & 122.4 & 100.0 & 94.1 & 102.9 & 121.3 & 124.1 \\
\hline Instruments & 122.8 & 98.6 & 95.5 & 76.5 & 73.5 & 74.3 & 65.2 & 91.3 & 115.9 & 127.2 & 103.0 & 100.0 & 100.9 & 111.6 & 134.8 & 151.8 \\
\hline Other manufacturing industries & 80.1 & 68.8 & 77.2 & 71.1 & 66.7 & 76.3 & 77.6 & 98.1 & 96.6 & 112.5 & 105.6 & 100.0 & 110.7 & 113.1 & 140.1 & 160.7 \\
\hline
\end{tabular}

Annex Table 40: Trade-Weighted Relative Unit Labour Cost per Industry - Competitiveness among exporters

\begin{tabular}{|c|c|c|c|c|c|c|c|c|c|c|c|c|c|c|c|c|}
\hline & 1979 & 1980 & 1981 & 1982 & 1983 & 1984 & 1985 & 1986 & 1987 & 1988 & 1989 & 1990 & 1991 & 1992 & 1993 & 1994 \\
\hline Total manufacturing & 84.8 & 75.7 & 87.0 & 79.4 & 90.1 & 96.2 & 94.9 & 119.4 & 117.6 & 124.7 & 118.4 & 100.0 & 105.8 & 111.6 & 143.5 & $\overline{162.0}$ \\
\hline Food, drink and tobacco & 49.0 & 48.1 & 56.0 & 53.1 & 59.0 & 66.3 & 72.3 & 92.2 & 96.9 & 108.6 & 110.3 & 100.0 & 109.9 & 111.7 & 140.9 & 164.1 \\
\hline Textiles, Footwear and leather & 73.8 & 68.5 & 76.7 & 72.9 & 82.2 & 88.3 & 94.1 & 115.3 & 112.5 & 120.6 & 118.7 & 100.0 & 108.3 & 118.2 & 160.5 & 193.9 \\
\hline Wood, cork and furniture & 87.6 & 79.4 & 86.3 & 76.0 & 84.2 & 90.7 & 92.1 & 116.7 & 128.0 & 130.0 & 128.4 & 100.0 & 109.5 & 124.9 & 160.4 & 182.2 \\
\hline Paper and printing & 95.6 & 87.0 & 94.5 & 79.3 & 90.9 & 98.0 & 99.8 & 123.4 & 125.7 & 130.0 & 120.5 & 100.0 & 107.6 & 121.4 & 161.2 & 192.7 \\
\hline Chemicals & 64.4 & 74.1 & 75.4 & 67.9 & 84.4 & 90.4 & 89.5 & 120.0 & 114.0 & 127.5 & 118.3 & 100.0 & 106.7 & 104.2 & 133.5 & 150.6 \\
\hline Industrial chemicals & 67.4 & 76.6 & 77.7 & 68.5 & 85.5 & 89.1 & 89.4 & 118.7 & 113.7 & 126.3 & 118.1 & 100.0 & 103.7 & 102.7 & 132.8 & 153.4 \\
\hline Pharmaceuticals & 70.6 & 88.0 & 91.1 & 84.2 & 106.7 & 110.6 & 107.5 & 138.7 & 126.5 & 139.1 & 125.9 & 100.0 & 105.4 & 99.7 & 127.0 & 141.1 \\
\hline Petroleum products & 60.8 & 64.5 & 59.2 & 52.8 & 64.6 & 67.9 & 68.2 & 106.8 & 110.6 & 124.6 & 119.2 & 100.0 & 103.0 & 101.1 & 126.2 & 137.1 \\
\hline Rubber and plastic products & 57.0 & 65.7 & 69.6 & 65.0 & 80.6 & 93.4 & 92.1 & 118.0 & 114.5 & 129.2 & 120.7 & 100.0 & 109.1 & 106.9 & 139.2 & 161.3 \\
\hline Stone, clay and glass & 92.6 & 84.7 & 92.4 & 85.2 & 92.9 & 101.6 & 92.3 & 114.4 & 111.8 & 123.2 & 118.7 & 100.0 & 113.5 & 118.4 & 155.7 & 169.5 \\
\hline Basic metals & 56.9 & 49.8 & 72.3 & 66.8 & 89.5 & 86.8 & 94.3 & 128.0 & 116.5 & 120.3 & 117.9 & 100.0 & 111.9 & 120.0 & 160.6 & 167.2 \\
\hline Ferrous metals & 56.1 & 49.4 & 77.4 & 70.0 & 95.9 & 91.4 & 94.6 & 124.3 & 112.6 & 118.8 & 119.8 & 100.0 & 111.0 & 115.3 & 151.0 & 157.1 \\
\hline Non-ferrous metals & 55.3 & 49.4 & 57.3 & 57.5 & 74.8 & 82.0 & 101.3 & 140.8 & 130.4 & 131.5 & 117.6 & 100.0 & 115.6 & 133.3 & 196.1 & 210.3 \\
\hline Fabricated metal products and machinery & 122.9 & 92.7 & 103.5 & 94.1 & 102.9 & 109.5 & 101.5 & 124.3 & 123.8 & 128.2 & 120.3 & 100.0 & 101.6 & 108.7 & 137.5 & 154.8 \\
\hline Fabricated metal products & 100.6 & 93.4 & 100.2 & 87.4 & 99.6 & 111.9 & 105.4 & 115.0 & 118.5 & 127.0 & 127.4 & 100.0 & 106.3 & 108.9 & 134.0 & 150.7 \\
\hline Non-electrical machinery & 104.2 & 77.0 & 84.4 & 74.4 & 89.3 & 95.0 & 86.7 & 113.4 & 117.7 & 125.7 & 120.8 & 100.0 & 101.8 & 111.4 & 158.2 & 187.5 \\
\hline Electrical machinery & 199.6 & 142.0 & 152.2 & 127.4 & 132.7 & 127.0 & 120.9 & 137.6 & 132.4 & 130.1 & 119.3 & 100.0 & 99.2 & 108.2 & 130.9 & 152.5 \\
\hline Shipbuilding & 184.0 & 97.2 & 114.0 & 118.3 & 165.1 & 128.7 & 130.1 & 156.0 & 197.9 & 151.2 & 142.4 & 100.0 & 110.0 & 102.4 & 123.3 & 125.7 \\
\hline Other transport & 165.7 & 115.2 & 110.3 & 111.1 & 117.7 & 106.9 & 104.5 & 129.3 & 121.5 & 123.0 & 119.2 & 100.0 & 103.7 & 114.5 & 105.2 & 116.5 \\
\hline Motor vehicles & 96.2 & 70.0 & 87.9 & 89.2 & 93.6 & 104.2 & 101.5 & 124.6 & 110.1 & 121.9 & 118.1 & 100.0 & 105.7 & 107.6 & 124.9 & 147.3 \\
\hline Aerospace & 198.3 & 134.8 & 114.9 & 110.9 & 105.1 & 94.8 & 94.9 & 122.6 & 133.5 & 141.9 & 130.6 & 100.0 & 94.2 & 105.3 & 129.0 & 141.0 \\
\hline Instruments & 123.9 & 99.5 & 107.6 & 90.2 & 92.3 & 98.4 & 86.5 & 111.8 & 126.3 & 129.7 & 112.1 & 100.0 & 101.6 & 111.8 & 141.3 & 163.0 \\
\hline Other manufacturing industries & 89.2 & 81.7 & 96.8 & 97.4 & 96.7 & 110.3 & 109.8 & 118.6 & 104.8 & 116.7 & 108.8 & 100.0 & 114.4 & 118.8 & 149.6 & 179.7 \\
\hline
\end{tabular}

Source: OECD, DSTI (STAN Industrial Database), 1996 
DSTI/DOC(98)1

Annex Table 41: Trade-Weighted Relative Unit Labour Cost per Industry - Total price competitiveness

Netherlands

\begin{tabular}{|c|c|c|c|c|c|c|c|c|c|c|c|c|c|c|c|c|}
\hline & 1979 & 1980 & 1981 & 1982 & 1983 & 1984 & 1985 & 1986 & 1987 & 1988 & 1989 & 1990 & 1991 & 1992 & 1993 & 1994 \\
\hline Total manufacturing & 124.7 & 116.2 & 105.2 & 108.8 & 105.3 & 96.1 & 94.3 & 102.1 & 110.4 & 106.5 & 99.5 & 100.0 & 98.7 & 100.8 & 101.7 & 98.8 \\
\hline Food, drink and tobacco & 135.5 & 121.8 & 107.1 & 109.6 & 105.6 & 105.5 & 103.8 & 109.2 & 131.0 & 125.7 & 103.4 & 100.0 & 100.8 & 95.3 & 98.2 & 96.2 \\
\hline Textiles, Footwear and leather & .. & & .. & .. & & .. & .. & .. & & & & .. & .. & .. & .. & .. \\
\hline Wood, cork and furniture & 125.1 & 118.7 & 111.4 & 118.1 & 110.0 & 100.1 & 98.6 & 101.0 & 103.3 & 99.5 & 97.4 & 100.0 & 103.0 & 107.5 & 112.7 & 112.0 \\
\hline Paper and printing & 112.5 & 104.6 & 97.8 & 103.6 & 108.4 & 100.1 & 94.3 & 102.8 & 108.3 & 104.8 & 99.6 & 100.0 & 98.5 & 102.4 & 105.0 & 102.5 \\
\hline Chemicals & 128.9 & 123.6 & 114.8 & 118.9 & 109.9 & 100.4 & 98.8 & 104.8 & 109.2 & 104.2 & 99.2 & 100.0 & 97.1 & 100.2 & 102.3 & 97.2 \\
\hline Industrial chemicals & 136.3 & 123.9 & 99.6 & 100.3 & 98.2 & 86.5 & 83.9 & 97.6 & 105.6 & 101.2 & 94.7 & 100.0 & 95.6 & 99.3 & .. &.. \\
\hline Pharmaceuticals & 119.2 & 109.8 & 88.8 & 95.8 & 99.3 & 94.8 & 93.4 & 108.5 & 113.3 & 109.2 & 101.1 & 100.0 & 101.0 & 107.2 & 119.2 & 121.5 \\
\hline Petroleum products & 136.9 & 127.5 & 154.0 & 161.5 & 126.4 & 127.4 & & & 114.4 & 106.4 & 106.9 & 100.0 & 100.0 & 102.4 & 101.2 & 76.3 \\
\hline Rubber and plastic products & 112.8 & 108.9 & 89.0 & 98.0 & 95.9 & 89.2 & 86.6 & 96.3 & 101.2 & 99.8 & 97.9 & 100.0 & 98.3 & 102.2 & 100.5 & 101.1 \\
\hline Stone, clay and glass & 100.3 & 101.3 & 99.6 & 108.8 & 106.4 & 94.9 & 90.1 & 96.5 & 100.2 & 99.5 & 98.3 & 100.0 & 101.3 & 110.9 & 115.0 & 109.0 \\
\hline Basic metals & .. & .. & .. & .. & .. & & & .. & .. & .. & .. & .. & .. & .. & .. & .. \\
\hline Ferrous metals & .. & .. & .. & .. & .. & .. & .. & .. & .. & .. & .. & .. & .. & .. & .. & .. \\
\hline Non-ferrous metals & .. & .. & .. & .. & .. & .. & .. & .. & .. & .. & .. & .. & .. & .. & .. & .. \\
\hline Fabricated metal products and machinery & 121.8 & 113.4 & 100.8 & 103.8 & 101.0 & 88.6 & 87.5 & 98.9 & 106.3 & 104.5 & 99.6 & 100.0 & 97.2 & 100.1 & .. & 99.1 \\
\hline Fabricated metal products & 115.1 & 110.4 & 104.9 & 112.6 & 113.1 & 101.8 & 99.6 & 104.8 & 105.8 & 105.0 & 102.0 & 100.0 & 99.9 & 103.0 & 116.9 & 111.8 \\
\hline Non-electrical machinery & 98.2 & 95.9 & 81.2 & 85.1 & 87.4 & 75.8 & 77.9 & 93.0 & 99.8 & 98.4 & 96.7 & 100.0 & 95.7 & 99.6 & 102.8 & 97.9 \\
\hline Electrical machinery & & & & & & & & & & & & . & . & .. & .. & \\
\hline Shipbuilding & 220.7 & 213.9 & 220.6 & 194.7 & 157.5 & 145.9 & 134.1 & 139.0 & 101.7 & 99.5 & 92.6 & 100.0 & 90.8 & 91.1 & 90.9 & 78.5 \\
\hline Other transport & 108.7 & 83.9 & 80.2 & 67.4 & 68.4 & 67.8 & 75.1 & 85.4 & 90.4 & 103.4 & 96.8 & 100.0 & 95.3 & 96.0 & 86.0 & 77.8 \\
\hline Motor vehicles & .. & & .. & .. & & .. & .. & .. & & & & .. & .. & .. & .. & .. \\
\hline Aerospace & .. & .. & .. & .. & .. & .. & .. & .. & .. & .. & .. & .. & .. & .. & .. & .. \\
\hline Instruments & .. & .. & .. & .. & .. & .. & .. & .. & .. & .. & .. & .. & .. & .. & .. & .. \\
\hline
\end{tabular}

Annex Table 42: Trade-Weighted Relative Unit Labour Cost per Industry - Competitiveness among exporters

Netherlands

\begin{tabular}{|c|c|c|c|c|c|c|c|c|c|c|c|c|c|c|c|c|}
\hline & 1979 & 1980 & 1981 & 1982 & 1983 & 1984 & 1985 & 1986 & 1987 & 1988 & 1989 & 1990 & 1991 & 1992 & 1993 & 1994 \\
\hline Total manufacturing & 127.1 & 118.5 & 103.7 & 106.1 & 101.0 & 90.8 & 89.5 & 98.5 & 109.3 & 104.5 & 96.8 & 100.0 & 97.8 & 101.0 & 100.3 & 96.8 \\
\hline Food, drink and tobacco & 138.6 & 123.4 & 105.1 & 107.9 & 102.4 & 100.4 & 100.0 & 107.4 & 129.8 & 124.5 & 101.5 & 100.0 & 101.8 & 98.2 & 100.7 & 100.1 \\
\hline Textiles, Footwear and leather & 131.0 & 124.9 & 110.2 & 109.0 & 103.3 & 88.8 & 88.0 & 99.4 & 108.0 & 103.4 & 97.9 & 100.0 & 100.3 & 109.9 & 117.3 & 114.2 \\
\hline Wood, cork and furniture & 133.9 & 124.4 & 109.3 & 110.7 & 103.2 & 93.5 & 94.0 & 101.1 & 105.2 & 100.3 & 95.0 & 100.0 & 102.6 & 110.4 & 120.8 & 119.5 \\
\hline Paper and printing & 126.6 & 117.8 & 103.3 & 103.8 & 106.7 & 96.4 & 91.7 & 104.0 & 111.1 & 105.8 & 97.6 & 100.0 & 98.3 & 104.3 & 106.9 & 104.7 \\
\hline Chemicals & 124.4 & 118.2 & 108.4 & 110.9 & 102.0 & 91.8 & 91.3 & 98.9 & 108.3 & 102.7 & 97.4 & 100.0 & 96.9 & 101.2 & 102.1 & 95.8 \\
\hline Industrial chemicals & 131.5 & 119.6 & 96.0 & 96.2 & 93.8 & 81.7 & 80.0 & 92.9 & 104.9 & 100.6 & 93.6 & 100.0 & 95.9 & 100.3 & 92.2 & 90.2 \\
\hline Pharmaceuticals & 124.3 & 114.5 & 90.6 & 94.6 & 96.8 & 91.0 & 89.7 & 105.2 & 113.8 & 109.3 & 100.2 & 100.0 & 102.3 & 108.9 & 119.6 & 118.1 \\
\hline Petroleum products & 125.7 & 121.5 & 145.2 & 146.2 & 114.9 & 116.5 & 119.1 & 112.2 & 119.8 & 105.9 & 104.5 & 100.0 & 99.0 & 100.8 & 104.6 & 79.6 \\
\hline Rubber and plastic products & 122.2 & 115.1 & 91.3 & 97.4 & 93.4 & 85.3 & 84.0 & 94.9 & 103.1 & 100.0 & 96.0 & 100.0 & 97.6 & 102.9 & 102.3 & 103.3 \\
\hline Stone, clay and glass & 122.6 & 118.4 & 107.2 & 110.0 & 107.6 & 93.7 & 91.0 & 96.8 & 102.2 & 99.5 & 96.2 & 100.0 & 100.7 & 110.9 & 115.7 & 109.4 \\
\hline Basic metals & 83.5 & 80.3 & 70.2 & 74.4 & 82.5 & 75.2 & 78.4 & 88.4 & 103.2 & 98.4 & 92.4 & 100.0 & 95.4 & 102.6 & 95.7 & 92.1 \\
\hline Ferrous metals & 83.5 & 84.0 & 73.6 & 76.2 & 83.7 & 78.4 & 81.9 & 88.0 & 103.7 & 100.1 & 94.1 & 100.0 & 95.9 & 101.5 & 94.6 & 92.3 \\
\hline Non-ferrous metals & 87.5 & 78.7 & 64.4 & 73.3 & 81.1 & 70.0 & 71.8 & 88.8 & 101.4 & 94.2 & 88.8 & 100.0 & 94.8 & 104.7 & 97.6 & 90.8 \\
\hline Fabricated metal products & 119.4 & 113.3 & 97.1 & 99.0 & 95.4 & 83.3 & 82.9 & 94.3 & 103.6 & 101.6 & 96.1 & 100.0 & 96.2 & 99.9 & 97.9 & 96.0 \\
\hline Fabricated metal products & 120.0 & 115.1 & 103.4 & 108.4 & 107.1 & 95.2 & 94.8 & 104.2 & 108.4 & 105.9 & 99.4 & 100.0 & 99.2 & 105.0 & 117.3 & 113.6 \\
\hline Non-electrical machinery & 90.9 & 91.9 & 77.0 & 80.0 & 82.9 & 73.1 & 76.2 & 91.3 & 98.9 & 97.5 & 94.9 & 100.0 & 95.4 & 100.6 & 102.0 & 97.3 \\
\hline Electrical machinery & 155.8 & 163.4 & 126.8 & 132.7 & 96.0 & 81.9 & 83.6 & 85.5 & 101.5 & 91.5 & 98.4 & 100.0 & 115.3 & 130.5 & 125.0 & 127.8 \\
\hline Shipbuilding & 202.3 & 222.0 & 208.7 & 197.0 & 154.9 & 132.1 & 122.8 & 134.9 & 100.4 & 97.0 & 91.1 & 100.0 & 92.6 & 95.9 & 88.6 & 79.3 \\
\hline Other transport & 84.1 & 79.9 & 69.9 & 60.7 & 62.3 & 62.9 & 67.9 & 72.0 & 84.3 & 93.4 & 87.4 & 100.0 & 91.6 & 89.6 & 93.4 & 90.8 \\
\hline Motor vehicles & 88.8 & 80.0 & 77.9 & 71.6 & 74.0 & 72.7 & 80.7 & 84.8 & 97.4 & 100.4 & 90.3 & 100.0 & 96.1 & 99.3 & 105.2 & 101.8 \\
\hline Aerospace & 84.3 & 72.2 & 64.2 & 69.9 & 59.5 & 56.5 & 66.4 & 88.0 & 111.2 & 109.0 & 94.6 & 100.0 & 90.3 & 93.0 & 82.7 & 78.6 \\
\hline Instruments & 79.6 & 78.5 & 66.6 & 67.6 & 66.8 & 59.0 & 61.4 & 78.0 & 87.9 & 90.1 & 79.5 & 100.0 & 95.4 & 98.2 & 97.3 & 80.0 \\
\hline & & & 992 & & 970 & 885 & 921 & 911 & 929 & 98. & & & & & & \\
\hline
\end{tabular}

Source: OECD, DSTI (STAN Industrial Database), 1996. 
Annex Table 43: Trade-Weighted Relative Unit Labour Cost per Industry - Total price competitiveness

Norway

\begin{tabular}{|c|c|c|c|c|c|c|c|c|c|c|c|c|c|c|c|c|}
\hline & 1979 & 1980 & 1981 & 1982 & 1983 & 1984 & 1985 & 1986 & 1987 & 1988 & 1989 & 1990 & 1991 & 1992 & 1993 & 1994 \\
\hline Total manufacturing & 92.5 & 93.4 & 98.6 & 101.2 & 102.3 & 99.2 & 100.0 & 100.0 & 101.2 & 106.6 & 102.6 & 100.0 & 96.8 & 90.5 & 89.6 & 92.5 \\
\hline Food, drink and tobacco & 70.6 & 72.3 & 83.0 & 86.3 & 91.4 & 91.3 & 92.3 & 95.7 & 97.2 & 103.3 & 99.4 & 100.0 & 97.0 & 92.0 & 90.0 & 91.8 \\
\hline Textiles, Footwear and leather & 123.9 & 115.5 & 117.2 & 125.0 & 120.2 & 115.7 & 113.3 & 106.2 & 110.1 & 107.1 & 108.8 & 100.0 & 91.9 & 89.9 & 92.8 & 94.7 \\
\hline Wood, cork and furniture & 88.1 & 87.5 & 89.5 & 105.4 & 116.7 & 111.5 & 104.8 & 99.8 & 105.3 & 110.3 & 103.8 & 100.0 & 97.1 & 80.6 & 89.5 & 92.5 \\
\hline Paper and printing & 96.4 & 98.5 & 95.8 & 98.9 & 107.5 & 101.1 & 100.3 & 102.0 & 109.8 & 111.3 & 105.1 & 100.0 & 98.0 & 92.8 & 102.5 & 105.3 \\
\hline Chemicals & 140.6 & 137.8 & 140.1 & 133.8 & 136.1 & 120.8 & 122.9 & 132.2 & 107.7 & 113.9 & 112.6 & 100.0 & 107.6 & 106.0 & 107.6 & 111.3 \\
\hline Industrial chemicals & 137.9 & 132.8 & 129.3 & 122.2 & 120.0 & 101.8 & 112.4 & 130.4 & 106.2 & 107.1 & 110.3 & 100.0 & 101.9 & 103.2 & 104.4 & 107.6 \\
\hline Pharmaceuticals & 136.1 & 118.1 & 124.1 & 134.3 & 152.9 & 134.7 & 159.6 & 125.8 & 120.9 & 131.0 & 116.1 & 100.0 & 91.1 & 103.7 & 103.5 & 112.1 \\
\hline Petroleum products & 160.5 & 225.1 & 278.9 & 204.1 & 246.7 & 237.6 & 183.2 & 217.3 & 101.6 & 129.1 & 141.6 & 100.0 & 187.4 & 174.3 & 177.6 & 172.0 \\
\hline Rubber and plastic products & 114.6 & 109.2 & 114.9 & 110.6 & 110.3 & 111.3 & 101.0 & 99.7 & 98.5 & 107.7 & 101.3 & 100.0 & 99.1 & 93.7 & 99.5 & 102.0 \\
\hline Stone, clay and glass & 81.1 & 77.3 & 78.6 & 87.8 & 92.5 & 101.0 & 102.9 & 104.3 & 101.9 & 114.4 & 105.1 & 100.0 & 112.5 & 96.5 & 101.1 & 103.6 \\
\hline Basic metals & 94.5 & 105.1 & 113.6 & 112.2 & 90.6 & 95.7 & 99.4 & 105.6 & 100.9 & 100.2 & 103.3 & 100.0 & 94.5 & 92.2 & 90.0 & 92.5 \\
\hline Ferrous metals & 93.5 & 112.6 & 99.1 & 107.8 & 89.6 & 91.6 & 89.2 & 93.9 & 99.4 & 93.4 & 96.9 & 100.0 & 91.9 & 85.0 & 82.2 & 85.0 \\
\hline Non-ferrous metals & 98.8 & 99.9 & 125.7 & 120.8 & 91.9 & 97.1 & 103.3 & 114.0 & 101.8 & 101.0 & 104.6 & 100.0 & 94.9 & 95.4 & 93.5 & 90.8 \\
\hline Fabricated metal products and machinery & 88.7 & 90.0 & 91.9 & 94.1 & 96.1 & 92.8 & 91.9 & 90.6 & 96.0 & 104.5 & 99.8 & 100.0 & 94.5 & 87.5 & 84.4 & 88.4 \\
\hline Fabricated metal products & 82.5 & 82.8 & 90.0 & 94.3 & 99.7 & 99.1 & 97.0 & 93.3 & 101.9 & 110.9 & 107.7 & 100.0 & 92.7 & 82.5 & 88.0 & 92.0 \\
\hline Non-electrical machinery & 98.8 & 92.7 & 94.8 & 92.0 & 106.3 & 100.9 & 97.7 & 99.1 & 105.9 & 108.9 & 99.9 & 100.0 & 89.1 & 84.4 & 85.7 & 88.1 \\
\hline Electrical machinery & 63.3 & 66.4 & 63.0 & 69.2 & 70.1 & 76.8 & 89.0 & 87.3 & 86.8 & 96.0 & 94.5 & 100.0 & 100.9 & 97.1 & 97.6 & 109.4 \\
\hline Shipbuilding & 70.3 & 98.8 & 99.2 & 104.1 & 84.3 & 85.0 & .. & & 61.3 & 92.8 & 88.8 & 100.0 & 88.7 & 81.5 & 67.9 & 63.6 \\
\hline Other transport & 90.9 & 78.4 & 84.9 & 92.1 & 97.5 & 112.1 & 112.5 & 112.4 & 115.3 & 113.5 & 115.1 & 100.0 & 132.6 & 123.1 & 89.0 & 97.8 \\
\hline Motor vehicles & 102.0 & 102.0 & 118.2 & 122.9 & 114.6 & 93.4 & 88.0 & 85.3 & 97.9 & 108.6 & 92.7 & 100.0 & 98.4 & 95.9 & 77.1 & 78.9 \\
\hline Aerospace & 92.9 & 121.5 & 72.7 & 80.8 & 87.4 & 80.5 & 78.5 & 110.7 & 100.8 & 105.7 & 94.2 & 100.0 & 78.6 & 68.8 & 78.9 & 79.8 \\
\hline Instruments & & & & & & & .. & .. & .. & .. & .. & .. & .. & .. & .. & \\
\hline Other manufacturing industries & 66.1 & 74.1 & 75.9 & 82.6 & 70.9 & 73.7 & 90.7 & 93.0 & 93.1 & 107.6 & 102.4 & 100.0 & 71.1 & 69.7 & 70.9 & 72.7 \\
\hline
\end{tabular}

Annex Table 44: Trade-Weighted Relative Unit Labour Cost per Industry - Competitiveness among exporters

Norway

\begin{tabular}{|c|c|c|c|c|c|c|c|c|c|c|c|c|c|c|c|c|}
\hline & 1979 & 1980 & 1981 & 1982 & 1983 & 1984 & 1985 & 1986 & 1987 & 1988 & 1989 & 1990 & 1991 & 1992 & 1993 & $\overline{1994}$ \\
\hline Total manufacturing & 91.9 & 93.3 & 96.6 & 95.7 & 93.5 & 90.6 & 92.0 & 94.3 & 97.2 & 103.2 & 100.0 & 100.0 & 96.6 & 89.4 & 83.0 & 85.0 \\
\hline Food, drink and tobacco & 68.9 & 71.3 & 81.5 & 83.0 & 83.6 & 83.8 & 85.8 & 91.0 & 92.9 & 100.1 & 98.1 & 100.0 & 95.6 & 89.5 & 83.7 & 86.3 \\
\hline Textiles, Footwear and leather & 115.6 & 110.1 & 113.1 & 118.9 & 111.2 & 105.3 & 105.8 & 100.7 & 105.5 & 104.7 & 107.2 & 100.0 & 91.3 & 89.9 & 88.5 & 90.6 \\
\hline Wood, cork and furniture & 85.8 & 88.7 & 89.5 & 91.9 & 93.9 & 92.9 & 93.0 & 93.5 & 99.5 & 106.7 & 100.0 & 100.0 & 99.4 & 84.4 & 82.0 & 85.0 \\
\hline Paper and printing & 96.8 & 99.9 & 95.0 & 91.8 & 94.0 & 89.0 & 90.7 & 95.5 & 105.0 & 108.7 & 102.5 & 100.0 & 95.8 & 89.2 & 88.6 & 91.0 \\
\hline Chemicals & 139.3 & 133.4 & 137.3 & 124.1 & 123.2 & 109.9 & 115.0 & 126.0 & 105.4 & 112.6 & 112.0 & 100.0 & 106.6 & 104.1 & 99.1 & 102.2 \\
\hline Industrial chemicals & 131.4 & 125.0 & 123.9 & 113.6 & 108.9 & 92.8 & 104.8 & 123.8 & 105.2 & 105.1 & 109.7 & 100.0 & 101.1 & 102.4 & 98.2 & 102.4 \\
\hline Pharmaceuticals & 133.2 & 105.2 & 111.7 & 113.4 & 127.3 & 116.8 & 142.6 & 119.9 & 113.6 & 124.2 & 111.1 & 100.0 & 90.3 & 99.6 & 92.7 & 96.2 \\
\hline Petroleum products & 176.6 & 239.5 & 288.5 & 189.4 & 225.7 & 218.7 & 175.6 & 206.6 & 101.8 & 135.5 & 142.1 & 100.0 & 186.8 & 168.0 & 161.8 & 159.3 \\
\hline Rubber and plastic products & 109.9 & 100.1 & 108.8 & 101.3 & 99.3 & 101.5 & 93.7 & 94.3 & 96.0 & 106.3 & 101.6 & 100.0 & 97.9 & 89.1 & 84.5 & 87.6 \\
\hline Stone, clay and glass & 84.2 & 80.2 & 81.4 & 82.6 & 83.3 & 89.8 & 95.9 & 98.1 & 97.2 & 110.6 & 102.2 & 100.0 & 115.0 & 99.7 & 94.8 & 97.3 \\
\hline Basic metals & 97.5 & 115.4 & 120.3 & 112.0 & 87.9 & 93.9 & 98.9 & 102.1 & 98.0 & 100.0 & 104.8 & 100.0 & 94.2 & 89.8 & 85.1 & 87.1 \\
\hline Ferrous metals & 97.3 & 124.8 & 110.1 & 107.6 & 85.6 & 88.9 & 87.7 & 89.4 & 95.0 & 92.3 & 97.6 & 100.0 & 91.0 & 81.2 & 76.8 & 77.9 \\
\hline Non-ferrous metals & 99.1 & 108.7 & 121.4 & 115.2 & 88.4 & 94.3 & 102.9 & 113.9 & 101.7 & 103.6 & 107.2 & 100.0 & 95.4 & 95.7 & 90.4 & 91.2 \\
\hline Fabricated metal products and machinery & 87.2 & 89.1 & 88.1 & 88.1 & 87.7 & 83.8 & 83.3 & 84.7 & 91.8 & 100.1 & 96.3 & 100.0 & 94.8 & 86.8 & 78.8 & 81.5 \\
\hline Fabricated metal products & 83.6 & 82.9 & 89.6 & 90.3 & 91.6 & 90.2 & 89.5 & 88.3 & 98.7 & 107.2 & 105.7 & 100.0 & 92.8 & 81.8 & 79.6 & 82.0 \\
\hline Non-electrical machinery & 92.0 & 88.3 & 88.5 & 85.5 & 95.3 & 89.0 & 87.1 & 91.4 & 99.1 & 103.9 & 96.3 & 100.0 & 90.8 & 83.8 & 77.1 & 77.0 \\
\hline Electrical machinery & 58.9 & 62.4 & 56.2 & 62.1 & 62.5 & 67.4 & 77.2 & 75.9 & 78.9 & 90.6 & 92.0 & 100.0 & 98.8 & 91.2 & 85.2 & 91.9 \\
\hline Shipbuilding & 64.6 & 89.5 & 92.3 & 90.8 & 79.3 & 85.6 & 76.6 & 69.0 & 64.9 & 90.1 & 88.3 & 100.0 & 88.8 & 82.4 & 68.5 & 67.3 \\
\hline Other transport & 59.5 & 63.6 & 65.8 & 73.2 & 78.8 & 86.7 & 92.2 & 91.3 & 101.7 & 100.2 & 103.7 & 100.0 & 127.5 & 115.7 & 84.2 & 85.8 \\
\hline Motor vehicles & 99.6 & 100.1 & 113.1 & 114.3 & 100.0 & 81.9 & 78.7 & 78.5 & 90.0 & 101.9 & 89.6 & 100.0 & 99.1 & 95.9 & 73.7 & 73.1 \\
\hline Aerospace & 85.0 & 113.7 & 79.0 & 87.0 & 93.8 & 96.6 & 91.9 & 117.8 & 98.5 & 100.8 & 96.7 & 100.0 & 79.2 & 68.9 & 80.9 & 78.1 \\
\hline Instruments & 55.0 & 74.2 & 63.5 & 60.2 & 53.6 & 56.8 & 57.8 & 68.3 & 77.7 & 83.7 & 81.8 & 100.0 & 73.0 & 62.2 & 54.0 & 53.7 \\
\hline Other manufa & 63.8 & 88.7 & 85.0 & 82.7 & 74.6 & 75.7 & 90.9 & 90.4 & 90.0 & 102.3 & 95.6 & 100.0 & 73.0 & 69.1 & 64.2 & \\
\hline
\end{tabular}

Source: OECD, DSTI (STAN Industrial Database), 1996. 
DSTI/DOC(98)1

Annex Table 45: Trade-Weighted Relative Unit Labour Cost per Industry - Total price competitiveness

Portugal

\begin{tabular}{|c|c|c|c|c|c|c|c|c|c|c|c|c|c|c|c|c|}
\hline & 1979 & 1980 & 1981 & 1982 & 1983 & 1984 & 1985 & 1986 & 1987 & 1988 & 1989 & 1990 & 1991 & 1992 & 1993 & 1994 \\
\hline $\begin{array}{l}\text { Total manufacturing } \\
\end{array}$ & 98.0 & 97.7 & 106.5 & 100.9 & 89.0 & 89.2 & 88.9 & 90.7 & 91.3 & 91.9 & 92.9 & 100.0 & 108.3 & 120.0 & 108.9 & 108.4 \\
\hline Food, drink and tobacco & 139.5 & 144.8 & 136.2 & 125.8 & 102.9 & 98.1 & 103.1 & 100.1 & 93.9 & 86.7 & 87.8 & 100.0 & 110.2 & 121.9 & 114.2 & 109.9 \\
\hline Textiles, Footwear and leather & 100.0 & 95.6 & 109.1 & 100.2 & 93.4 & 85.5 & 82.0 & 88.5 & 94.8 & 89.7 & 90.8 & 100.0 & 106.7 & 119.1 & 114.6 & 115.1 \\
\hline Wood, cork and furniture & 80.9 & 81.8 & 91.3 & 90.2 & 72.1 & 82.0 & 77.0 & 94.4 & 95.6 & 102.2 & 99.0 & 100.0 & 108.2 & 115.1 & 107.9 & 137.8 \\
\hline Paper and printing & 86.4 & 79.3 & 88.4 & 87.9 & 83.1 & 85.0 & 74.0 & 79.3 & 86.9 & 80.0 & 93.3 & 100.0 & 99.8 & 102.8 & 99.8 & 88.6 \\
\hline Chemicals & 67.5 & 63.8 & 80.3 & 74.7 & 79.7 & 81.9 & 79.2 & 82.2 & 81.4 & 101.9 & 90.9 & 100.0 & 114.7 & 136.8 & 112.5 & 102.1 \\
\hline Industrial chemicals & 71.5 & 67.2 & 92.2 & 82.2 & 86.9 & 85.7 & 86.4 & 88.6 & 88.4 & 107.1 & 95.0 & 100.0 & 107.3 & 124.6 & 103.2 & 89.4 \\
\hline Pharmaceuticals & 31.8 & 31.9 & 39.0 & 35.1 & 39.3 & 41.6 & 44.6 & 51.8 & 53.0 & 72.9 & 73.8 & 100.0 & 137.6 & 159.5 & 139.9 & 180.2 \\
\hline Petroleum products & 57.7 & 48.4 & 53.8 & 47.6 & 54.8 & 69.6 & 58.0 & 60.0 & 81.5 & 99.9 & 86.8 & 100.0 & 100.4 & 98.6 & 72.2 & 63.4 \\
\hline Rubber and plastic products & 86.4 & 76.7 & 86.2 & 87.2 & 91.7 & 97.9 & 94.3 & 93.8 & 86.1 & 109.6 & 93.6 & 100.0 & 114.4 & 172.8 & 133.3 & 97.7 \\
\hline Stone, clay and glass & 101.9 & 96.5 & 106.2 & 103.6 & 96.6 & 89.9 & 86.1 & 87.5 & 85.8 & 93.8 & 95.6 & 100.0 & 107.8 & 119.6 & 110.2 & 113.1 \\
\hline Basic metals & 109.8 & 101.3 & 124.1 & 106.8 & 111.0 & 108.4 & 124.3 & 125.7 & 119.4 & 145.3 & 114.8 & 100.0 & 109.9 & 117.1 & 107.7 & 68.3 \\
\hline Ferrous metals & 107.0 & 101.9 & 123.3 & 105.5 & 108.5 & 105.7 & 121.6 & 124.7 & 117.4 & 143.9 & 115.5 & 100.0 & 112.4 & 119.6 & 109.0 & 58.9 \\
\hline Non-ferrous metals & 133.8 & 117.2 & 139.7 & 116.9 & 127.2 & 121.2 & 129.7 & 120.3 & 126.5 & 156.3 & 113.8 & 100.0 & 99.4 & 105.9 & 103.0 & 129.2 \\
\hline Fabricated metal products and machinery & 106.6 & 108.4 & 116.7 & 109.5 & 92.1 & 89.5 & 99.6 & 98.0 & 99.5 & 96.4 & 98.4 & 100.0 & 110.1 & 123.5 & 111.7 & 115.6 \\
\hline Fabricated metal products & 99.6 & 112.9 & 143.3 & 133.5 & 121.8 & 100.4 & 126.3 & 90.0 & 107.6 & 103.9 & 100.1 & 100.0 & 108.7 & 121.2 & 115.5 & 110.0 \\
\hline Non-electrical machinery & 99.6 & 133.2 & 139.5 & 99.4 & 85.2 & 89.7 & 108.0 & 110.5 & 110.2 & 109.0 & 100.4 & 100.0 & 110.1 & 131.0 & 126.3 & 100.7 \\
\hline Electrical machinery & 70.5 & 73.3 & 84.5 & 84.0 & 68.1 & 71.3 & 71.7 & 92.3 & 91.0 & 88.9 & 91.0 & 100.0 & 104.3 & 114.0 & 105.2 & 105.0 \\
\hline Shipbuilding & 107.5 & 122.0 & 108.5 & 112.4 & 94.1 & 73.6 & 72.0 & 91.0 & 84.7 & 85.6 & 89.8 & 100.0 & 102.8 & 124.5 & 95.4 & 82.7 \\
\hline Other transport & 88.3 & 88.1 & 97.5 & 93.9 & 89.7 & 81.5 & 88.6 & 92.2 & 101.8 & 97.9 & 95.4 & 100.0 & 104.9 & 111.6 & 93.2 & 98.0 \\
\hline Motor vehicles & 115.2 & 111.4 & 120.1 & 113.5 & 102.8 & 92.1 & 102.8 & 101.0 & 103.7 & 100.5 & 100.6 & 100.0 & 110.5 & 124.2 & 105.6 & 105.6 \\
\hline Aerospace & & .. & .. & & & & & & & & & .. & & .. & .. & \\
\hline Instruments & 63.6 & .. & & 79.1 & 68.7 & 57.2 & 58.7 & 80.6 & 103.6 & 86.4 & 87.9 & .. & 101.5 & 112.2 & 109.4 & 104.5 \\
\hline Other manufacturing industries & 155.6 & 131.1 & 143.2 & 134.4 & 106.3 & 109.6 & 109.6 & 103.4 & 108.8 & 105.5 & 102.6 & 100.0 & 99.0 & 103.0 & 99.7 & 97.4 \\
\hline
\end{tabular}

Annex Table 46: Trade-Weighted Relative Unit Labour Cost per Industry - Competitiveness among exporters

Portugal

\begin{tabular}{|c|c|c|c|c|c|c|c|c|c|c|c|c|c|c|c|c|}
\hline & 1979 & 1980 & 1981 & 1982 & 1983 & 1984 & 1985 & 1986 & 1987 & 1988 & 1989 & 1990 & 1991 & 1992 & 1993 & 1994 \\
\hline Total manufacturing & 97.3 & 98.9 & 104.7 & 97.4 & 86.6 & 83.3 & 82.7 & 85.7 & 87.9 & 88.0 & 89.1 & 100.0 & 107.5 & 119.4 & 104.0 & 101.9 \\
\hline Food, drink and tobacco & 132.9 & 142.0 & 132.8 & 124.6 & 99.5 & 94.8 & 97.1 & 97.0 & 88.3 & 83.6 & 85.6 & 100.0 & 109.6 & 119.4 & 107.5 & 101.9 \\
\hline Textiles, Footwear and leather & 97.1 & 97.5 & 111.3 & 102.0 & 93.7 & 87.1 & 82.6 & 87.1 & 93.2 & 88.6 & 89.6 & 100.0 & 106.6 & 118.6 & 113.1 & 113.0 \\
\hline Wood, cork and furniture & 76.1 & 81.3 & 88.9 & 85.6 & 71.1 & 77.8 & 75.9 & 91.4 & 92.4 & 100.2 & 96.8 & 100.0 & 108.8 & 117.9 & 111.6 & 143.4 \\
\hline Paper and printing & 86.7 & 80.2 & 87.8 & 85.8 & 79.0 & 79.1 & 66.9 & 75.0 & 84.6 & 77.8 & 89.7 & 100.0 & 100.8 & 108.3 & 100.9 & 89.4 \\
\hline Chemicals & 65.5 & 63.0 & 77.0 & 70.3 & 73.9 & 73.3 & 72.0 & 76.3 & 78.3 & 97.2 & 86.8 & 100.0 & 113.8 & 135.5 & 106.0 & 94.4 \\
\hline Industrial chemicals & 69.0 & 65.1 & 86.8 & 76.6 & 80.6 & 76.0 & 77.6 & 81.6 & 85.7 & 103.6 & 91.9 & 100.0 & 107.0 & 123.6 & 96.9 & 83.1 \\
\hline Pharmaceuticals & 31.8 & 31.5 & 38.6 & 34.9 & 38.8 & 41.4 & 43.8 & 50.1 & 52.9 & 72.1 & 72.7 & 100.0 & 137.5 & 159.6 & 139.6 & 172.4 \\
\hline Petroleum products & 59.8 & 50.8 & 49.0 & 47.7 & 50.9 & 61.5 & 54.0 & 59.4 & 80.5 & 100.6 & 84.0 & 100.0 & 96.2 & 94.9 & 67.8 & 60.6 \\
\hline Rubber and plastic products & 84.3 & 78.1 & 86.9 & 85.8 & 89.6 & 92.1 & 87.2 & 87.9 & 81.1 & 101.4 & 88.2 & 100.0 & 112.1 & 167.8 & 124.2 & 89.9 \\
\hline Stone, clay and glass & 102.7 & 97.5 & 103.1 & 97.3 & 90.4 & 85.6 & 83.1 & 84.3 & 82.1 & 89.3 & 93.1 & 100.0 & 106.2 & 118.9 & 105.0 & 105.8 \\
\hline Basic metals & 111.6 & 102.8 & 125.0 & 104.6 & 103.4 & 103.3 & 118.9 & 115.9 & 112.4 & 135.8 & 106.9 & 100.0 & 109.9 & 120.7 & 103.8 & 69.0 \\
\hline Ferrous metals & 106.9 & 102.2 & 125.4 & 104.2 & 102.3 & 105.1 & 122.8 & 115.6 & 110.9 & 134.9 & 108.1 & 100.0 & 112.0 & 121.5 & 103.7 & 58.7 \\
\hline Non-ferrous metals & 141.8 & 126.2 & 139.4 & 115.3 & 113.9 & 107.7 & 116.1 & 111.8 & 117.4 & 144.1 & 105.7 & 100.0 & 99.8 & 111.7 & 103.7 & 131.8 \\
\hline Fabricated metal products and machinery & 102.1 & 107.1 & 110.3 & 102.5 & 89.3 & 82.5 & 90.7 & 90.9 & 94.2 & 90.9 & 93.4 & 100.0 & 109.1 & 123.2 & 108.0 & 110.0 \\
\hline Fabricated metal products & 98.0 & 113.2 & 138.3 & 128.8 & 113.6 & 91.7 & 118.6 & 85.8 & 102.4 & 99.2 & 96.5 & 100.0 & 109.2 & 122.1 & 113.3 & 103.9 \\
\hline Non-electrical machinery & 92.6 & 126.3 & 124.1 & 86.3 & 76.8 & 80.7 & 100.5 & 103.2 & 104.2 & 103.1 & 95.0 & 100.0 & 109.6 & 130.7 & 118.0 & 90.8 \\
\hline Electrical machinery & 58.0 & 63.5 & 70.2 & 69.7 & 58.0 & 59.1 & 60.6 & 81.4 & 82.2 & 82.6 & 85.4 & 100.0 & 103.2 & 114.0 & 103.8 & 102.1 \\
\hline Shipbuilding & 80.3 & 106.6 & 104.0 & 103.5 & 81.2 & 77.1 & 82.0 & 80.4 & 74.2 & 79.4 & 86.4 & 100.0 & 107.2 & 121.5 & 97.0 & 99.1 \\
\hline Other transport & 66.8 & 75.5 & 81.6 & 83.0 & 79.6 & 77.4 & 89.8 & 86.3 & 93.0 & 88.2 & 89.7 & 100.0 & 105.7 & 111.3 & 88.9 & 91.0 \\
\hline Motor vehicles & 115.4 & 114.0 & 128.1 & 120.0 & 105.5 & 94.6 & 104.1 & 99.3 & 100.3 & 94.3 & 95.9 & 100.0 & 107.4 & 120.4 & 102.5 & 103.6 \\
\hline Aerospace & 122.9 & 115.1 & 105.8 & 104.7 & 91.3 & 89.2 & 89.0 & 93.1 & 103.9 & 99.4 & 97.5 & 100.0 & 107.5 & 121.8 & 105.9 & 109.8 \\
\hline Instruments & 59.1 & 77.5 & 82.1 & 76.6 & 69.0 & 59.7 & 61.2 & 81.4 & 103.6 & 86.3 & 89.7 & 100.0 & 100.6 & 110.8 & 104.5 & 105.1 \\
\hline & & & & & & & & & 1075 & & 968 & & & & & \\
\hline
\end{tabular}

Source: OECD, DSTI (STAN Industrial Database), 1996. 
Annex Table 47: Trade-Weighted Relative Unit Labour Cost per Industry - Total price competitiveness

\begin{tabular}{|c|c|c|c|c|c|c|c|c|c|c|c|c|c|c|c|c|}
\hline & 1979 & 1980 & 1981 & 1982 & 1983 & 1984 & 1985 & 1986 & 1987 & 1988 & 1989 & 1990 & 1991 & 1992 & 1993 & 1994 \\
\hline Total manufacturing & 103.3 & 101.9 & 93.9 & 90.7 & 80.2 & 79.8 & 76.2 & 78.3 & 78.7 & 84.2 & 91.9 & 100.0 & 99.7 & 98.0 & 90.8 & 88.7 \\
\hline Food, drink and tobacco & 99.8 & 100.9 & 90.4 & 86.6 & 72.7 & 72.3 & 67.8 & 77.0 & 77.6 & 82.3 & 90.3 & 100.0 & 99.0 & 95.3 & 85.2 & 84.7 \\
\hline Textiles, Footwear and leather & 96.8 & 106.7 & 100.4 & 88.5 & 77.6 & 75.4 & 73.1 & 77.5 & 79.1 & 85.9 & 90.5 & 100.0 & 100.4 & 97.1 & 100.5 & 92.1 \\
\hline Wood, cork and furniture & 97.2 & 108.4 & 94.2 & 81.4 & 75.9 & 76.9 & 78.3 & 73.3 & 76.6 & 85.4 & 94.2 & 100.0 & 101.6 & 99.3 & 96.9 & 96.9 \\
\hline Paper and printing & 102.1 & 86.5 & 83.1 & 82.3 & 71.6 & 66.1 & 64.9 & 72.5 & 74.0 & 83.4 & 91.3 & 100.0 & 103.8 & 112.2 & 106.8 & 108.4 \\
\hline Chemicals & 95.7 & 95.2 & 90.4 & 90.4 & 85.2 & 83.3 & 78.3 & 84.7 & 82.3 & 83.4 & 90.7 & 100.0 & 98.0 & 94.1 & 84.4 & 84.8 \\
\hline Industrial chemicals & 94.1 & 91.5 & 89.1 & 87.5 & 81.3 & 81.6 & 81.6 & 84.8 & 82.4 & 82.9 & 91.0 & 100.0 & 98.4 & 92.6 & 85.9 & 85.9 \\
\hline Pharmaceuticals & 78.0 & 80.3 & 78.3 & 74.8 & 71.3 & 64.9 & 71.5 & 75.9 & 80.5 & 83.8 & 93.9 & 100.0 & 110.1 & 113.1 & 90.7 & 92.3 \\
\hline Petroleum products & 114.1 & 107.4 & 100.8 & 94.8 & 90.8 & 83.5 & 69.0 & 84.4 & 88.5 & 95.1 & 98.9 & 100.0 & 103.0 & 117.4 & 107.7 & 104.4 \\
\hline Rubber and plastic products & 96.1 & 97.1 & 90.4 & 93.4 & 88.9 & 92.2 & 83.0 & 86.8 & 82.1 & 79.9 & 87.3 & 100.0 & 91.0 & 83.1 & 73.1 & 75.6 \\
\hline Stone, clay and glass & 107.0 & 108.2 & 98.9 & 90.6 & 78.3 & 85.6 & 86.5 & 82.6 & 82.8 & 87.9 & 95.5 & 100.0 & 99.3 & 98.8 & 90.8 & 90.2 \\
\hline Basic metals & 110.8 & 102.6 & 96.4 & 87.5 & 80.4 & 79.7 & 75.3 & 83.7 & 79.1 & 78.8 & 86.1 & 100.0 & 103.4 & 119.7 & 112.4 & 119.8 \\
\hline Ferrous metals & 115.9 & 110.6 & 102.8 & 86.9 & 80.0 & 80.9 & 78.2 & 83.5 & 80.3 & 81.6 & 87.1 & 100.0 & 105.3 & 121.5 & 114.7 & 126.3 \\
\hline Non-ferrous metals & 92.3 & 81.4 & 78.2 & 87.1 & 77.8 & 73.4 & 64.7 & 81.5 & 73.9 & 70.5 & 83.9 & 100.0 & 98.9 & 115.2 & 106.2 & 103.6 \\
\hline Fabricated metal products and machinery & 103.1 & 99.3 & 92.3 & 92.9 & 82.6 & 83.4 & 79.7 & 76.7 & 78.6 & 85.5 & 93.4 & 100.0 & 99.7 & 99.3 & 95.0 & 89.5 \\
\hline Fabricated metal products & 108.6 & 105.1 & 104.5 & 99.1 & 83.9 & 87.0 & 82.8 & 81.7 & 83.6 & 93.3 & 98.8 & 100.0 & 104.7 & 102.6 & 100.2 & 92.0 \\
\hline Non-electrical machinery & 133.8 & 113.0 & 86.9 & 83.0 & 80.6 & 77.2 & 68.8 & 69.5 & 75.6 & 87.2 & 88.3 & 100.0 & 97.1 & 98.9 & 88.0 & 85.0 \\
\hline Electrical machinery & 110.9 & 111.8 & 100.0 & 105.6 & 94.0 & 87.1 & 85.1 & 78.7 & 77.6 & 87.0 & 91.8 & 100.0 & 100.4 & 108.3 & 95.8 & 97.3 \\
\hline Shipbuilding & 49.3 & 73.7 & 69.0 & 68.2 & 80.2 & 126.9 & 95.8 & 89.0 & 97.1 & 69.5 & 85.8 & 100.0 & 111.1 & 87.5 & 92.3 & \\
\hline Other transport & 80.5 & 71.4 & 64.6 & 95.4 & 69.7 & 78.4 & 102.9 & 120.9 & 106.2 & 109.2 & 108.2 & 100.0 & 101.8 & 110.5 & 111.1 & 103.9 \\
\hline Motor vehicles & 109.0 & 109.3 & 121.5 & 108.2 & 90.7 & 88.6 & 87.5 & 81.8 & 84.0 & 87.7 & 100.5 & 100.0 & 94.6 & 89.3 & 93.7 & 83.5 \\
\hline Aerospace & & & & & & & & & & & & & & & .. & \\
\hline Instruments & 248.7 & 137.8 & 149.0 & 108.0 & 93.8 & 82.7 & 87.5 & 98.7 & 85.6 & 118.8 & 96.2 & 100.0 & 101.6 & 95.3 & .. & .. \\
\hline Other manufacturing industries & 192.9 & 166.7 & 112.3 & 100.0 & 80.2 & 97.2 & 86.7 & 90.1 & 91.4 & 98.7 & 105.9 & 100.0 & 99.3 & 95.0 & 69.1 & 66.5 \\
\hline
\end{tabular}

Annex Table 48: Trade-Weighted Relative Unit Labour Cost per Industry - Competitiveness among exporters

\begin{tabular}{|c|c|c|c|c|c|c|c|c|c|c|c|c|c|c|c|c|}
\hline & 1979 & 1980 & 1981 & 1982 & 1983 & 1984 & 1985 & 1986 & 1987 & 1988 & 1989 & 1990 & 1991 & 1992 & 1993 & 1994 \\
\hline Total manufacturing & 103.5 & 103.2 & 93.3 & 89.0 & 77.6 & 76.1 & 74.1 & 76.1 & 77.6 & 82.0 & 88.8 & 100.0 & 99.2 & 98.5 & 87.9 & 84.7 \\
\hline Food, drink and tobacco & 96.4 & 99.3 & 89.8 & 86.5 & 71.5 & 70.2 & 71.0 & 77.6 & 76.7 & 81.2 & 89.2 & 100.0 & 98.7 & 95.7 & 85.2 & 84.0 \\
\hline Textiles, Footwear and leather & 97.8 & 108.7 & 102.2 & 90.8 & 80.4 & 79.6 & 78.2 & 77.9 & 78.3 & 84.1 & 89.1 & 100.0 & 100.9 & 97.9 & 97.8 & 88.9 \\
\hline Wood, cork and furniture & 95.4 & 108.0 & 93.6 & 78.8 & 73.0 & 74.0 & 78.5 & 74.5 & 76.9 & 86.0 & 93.3 & 100.0 & 102.8 & 103.4 & 100.5 & 104.0 \\
\hline Paper and printing & 102.2 & 86.2 & 81.5 & 78.8 & 67.6 & 61.5 & 60.6 & 70.3 & 73.5 & 81.5 & 88.6 & 100.0 & 103.4 & 112.8 & 105.2 & 105.7 \\
\hline Chemicals & 96.5 & 94.9 & 89.4 & 87.3 & 83.0 & 80.7 & 78.3 & 82.6 & 81.7 & 81.9 & 88.2 & 100.0 & 98.0 & 95.2 & 82.4 & 81.5 \\
\hline Industrial chemicals & 95.7 & 92.6 & 88.0 & 84.4 & 78.4 & 76.0 & 77.9 & 81.3 & 82.4 & 82.9 & 89.5 & 100.0 & 98.7 & 94.0 & 84.2 & 83.4 \\
\hline Pharmaceuticals & 75.4 & 78.4 & 75.4 & 76.0 & 71.4 & 67.6 & 73.9 & 77.6 & 79.5 & 82.5 & 92.9 & 100.0 & 109.0 & 111.5 & 89.5 & 88.7 \\
\hline Petroleum products & 108.4 & 103.0 & 93.6 & 86.9 & 91.9 & 90.9 & 77.4 & 89.1 & 89.1 & 96.1 & 95.1 & 100.0 & 97.0 & 104.0 & 94.2 & 88.3 \\
\hline Rubber and plastic products & 96.8 & 97.7 & 91.2 & 92.3 & 87.3 & 88.4 & 80.0 & 84.1 & 80.7 & 77.3 & 84.0 & 100.0 & 91.0 & 85.3 & 72.9 & 73.0 \\
\hline Stone, clay and glass & 104.3 & 105.4 & 97.2 & 87.3 & 76.1 & 81.6 & 84.6 & 81.3 & 81.7 & 86.0 & 93.6 & 100.0 & 98.5 & 99.8 & 88.6 & 87.3 \\
\hline Basic metals & 114.1 & 107.2 & 98.3 & 89.7 & 78.6 & 78.9 & 75.6 & 79.7 & 78.2 & 78.0 & 82.4 & 100.0 & 102.6 & 117.3 & 104.6 & 110.0 \\
\hline Ferrous metals & 118.2 & 116.4 & 105.0 & 92.3 & 81.0 & 83.8 & 81.4 & 80.0 & 79.1 & 80.0 & 83.5 & 100.0 & 103.4 & 116.6 & 103.5 & 110.8 \\
\hline Non-ferrous metals & 97.0 & 88.1 & 80.0 & 83.9 & 70.6 & 68.4 & 61.4 & 77.4 & 74.6 & 72.1 & 79.9 & 100.0 & 100.2 & 117.4 & 105.8 & 106.2 \\
\hline Fabricated metal products and machinery & 100.2 & 99.3 & 89.4 & 89.2 & 77.4 & 76.7 & 74.1 & 72.4 & 75.9 & 82.0 & 89.2 & 100.0 & 99.0 & 99.5 & 92.0 & 85.9 \\
\hline Fabricated metal products & 107.0 & 104.7 & 103.0 & 100.5 & 84.2 & 84.9 & 81.9 & 80.6 & 82.5 & 90.6 & 95.3 & 100.0 & 104.0 & 103.3 & 99.2 & 90.2 \\
\hline Non-electrical machinery & 123.5 & 108.1 & 80.4 & 76.0 & 75.6 & 70.4 & 63.8 & 66.0 & 73.0 & 84.2 & 84.8 & 100.0 & 96.8 & 99.5 & 84.5 & 78.7 \\
\hline Electrical machinery & 91.9 & 98.5 & 83.3 & 90.5 & 83.3 & 74.0 & 75.0 & 73.1 & 72.3 & 81.9 & 86.9 & 100.0 & 99.4 & 107.7 & 94.4 & 93.6 \\
\hline Shipbuilding & 40.7 & 72.5 & 68.8 & 66.9 & 73.7 & 132.3 & 103.0 & 88.6 & 79.7 & 65.5 & 81.9 & 100.0 & 112.3 & 91.4 & 87.1 & 72.7 \\
\hline Other transport & 57.7 & 54.6 & 53.7 & 75.8 & 58.8 & 66.9 & 86.6 & 96.6 & 93.2 & 95.6 & 99.3 & 100.0 & 101.5 & 107.7 & 100.8 & 86.8 \\
\hline Motor vehicles & 116.7 & 120.1 & 129.8 & 115.7 & 91.8 & 88.9 & 86.9 & 79.5 & 83.1 & 84.4 & 95.1 & 100.0 & 92.3 & 88.9 & 92.2 & 84.1 \\
\hline Aerospace & 78.0 & 57.9 & 53.1 & 77.2 & 57.2 & 62.4 & 79.9 & 98.3 & 96.8 & 122.2 & 133.7 & 100.0 & 92.4 & 115.2 & 120.0 & 109.3 \\
\hline Instruments & 246.2 & 137.0 & 149.7 & 106.2 & 94.8 & 91.2 & 90.9 & 98.6 & 85.2 & 115.6 & 96.4 & 100.0 & 101.0 & 93.9 & 82.7 & 70.7 \\
\hline & & & & 978 & 830 & 977 & 934 & 953 & 933 & 976 & & & & & & \\
\hline
\end{tabular}

Source: OECD, DSTI (STAN Industrial Database), 1996 
DSTI/DOC(98)1

Annex Table 49: Trade-Weighted Relative Unit Labour Cost per Industry - Total price competitiveness

Sweden

\begin{tabular}{|c|c|c|c|c|c|c|c|c|c|c|c|c|c|c|c|c|}
\hline & 1979 & 1980 & 1981 & 1982 & 1983 & 1984 & 1985 & 1986 & 1987 & 1988 & 1989 & 1990 & 1991 & 1992 & 1993 & 1994 \\
\hline Total manufacturing & 107.2 & 105.1 & 106.3 & 92.1 & 81.6 & 83.7 & 87.1 & 88.4 & 87.8 & 92.8 & 99.0 & 100.0 & 102.9 & 99.9 & 73.5 & 71.2 \\
\hline Food, drink and tobacco & 92.9 & 98.2 & 99.0 & 83.6 & 78.5 & 79.2 & 81.4 & 83.5 & 81.4 & 88.1 & 97.1 & 100.0 & 104.6 & 104.6 & 81.8 & 80.7 \\
\hline Textiles, Footwear and leather & 109.1 & 112.5 & 119.7 & 109.9 & 105.2 & 102.6 & 105.8 & 101.5 & 94.0 & 102.8 & 105.8 & 100.0 & 102.3 & 95.2 & 76.0 & 73.3 \\
\hline Wood, cork and furniture & 114.0 & 118.0 & 117.1 & 96.9 & 88.0 & 93.7 & 100.0 & 98.0 & 91.9 & 97.8 & 99.2 & 100.0 & 105.4 & 113.8 & 89.2 & 86.3 \\
\hline Paper and printing & 101.2 & 100.7 & 100.4 & 88.0 & 77.5 & 86.2 & 91.3 & 90.4 & 86.8 & 91.8 & 99.4 & 100.0 & 96.4 & 91.9 & 70.1 & 69.3 \\
\hline Chemicals & 96.7 & 94.8 & 103.2 & 89.2 & 85.2 & 93.4 & 99.1 & 98.5 & 95.5 & 99.8 & 106.1 & 100.0 & 96.1 & 92.1 & 66.0 & 69.5 \\
\hline Industrial chemicals & 92.3 & 95.4 & 108.4 & 90.0 & 84.4 & 94.9 & 100.1 & 108.0 & 103.7 & 99.2 & 110.0 & 100.0 & 93.9 & 91.2 & 64.6 & 68.6 \\
\hline Pharmaceuticals & 97.3 & 80.6 & 83.2 & 72.4 & 69.8 & 76.9 & 80.5 & 94.2 & 75.7 & 76.9 & 86.7 & 100.0 & 91.9 & 81.6 & 61.9 & 60.7 \\
\hline Petroleum products & 118.8 & 109.5 & 95.0 & 91.2 & 86.3 & 98.6 & 122.0 & 97.7 & 110.1 & 115.9 & 100.9 & 100.0 & 92.6 & 104.1 & 78.6 & 87.8 \\
\hline Rubber and plastic products & 76.8 & 76.3 & 87.1 & 75.5 & 72.7 & 75.2 & 79.3 & 79.4 & 82.9 & 95.4 & 106.4 & 100.0 & 96.2 & 87.7 & 59.2 & 60.4 \\
\hline Stone, clay and glass & 120.8 & 120.8 & 128.3 & 98.9 & 86.3 & 84.8 & 94.9 & 94.3 & 90.0 & 90.8 & 97.3 & 100.0 & 109.9 & 118.0 & 92.2 & 91.2 \\
\hline Basic metals & 122.5 & 116.4 & 124.8 & 101.9 & 90.5 & 83.0 & 91.5 & 94.9 & 89.5 & 94.3 & 102.5 & 100.0 & 100.1 & 95.8 & 67.6 & 66.0 \\
\hline Ferrous metals & 118.6 & 116.5 & 125.7 & 98.7 & 87.6 & 76.5 & 84.1 & 87.0 & 85.9 & 89.1 & 98.9 & 100.0 & 99.0 & 93.0 & 65.7 & 63.0 \\
\hline Non-ferrous metals & 135.4 & 121.1 & 127.1 & 112.3 & 102.6 & 107.1 & 118.0 & 122.0 & 100.1 & 111.6 & 113.8 & 100.0 & 102.9 & 103.9 & 71.9 & 75.5 \\
\hline Fabricated metal products and machinery & 107.8 & 103.7 & 100.0 & 89.3 & 76.4 & 76.9 & 79.0 & 82.3 & 86.3 & 92.0 & 97.5 & 100.0 & 106.2 & 102.4 & 74.1 & 69.5 \\
\hline Fabricated metal products & 120.4 & 113.7 & 118.3 & 105.7 & 92.6 & 95.7 & 95.4 & 91.7 & 89.3 & 94.9 & 102.2 & 100.0 & 104.5 & 103.2 & 77.2 & 67.3 \\
\hline Non-electrical machinery & 102.7 & 98.2 & 98.0 & 86.0 & 71.2 & 70.6 & 75.1 & 77.8 & 82.4 & 88.8 & 92.9 & 100.0 & 106.9 & 101.4 & 76.4 & 71.4 \\
\hline Electrical machinery & 144.6 & 124.6 & 114.2 & 115.0 & 103.9 & 97.3 & 78.2 & 74.1 & 81.2 & 90.8 & 109.5 & 100.0 & 94.1 & 87.0 & 50.4 & 39.0 \\
\hline Shipbuilding & 44.5 & 66.3 & 61.4 & 46.3 & 42.1 & 47.7 & 47.3 & 56.9 & 70.3 & 95.0 & 108.4 & 100.0 & 114.9 & 117.5 & .. & \\
\hline Other transport & 58.1 & 68.0 & 70.0 & 65.4 & 65.4 & 63.7 & 77.2 & 76.2 & 77.5 & 88.4 & 90.1 & 100.0 & 103.5 & 105.8 & 63.9 & 62.4 \\
\hline Motor vehicles & 111.0 & 104.1 & 94.5 & 81.2 & 66.2 & 68.7 & 78.0 & 77.0 & 75.1 & 81.6 & 90.5 & 100.0 & 107.5 & 103.3 & 74.8 & 77.1 \\
\hline Aerospace & 91.7 & 90.0 & 70.2 & 66.8 & 55.5 & 54.4 & 56.1 & 66.2 & 81.1 & 93.4 & 86.3 & 100.0 & 111.9 & 105.4 & 88.5 & 77.1 \\
\hline Instruments & 119.2 & 99.9 & 99.8 & 89.2 & 76.9 & & 80.3 & 90.6 & 106.6 & 114.8 & 105.4 & 100.0 & 104.1 & 106.5 & 79.7 & 73.6 \\
\hline Other manufacturing industries & 129.4 & 205.8 & 178.7 & 119.7 & 127.9 & 100.4 & 83.3 & 83.3 & 85.1 & 90.9 & 91.6 & 100.0 & 112.3 & 106.1 & 80.2 & 81.8 \\
\hline
\end{tabular}

Annex Table 50: Trade-Weighted Relative Unit Labour Cost per Industry - Competitiveness among exporters

Sweden

\begin{tabular}{|c|c|c|c|c|c|c|c|c|c|c|c|c|c|c|c|c|}
\hline & 1979 & 1980 & 1981 & 1982 & 1983 & 1984 & 1985 & 1986 & 1987 & 1988 & 1989 & 1990 & 1991 & 1992 & 1993 & 1994 \\
\hline Total manufacturing & 104.4 & 103.1 & 103.2 & 89.0 & 77.6 & 79.0 & 83.6 & 85.0 & 85.9 & 91.1 & 96.6 & 100.0 & 101.6 & 97.6 & 69.5 & 67.1 \\
\hline Food, drink and tobacco & 91.7 & 94.5 & 94.7 & 80.2 & 72.6 & 73.6 & 77.6 & 81.6 & 80.4 & 87.4 & 95.9 & 100.0 & 101.9 & 100.3 & 77.1 & 77.0 \\
\hline Textiles, Footwear and leather & 101.5 & 106.3 & 115.6 & 106.9 & 100.4 & 96.8 & 101.1 & 97.1 & 91.0 & 100.6 & 104.4 & 100.0 & 101.5 & 94.3 & 73.6 & 71.5 \\
\hline Wood, cork and furniture & 102.0 & 109.9 & 111.2 & 88.1 & 78.0 & 82.6 & 91.1 & 93.1 & 90.0 & 96.3 & 96.0 & 100.0 & 105.4 & 111.0 & 83.7 & 82.6 \\
\hline Paper and printing & 102.1 & 102.8 & 99.2 & 84.2 & 72.7 & 79.4 & 84.7 & 87.6 & 86.7 & 92.4 & 97.4 & 100.0 & 95.1 & 90.7 & 67.9 & 66.6 \\
\hline Chemicals & 97.0 & 91.4 & 100.9 & 86.7 & 81.6 & 87.5 & 95.0 & 96.0 & 94.5 & 99.2 & 104.9 & 100.0 & 96.3 & 91.2 & 63.9 & 66.7 \\
\hline Industrial chemicals & 90.3 & 91.3 & 104.8 & 88.0 & 82.7 & 88.9 & 97.2 & 104.5 & 102.9 & 98.0 & 108.2 & 100.0 & 94.0 & 91.4 & 63.3 & 67.0 \\
\hline Pharmaceuticals & 94.1 & 76.2 & 78.1 & 66.6 & 67.2 & 76.1 & 81.5 & 94.3 & 75.9 & 78.8 & 87.6 & 100.0 & 91.9 & 81.7 & 61.3 & 59.1 \\
\hline Petroleum products & 119.4 & 107.5 & 95.1 & 86.8 & 78.2 & 86.1 & 100.8 & 90.0 & 100.0 & 113.1 & 103.1 & 100.0 & 98.8 & 103.5 & 76.7 & 83.1 \\
\hline Rubber and plastic products & 80.1 & 75.0 & 86.9 & 74.7 & 71.0 & 73.5 & 77.7 & 78.3 & 83.2 & 95.1 & 104.7 & 100.0 & 95.6 & 85.6 & 56.2 & 57.5 \\
\hline Stone, clay and glass & 115.6 & 115.3 & 121.5 & 92.5 & 81.2 & 79.5 & 91.7 & 91.9 & 88.7 & 91.4 & 95.6 & 100.0 & 110.2 & 114.7 & 87.8 & 88.1 \\
\hline Basic metals & 124.7 & 122.6 & 129.8 & 103.6 & 87.6 & 82.8 & 92.2 & 91.7 & 87.3 & 93.2 & 101.3 & 100.0 & 98.0 & 90.8 & 62.2 & 60.7 \\
\hline Ferrous metals & 120.9 & 124.9 & 132.9 & 102.9 & 85.3 & 78.6 & 86.5 & 83.1 & 83.5 & 87.2 & 97.3 & 100.0 & 95.4 & 85.4 & 58.4 & 55.7 \\
\hline Non-ferrous metals & 137.4 & 126.4 & 128.4 & 111.1 & 98.4 & 102.9 & 116.6 & 122.3 & 99.6 & 113.1 & 112.7 & 100.0 & 104.6 & 104.3 & 71.2 & 76.0 \\
\hline Fabricated metal products and machinery & 103.1 & 101.3 & 95.7 & 85.5 & 72.5 & 73.2 & 76.4 & 78.9 & 83.5 & 89.3 & 94.6 & 100.0 & 105.4 & 100.5 & 70.6 & 66.1 \\
\hline Fabricated metal products & 114.1 & 107.1 & 110.9 & 98.9 & 86.4 & 88.1 & 90.0 & 87.7 & 87.4 & 93.4 & 100.2 & 100.0 & 102.5 & 100.4 & 74.1 & 65.6 \\
\hline Non-electrical machinery & 95.8 & 93.2 & 90.9 & 79.0 & 66.4 & 66.4 & 71.1 & 75.3 & 80.5 & 87.3 & 90.7 & 100.0 & 106.7 & 100.1 & 72.9 & 67.4 \\
\hline Electrical machinery & 122.3 & 110.2 & 99.8 & 103.5 & 95.3 & 90.8 & 74.7 & 69.3 & 76.5 & 87.4 & 105.2 & 100.0 & 93.1 & 83.0 & 47.3 & 36.8 \\
\hline Shipbuilding & 43.3 & 62.0 & 54.5 & 45.7 & 39.1 & 48.7 & 49.6 & 51.5 & 64.1 & 92.1 & 106.5 & 100.0 & 114.2 & 114.7 & 76.7 & 73.6 \\
\hline Other transport & 41.4 & 53.7 & 56.6 & 56.4 & 57.6 & 59.6 & 70.5 & 64.0 & 71.5 & 81.1 & 84.2 & 100.0 & 99.0 & 97.9 & 56.9 & 58.1 \\
\hline Motor vehicles & 104.4 & 104.5 & 94.2 & 81.7 & 64.6 & 65.7 & 75.7 & 73.5 & 71.5 & 78.9 & 88.9 & 100.0 & 106.7 & 101.8 & 73.6 & 74.4 \\
\hline Aerospace & 87.3 & 87.4 & 75.6 & 74.5 & 64.5 & 65.3 & 67.3 & 73.9 & 80.6 & 89.9 & 89.9 & 100.0 & 112.5 & 105.7 & 89.4 & 77.6 \\
\hline Instruments & 118.6 & 98.4 & 100.7 & 90.2 & 81.7 & 82.5 & 88.0 & 94.4 & 107.8 & 113.7 & 107.4 & 100.0 & 103.7 & 105.0 & 77.4 & 72.9 \\
\hline & & & 1782 & & & & 912 & 868 & & & & & & & & \\
\hline
\end{tabular}

Source: OECD, DSTI (STAN Industrial Database), 1996. 


\begin{tabular}{|c|c|c|c|c|c|c|c|c|c|c|c|c|c|c|c|c|}
\hline & 1979 & 1980 & 1981 & 1982 & 1983 & 1984 & 1985 & 1986 & 1987 & 1988 & 1989 & 1990 & 1991 & 1992 & 1993 & 1994 \\
\hline Total manufacturing & 93.5 & 114.0 & 120.5 & 113.5 & 103.7 & 99.0 & 102.1 & 96.6 & 97.1 & 103.8 & 98.8 & 100.0 & 102.5 & 95.6 & 85.0 & 85.4 \\
\hline Food, drink and tobacco & 70.9 & 87.2 & 98.3 & 94.4 & 88.1 & 87.5 & 92.0 & 87.4 & 82.2 & 92.2 & 102.4 & 100.0 & 106.7 & 97.8 & 92.4 & 94.4 \\
\hline Textiles, Footwear and leather & 81.7 & 102.1 & 106.8 & 103.6 & 94.3 & 91.7 & 95.3 & 91.2 & 86.6 & 96.8 & 97.8 & 100.0 & 104.9 & 107.4 & 91.9 & 94.2 \\
\hline Wood, cork and furniture & 74.1 & 101.1 & 106.1 & 108.6 & 112.5 & 104.0 & 110.9 & 103.1 & 103.8 & 108.8 & 102.8 & 100.0 & 102.9 & 97.6 & 96.0 & 94.8 \\
\hline Paper and printing & 93.2 & 116.9 & 122.7 & 121.2 & 123.1 & 119.9 & 123.4 & 116.4 & 111.2 & 112.4 & 101.0 & 100.0 & 107.6 & 108.3 & 100.1 & 102.4 \\
\hline Chemicals & 89.7 & 109.5 & 118.4 & 111.4 & 103.0 & 97.4 & 97.9 & 92.3 & 96.2 & 103.9 & 100.3 & 100.0 & 98.5 & 92.8 & 84.2 & 85.2 \\
\hline Industrial chemicals & 89.1 & 108.0 & 115.4 & 107.0 & 95.8 & 89.9 & 91.4 & 82.7 & 91.3 & 98.6 & 94.8 & 100.0 & 95.3 & 88.7 & 80.5 & 81.1 \\
\hline Pharmaceuticals & 82.1 & 99.8 & 113.6 & 104.7 & 103.4 & 97.9 & 105.8 & 99.0 & 100.8 & 108.2 & 106.1 & 100.0 & 104.6 & 98.4 & 85.6 & 86.9 \\
\hline Petroleum products & 103.6 & 133.4 & 120.1 & 112.6 & 111.7 & 102.2 & 101.3 & 92.4 & 92.9 & 102.4 & 100.5 & 100.0 & 88.9 & 93.1 & 86.4 & 81.1 \\
\hline Rubber and plastic products & 82.2 & 102.9 & 112.0 & 108.7 & 99.9 & 98.8 & 100.8 & 104.0 & 104.3 & 111.9 & 107.8 & 100.0 & 102.4 & 96.2 & 86.0 & 85.2 \\
\hline Stone, clay and glass & 83.9 & 102.5 & 109.5 & 102.6 & 101.5 & 96.4 & 101.8 & 98.1 & 98.4 & 104.9 & 105.7 & 100.0 & 97.1 & 91.4 & 84.3 & 82.5 \\
\hline Basic metals & 118.8 & 158.4 & 139.3 & 116.3 & 99.5 & 97.5 & 104.0 & 99.3 & 97.2 & 103.9 & 98.9 & 100.0 & 103.6 & 99.0 & 95.0 & 94.9 \\
\hline Ferrous metals & 122.3 & 168.6 & 147.8 & 122.5 & 109.0 & 108.1 & 112.3 & 103.2 & 97.9 & 105.8 & 102.7 & 100.0 & 103.6 & 98.8 & 96.0 & 98.6 \\
\hline Non-ferrous metals & 113.9 & 138.6 & 137.6 & 125.3 & 96.3 & 88.4 & 95.7 & 93.1 & 97.2 & 104.7 & 94.5 & 100.0 & 105.6 & 99.6 & 94.0 & 89.7 \\
\hline Fabricated metal products and machinery & 97.6 & 118.7 & 123.1 & 114.5 & 103.4 & 97.4 & 100.2 & 95.5 & 99.8 & 105.8 & 96.0 & 100.0 & 103.4 & 94.2 & 82.3 & 82.7 \\
\hline Fabricated metal products & 81.8 & 104.4 & 106.0 & 98.9 & 93.9 & 94.3 & 101.4 & 106.7 & 101.2 & 104.6 & 97.1 & 100.0 & 107.3 & 102.8 & 92.5 & 90.1 \\
\hline Non-electrical machinery & 87.3 & 107.3 & 108.4 & 97.5 & 93.8 & 90.8 & 95.3 & 93.4 & 99.1 & 106.7 & 100.2 & 100.0 & 106.2 & 105.9 & 93.7 & 90.3 \\
\hline Electrical machinery & 90.9 & 114.7 & 117.5 & 112.0 & 101.6 & 99.3 & 108.0 & 103.5 & 110.8 & 114.5 & 103.2 & 100.0 & 109.3 & 101.0 & 81.9 & 83.7 \\
\hline Shipbuilding & & & & & & & & & & .. & .. & .. & .. & .. & & \\
\hline Other transport & 136.8 & 155.8 & 169.7 & 185.0 & 159.7 & 139.5 & 140.9 & 104.4 & 102.9 & 97.2 & 86.7 & 100.0 & 91.1 & 70.8 & 61.8 & 70.5 \\
\hline Motor vehicles & 98.3 & 113.5 & 130.2 & 120.4 & 106.1 & 98.5 & 99.8 & 90.9 & 89.5 & 92.9 & 92.7 & 100.0 & 96.3 & 78.3 & 71.8 & 73.8 \\
\hline Aerospace & 157.9 & 160.8 & 129.8 & 147.0 & 125.8 & 114.3 & 104.1 & 86.9 & 113.7 & 135.1 & 92.4 & 100.0 & 94.8 & 79.0 & 73.3 & 72.1 \\
\hline Instruments & & & & & .. & .. & .. & .. & .. & .. & .. & .. & .. & .. & .. & \\
\hline
\end{tabular}

Annex Table 52: Trade-Weighted Relative Unit Labour Cost per Industry - Competitiveness among exporters

United Kingdom

\begin{tabular}{|c|c|c|c|c|c|c|c|c|c|c|c|c|c|c|c|c|}
\hline & 1979 & 1980 & 1981 & 1982 & 1983 & 1984 & 1985 & 1986 & 1987 & 1988 & 1989 & 1990 & 1991 & 1992 & 1993 & 1994 \\
\hline Total manufacturing & 95.1 & 116.5 & 120.6 & 112.7 & 101.6 & 96.5 & 99.6 & 94.3 & 96.0 & 101.9 & 96.8 & 100.0 & 101.9 & 95.3 & 83.0 & 82.8 \\
\hline Food, drink and tobacco & 74.3 & 89.9 & 97.7 & 93.2 & 84.1 & 81.6 & 86.2 & 85.5 & 82.7 & 93.1 & 100.5 & 100.0 & 104.7 & 96.8 & 90.6 & 92.7 \\
\hline Textiles, Footwear and leather & 80.3 & 101.5 & 107.2 & 102.2 & 92.5 & 89.1 & 93.5 & 90.5 & 86.2 & 96.2 & 96.9 & 100.0 & 105.1 & 107.6 & 90.8 & 93.3 \\
\hline Wood, cork and furniture & 71.9 & 99.7 & 104.7 & 103.7 & 103.6 & 96.2 & 103.6 & 100.7 & 102.1 & 107.3 & 101.1 & 100.0 & 103.7 & 98.1 & 91.5 & 91.1 \\
\hline Paper and printing & 92.2 & 116.0 & 121.4 & 118.2 & 117.3 & 113.3 & 115.7 & 112.9 & 109.5 & 110.3 & 98.8 & 100.0 & 106.3 & 106.5 & 94.8 & 97.4 \\
\hline Chemicals & 92.5 & 112.4 & 118.6 & 109.3 & 100.0 & 93.7 & 94.6 & 90.5 & 96.2 & 102.7 & 98.8 & 100.0 & 98.1 & 93.0 & 82.8 & 82.9 \\
\hline Industrial chemicals & 91.5 & 110.1 & 115.5 & 107.3 & 95.7 & 88.6 & 89.4 & 81.9 & 91.7 & 98.3 & 94.3 & 100.0 & 95.4 & 89.1 & 80.2 & 80.7 \\
\hline Pharmaceuticals & 80.4 & 96.3 & 112.1 & 104.5 & 103.6 & 99.0 & 106.3 & 98.7 & 100.8 & 107.7 & 105.4 & 100.0 & 104.3 & 97.7 & 84.5 & 84.0 \\
\hline Petroleum products & 112.2 & 143.8 & 137.6 & 115.4 & 111.7 & 98.1 & 96.8 & 92.9 & 97.5 & 105.4 & 101.3 & 100.0 & 93.3 & 96.7 & 84.2 & 82.5 \\
\hline Rubber and plastic products & 86.1 & 106.4 & 114.4 & 109.2 & 98.5 & 95.7 & 98.2 & 102.0 & 104.1 & 109.8 & 105.6 & 100.0 & 101.8 & 96.4 & 84.8 & 83.4 \\
\hline Stone, clay and glass & 86.7 & 106.4 & 111.9 & 104.2 & 102.4 & 95.1 & 101.3 & 96.8 & 97.9 & 103.2 & 103.5 & 100.0 & 96.5 & 91.2 & 82.9 & 81.3 \\
\hline Basic metals & 123.3 & 162.0 & 148.7 & 124.7 & 101.2 & 100.0 & 105.5 & 95.5 & 94.5 & 102.5 & 98.6 & 100.0 & 102.2 & 96.0 & 88.6 & 88.1 \\
\hline Ferrous metals & 127.8 & 178.2 & 155.4 & 126.9 & 104.2 & 106.2 & 110.9 & 97.1 & 94.5 & 102.1 & 100.4 & 100.0 & 100.9 & 94.2 & 86.6 & 87.3 \\
\hline Non-ferrous metals & 114.2 & 135.3 & 137.4 & 126.0 & 95.2 & 89.1 & 96.7 & 91.9 & 96.2 & 106.4 & 95.8 & 100.0 & 106.5 & 100.1 & 93.4 & 91.1 \\
\hline Fabricated metal products and machinery & 96.5 & 119.6 & 121.8 & 113.0 & 100.9 & 95.1 & 98.7 & 93.1 & 97.9 & 102.9 & 93.7 & 100.0 & 102.8 & 93.9 & 80.6 & 80.2 \\
\hline Fabricated metal products & 82.1 & 105.4 & 104.9 & 99.6 & 92.2 & 91.1 & 99.7 & 105.3 & 100.4 & 103.0 & 95.1 & 100.0 & 106.7 & 102.9 & 92.1 & 89.1 \\
\hline Non-electrical machinery & 86.0 & 107.2 & 108.4 & 97.8 & 94.1 & 91.6 & 97.3 & 94.0 & 98.9 & 105.2 & 98.9 & 100.0 & 105.9 & 105.6 & 90.6 & 86.4 \\
\hline Electrical machinery & 80.8 & 106.5 & 109.1 & 106.5 & 98.3 & 97.0 & 105.8 & 100.7 & 107.3 & 111.1 & 100.8 & 100.0 & 108.4 & 99.4 & 80.0 & 80.9 \\
\hline Shipbuilding & 58.9 & 84.6 & 89.4 & 85.3 & 71.1 & 72.3 & 75.8 & 68.5 & 70.7 & 83.6 & 79.1 & 100.0 & 86.3 & 84.2 & 75.9 & 68.6 \\
\hline Other transport & 91.9 & 124.8 & 140.1 & 151.6 & 137.6 & 127.4 & 125.8 & 91.6 & 95.2 & 88.3 & 80.4 & 100.0 & 90.7 & 68.8 & 58.7 & 59.4 \\
\hline Motor vehicles & 97.8 & 116.1 & 128.0 & 116.6 & 99.2 & 91.0 & 90.4 & 85.2 & 87.0 & 90.4 & 89.9 & 100.0 & 94.1 & 77.7 & 71.7 & 72.7 \\
\hline Aerospace & 156.4 & 159.9 & 136.5 & 154.9 & 136.6 & 127.5 & 119.3 & 90.6 & 112.5 & 132.7 & 94.6 & 100.0 & 95.9 & 78.9 & 74.4 & 75.4 \\
\hline Instruments & 88.6 & 111.9 & 107.5 & 110.9 & 92.9 & 83.3 & 87.5 & 87.5 & 90.5 & 100.5 & 94.4 & 100.0 & 101.0 & 92.8 & 71.5 & 68.3 \\
\hline Other manufacturing industries & 75.7 & 88.0 & 99.7 & 101.3 & 85.9 & 103.6 & 107.7 & 96.7 & 103.1 & 110.3 & 103.8 & 100.0 & 107.0 & 103.4 & 83.0 & 78.0 \\
\hline
\end{tabular}

Source: OECD, DSTI (STAN Industrial Database), 1996. 
DSTI/DOC(98)1

Annex Table 53: Trade-Weighted Relative Unit Labour Cost per Industry - Total price competitiveness

United States

\begin{tabular}{|c|c|c|c|c|c|c|c|c|c|c|c|c|c|c|c|c|}
\hline & 1979 & 1980 & 1981 & 1982 & 1983 & 1984 & 1985 & 1986 & 1987 & 1988 & 1989 & 1990 & 1991 & 1992 & 1993 & 1994 \\
\hline Total manufacturing & 120.6 & 121.8 & 129.4 & 139.4 & 138.2 & 146.5 & 152.9 & 123.7 & 104.4 & 96.6 & 102.6 & 100.0 & 98.3 & 96.5 & 95.8 & 92.7 \\
\hline Food, drink and tobacco & 93.4 & 93.4 & 97.7 & 112.2 & 121.7 & 133.7 & 136.9 & 121.7 & 107.5 & 96.5 & 104.5 & 100.0 & 105.7 & 109.1 & 122.1 & 119.4 \\
\hline Textiles, Footwear and leather & 133.9 & 128.4 & 152.4 & 167.4 & 164.9 & 174.5 & 176.9 & 135.8 & 118.8 & 112.7 & 115.1 & 100.0 & 97.1 & 92.1 & 103.5 & 103.4 \\
\hline Wood, cork and furniture & 114.2 & 109.6 & 116.3 & 108.2 & 112.0 & 123.3 & 136.9 & 127.1 & 111.4 & 106.5 & 103.9 & 100.0 & 97.9 & 111.1 & 130.8 & 130.9 \\
\hline Paper and printing & 109.4 & 113.6 & 113.5 & 106.4 & 111.6 & 123.9 & 126.3 & 121.5 & 114.0 & 104.7 & 101.3 & 100.0 & 95.6 & 101.1 & 111.5 & 120.5 \\
\hline Chemicals & 126.3 & 130.5 & 135.7 & 144.1 & 143.0 & 159.6 & 161.0 & 130.0 & 100.8 & 97.5 & 104.2 & 100.0 & 97.5 & 99.6 & 104.8 & 104.0 \\
\hline Industrial chemicals & 129.7 & 133.8 & 142.3 & 155.8 & 151.6 & 168.6 & 167.8 & 131.9 & 98.3 & 94.4 & 102.3 & 100.0 & 99.4 & 98.1 & 101.1 & 98.2 \\
\hline Pharmaceuticals & 94.4 & 108.2 & 120.3 & 125.9 & 134.7 & 151.2 & 152.3 & 120.2 & 92.2 & 90.7 & 109.0 & 100.0 & 99.0 & 98.0 & 107.9 & 112.8 \\
\hline Petroleum products & 128.5 & 134.7 & 117.6 & 118.9 & 119.7 & 140.2 & 135.7 & 127.0 & 93.6 & 91.8 & 91.9 & 100.0 & 106.1 & 112.3 & 124.6 & 133.5 \\
\hline Rubber and plastic products & 133.7 & 133.5 & 140.3 & 153.1 & 142.7 & 154.8 & 163.9 & 129.6 & 108.2 & 103.6 & 107.0 & 100.0 & 93.5 & 97.6 & 99.0 & 95.6 \\
\hline Stone, clay and glass & 128.0 & 126.6 & 144.9 & 169.0 & 157.4 & 164.9 & 170.3 & 129.9 & 120.0 & 113.4 & 111.9 & 100.0 & 98.0 & 89.7 & 96.2 & 97.9 \\
\hline Basic metals & 113.8 & 105.1 & 109.3 & 117.7 & 129.4 & 132.8 & 141.1 & 100.2 & 101.3 & 99.9 & 105.5 & 100.0 & 93.0 & 95.6 & 100.2 & 107.6 \\
\hline Ferrous metals & 126.6 & 124.2 & 120.6 & 139.6 & 144.1 & 149.4 & 157.3 & 107.9 & 102.3 & 101.3 & 108.5 & 100.0 & 93.4 & 94.6 & 97.8 & 104.7 \\
\hline Non-ferrous metals & 83.2 & 77.1 & 78.0 & 81.8 & 105.4 & 106.5 & 116.7 & 88.9 & 100.7 & 98.0 & 101.1 & 100.0 & 92.9 & 96.3 & 102.9 & 111.8 \\
\hline Fabricated metal products and machinery & 110.3 & 120.2 & 127.2 & 138.6 & 133.0 & 137.2 & 150.1 & 123.1 & 101.0 & 92.5 & 101.0 & 100.0 & 100.3 & 95.8 & 90.5 & 85.8 \\
\hline Fabricated metal products & 121.0 & 118.8 & 127.2 & 144.9 & 137.9 & 139.9 & 152.6 & 125.2 & 105.3 & 96.5 & 101.6 & 100.0 & 97.3 & 94.5 & 95.4 & 90.9 \\
\hline Non-electric & 163.5 & 159.5 & 168.0 & 184.8 & 167.3 & 179.9 & 190.8 & 147.2 & 117.7 & 100.6 & 106.4 & 100.0 & 101.8 & 91.2 & 86.2 & 79.8 \\
\hline Electrical machinery & 100.3 & 110.9 & 113.0 & 137.8 & 131.7 & 142.2 & 158.3 & 128.5 & 108.4 & 99.3 & 104.4 & 100.0 & 97.6 & 89.2 & 78.9 & 69.3 \\
\hline Shipbuilding & 65.0 & 67.3 & 82.7 & 61.2 & 84.4 & 103.9 & 103.7 & 99.4 & 59.5 & 77.1 & 90.4 & 100.0 & 112.7 & 128.9 & 154.3 & 179.2 \\
\hline Other transport & 181.6 & 163.0 & 192.1 & 135.9 & 144.8 & 173.0 & 148.9 & 105.5 & 72.5 & 75.5 & 88.8 & 100.0 & 104.6 & 125.4 & 145.7 & 206.1 \\
\hline Motor vehicles & 92.0 & 118.7 & 109.5 & 118.4 & 102.0 & 94.4 & 104.8 & 94.2 & 83.7 & 82.1 & 93.8 & 100.0 & 108.1 & 102.0 & 98.5 & 92.6 \\
\hline Aerospace & 82.3 & 78.9 & 114.4 & 100.7 & 120.8 & 133.4 & 138.5 & 128.5 & 99.0 & 92.9 & 111.1 & 100.0 & 97.6 & 106.9 & 110.6 & 125.3 \\
\hline Instruments & 94.0 & 110.2 & 123.3 & 152.4 & 157.3 & 160.9 & 180.0 & 136.1 & 101.3 & 88.6 & 107.5 & 100.0 & 102.0 & 95.7 & 90.9 & 84.1 \\
\hline Other manufact & 173.6 & 167.1 & 158.9 & 185.3 & 187.2 & 181.9 & 181.8 & 134.8 & 116.6 & 105.1 & 112.2 & 100.0 & 98.9 & 103.6 & 101.2 & 97. \\
\hline
\end{tabular}

Annex Table 54: Trade-Weighted Relative Unit Labour Cost per Industry - Competitiveness among exporters

United States

\begin{tabular}{|c|c|c|c|c|c|c|c|c|c|c|c|c|c|c|c|c|}
\hline & 1979 & 1980 & 1981 & 1982 & 1983 & 1984 & 1985 & 1986 & 1987 & 1988 & 1989 & 1990 & 1991 & 1992 & 1993 & 1994 \\
\hline Total manufacturing & 112.1 & 113.0 & 130.4 & 148.0 & 152.6 & 162.1 & 167.6 & 129.9 & 105.3 & 100.2 & 109.2 & 100.0 & 100.5 & 95.6 & 97.8 & 95.0 \\
\hline Food, drink and tobacco & 86.5 & 87.5 & 99.3 & 115.9 & 130.2 & 142.5 & 144.5 & 122.6 & 103.2 & 95.5 & 108.4 & 100.0 & 106.9 & 106.6 & 118.5 & 113.1 \\
\hline Textiles, Footwear and leather & 125.5 & 121.5 & 147.8 & 164.5 & 165.4 & 175.1 & 178.5 & 134.6 & 115.5 & 110.7 & 115.5 & 100.0 & 97.7 & 90.2 & 100.6 & 98.3 \\
\hline Wood, cork and furniture & 119.0 & 110.0 & 127.7 & 133.5 & 141.5 & 149.4 & 157.4 & 128.6 & 108.1 & 106.9 & 110.2 & 100.0 & 99.5 & 103.4 & 125.6 & 125.3 \\
\hline Paper and printing & 90.1 & 93.7 & 107.4 & 117.1 & 128.6 & 143.2 & 145.5 & 121.4 & 108.0 & 104.6 & 107.7 & 100.0 & 98.8 & 97.7 & 110.9 & 116.3 \\
\hline Chemicals & 117.5 & 122.3 & 140.1 & 161.5 & 162.0 & 181.9 & 179.0 & 137.5 & 100.1 & 100.0 & 109.5 & 100.0 & 99.7 & 97.4 & 104.4 & 103.2 \\
\hline Industrial chemicals & 120.6 & 126.0 & 146.8 & 172.8 & 168.8 & 190.9 & 188.2 & 141.3 & 99.5 & 97.5 & 107.6 & 100.0 & 101.6 & 96.5 & 102.5 & 100.0 \\
\hline Pharmaceuticals & 83.0 & 96.9 & 110.2 & 120.9 & 135.7 & 154.5 & 157.8 & 125.8 & 91.8 & 93.7 & 113.4 & 100.0 & 100.8 & 98.7 & 110.3 & 115.8 \\
\hline Petroleum products & 111.7 & 113.7 & 124.1 & 136.9 & 147.0 & 162.1 & 149.2 & 128.7 & 89.0 & 92.9 & 100.6 & 100.0 & 103.3 & 104.7 & 115.6 & 116.7 \\
\hline Rubber and plastic products & 124.0 & 127.0 & 140.6 & 164.5 & 156.3 & 170.6 & 177.5 & 134.5 & 106.2 & 107.5 & 113.1 & 100.0 & 95.8 & 92.4 & 93.5 & 89.5 \\
\hline Stone, clay and glass & 116.6 & 116.0 & 142.4 & 174.5 & 164.9 & 175.3 & 176.0 & 131.4 & 118.3 & 114.4 & 115.7 & 100.0 & 100.3 & 89.0 & 97.1 & 97.6 \\
\hline Basic metals & 98.4 & 91.8 & 104.2 & 123.2 & 141.4 & 140.7 & 149.6 & 102.5 & 97.7 & 100.0 & 111.3 & 100.0 & 94.2 & 91.8 & 91.3 & 97.1 \\
\hline Ferrous metals & 106.0 & 106.1 & 115.0 & 144.1 & 150.7 & 155.5 & 164.9 & 110.4 & 101.1 & 103.4 & 112.9 & 100.0 & 96.7 & 92.6 & 91.5 & 100.1 \\
\hline Non-ferrous metals & 84.9 & 74.2 & 82.6 & 94.2 & 126.9 & 121.8 & 129.0 & 92.0 & 94.0 & 95.6 & 109.2 & 100.0 & 89.9 & 90.0 & 90.8 & 90.5 \\
\hline Fabricated metal products and machinery & 112.1 & 118.1 & 133.9 & 152.4 & 151.4 & 153.8 & 166.9 & 131.0 & 104.1 & 96.7 & 107.6 & 100.0 & 102.4 & 94.9 & 91.4 & 86.8 \\
\hline Fabricated metal products & 118.5 & 115.0 & 129.5 & 151.3 & 151.9 & 157.0 & 166.1 & 126.9 & 103.0 & 99.0 & 108.1 & 100.0 & 100.9 & 93.3 & 96.2 & 91.0 \\
\hline Non-electrical machinery & 173.6 & 165.2 & 183.6 & 212.0 & 194.4 & 200.8 & 206.5 & 153.3 & 120.4 & 105.1 & 112.9 & 100.0 & 102.4 & 90.1 & 89.3 & 82.2 \\
\hline Electrical machinery & 125.5 & 124.5 & 133.2 & 160.9 & 158.2 & 166.9 & 180.0 & 143.7 & 117.3 & 107.7 & 112.2 & 100.0 & 98.5 & 87.5 & 79.7 & 71.6 \\
\hline Shipbuilding & 65.2 & 73.2 & 92.7 & 79.1 & 88.9 & 106.3 & 113.1 & 99.2 & 73.3 & 78.0 & 98.3 & 100.0 & 107.7 & 122.9 & 134.1 & 147.9 \\
\hline Other transport & 135.0 & 156.1 & 175.4 & 128.4 & 120.4 & 151.5 & 141.7 & 96.3 & 71.8 & 72.0 & 91.8 & 100.0 & 105.3 & 108.9 & 118.8 & 165.8 \\
\hline Motor vehicles & 87.4 & 109.6 & 112.3 & 129.2 & 119.8 & 108.0 & 121.3 & 100.1 & 86.5 & 84.2 & 99.2 & 100.0 & 111.9 & 101.8 & 95.9 & 90.7 \\
\hline Aerospace & 82.0 & 81.2 & 126.7 & 110.4 & 129.3 & 137.0 & 141.9 & 127.4 & 97.7 & 91.6 & 111.2 & 100.0 & 101.0 & 104.3 & 107.2 & 119.6 \\
\hline Instruments & 98.0 & 106.0 & 129.6 & 155.2 & 172.6 & 180.9 & 192.6 & 147.5 & 109.7 & 96.3 & 115.1 & 100.0 & 102.4 & 96.9 & 99.4 & 96.1 \\
\hline Other $m$ & 134.5 & 150.6 & 158.1 & 192.9 & 211.9 & 201.9 & 196.5 & 145.6 & 117.3 & 108.4 & 111.0 & 100.0 & 103.5 & 106.0 & 105.5 & \\
\hline
\end{tabular}

Source: OECD, DSTI (STAN Industrial Database), 1996. 


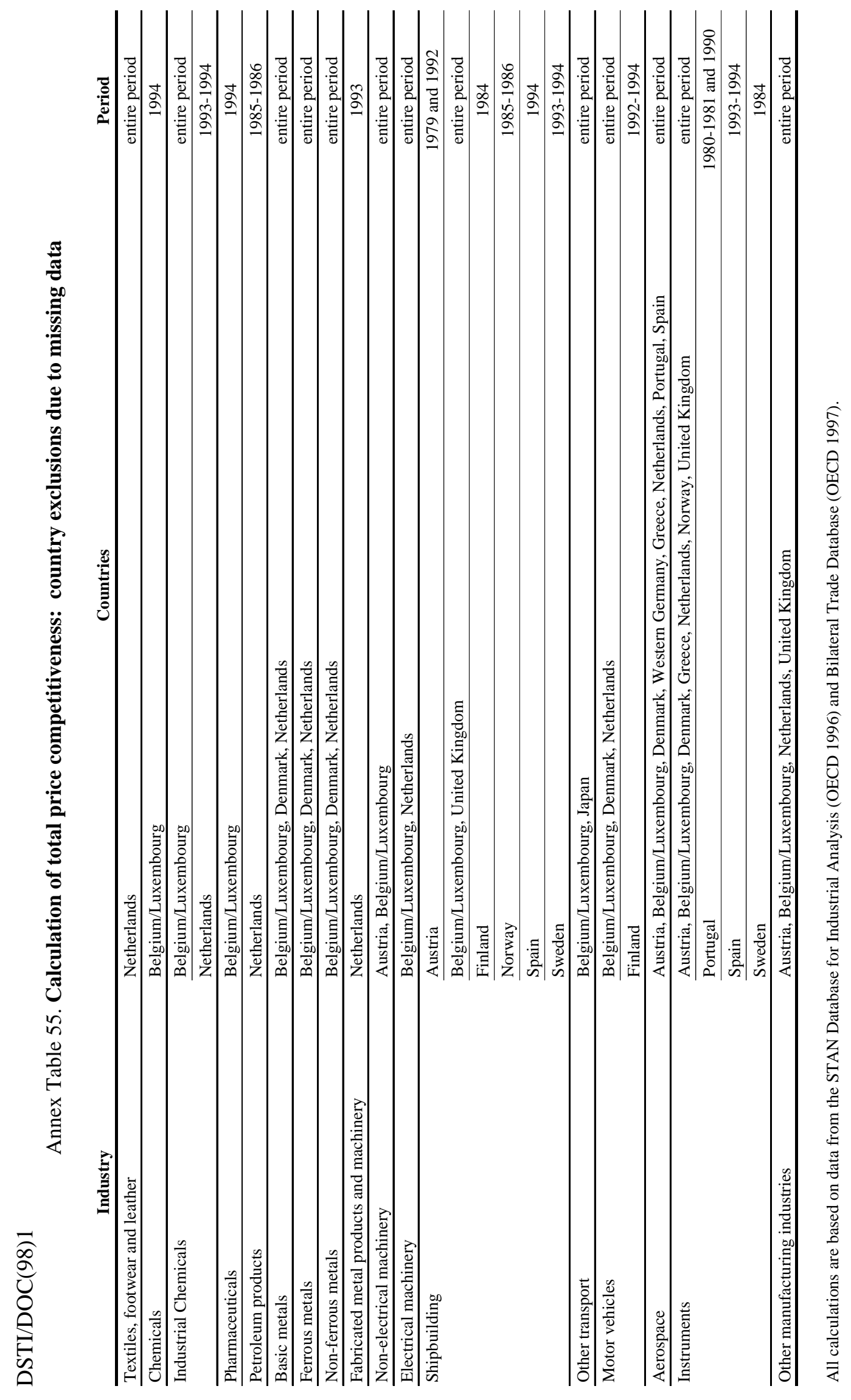




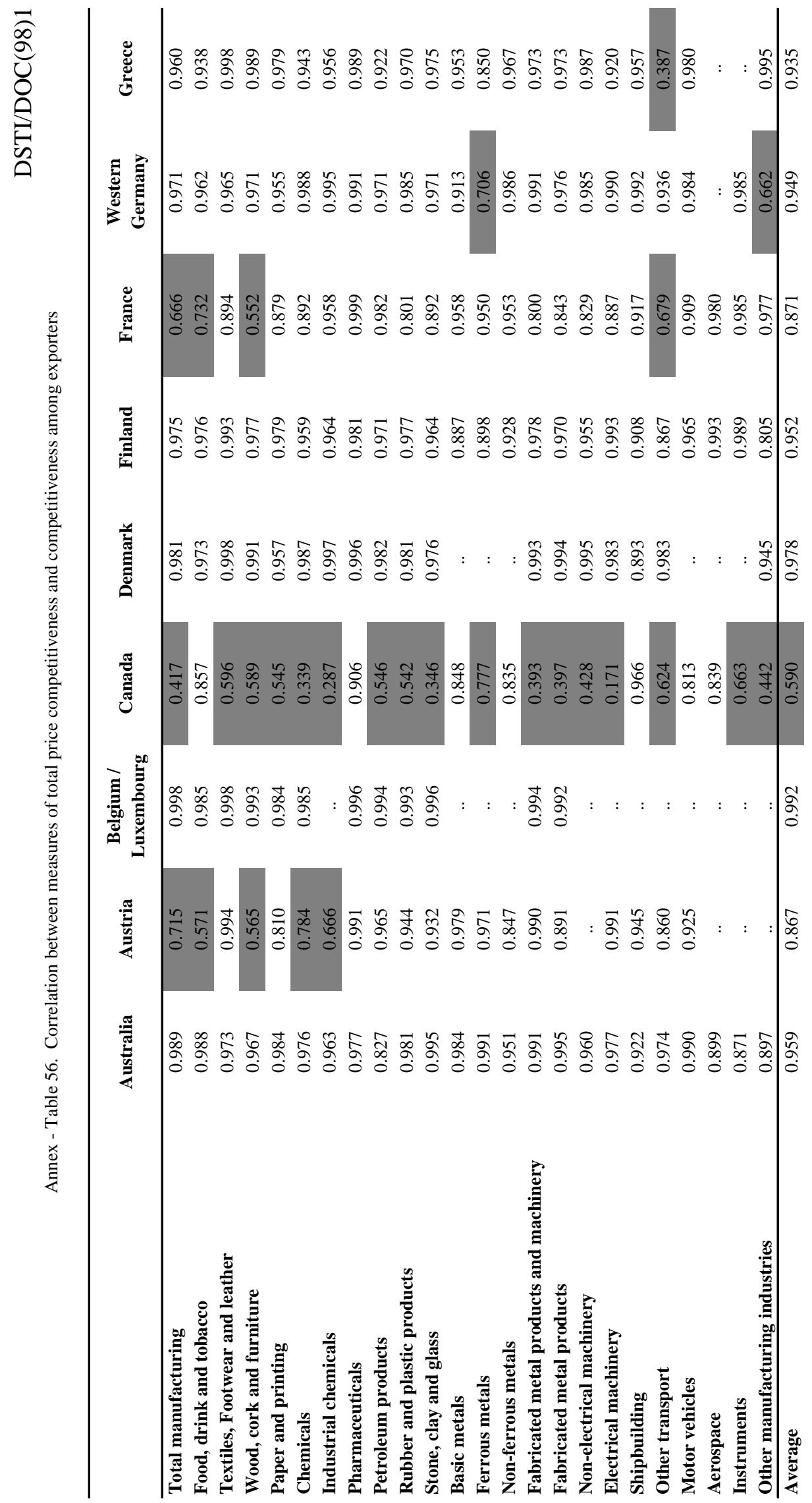




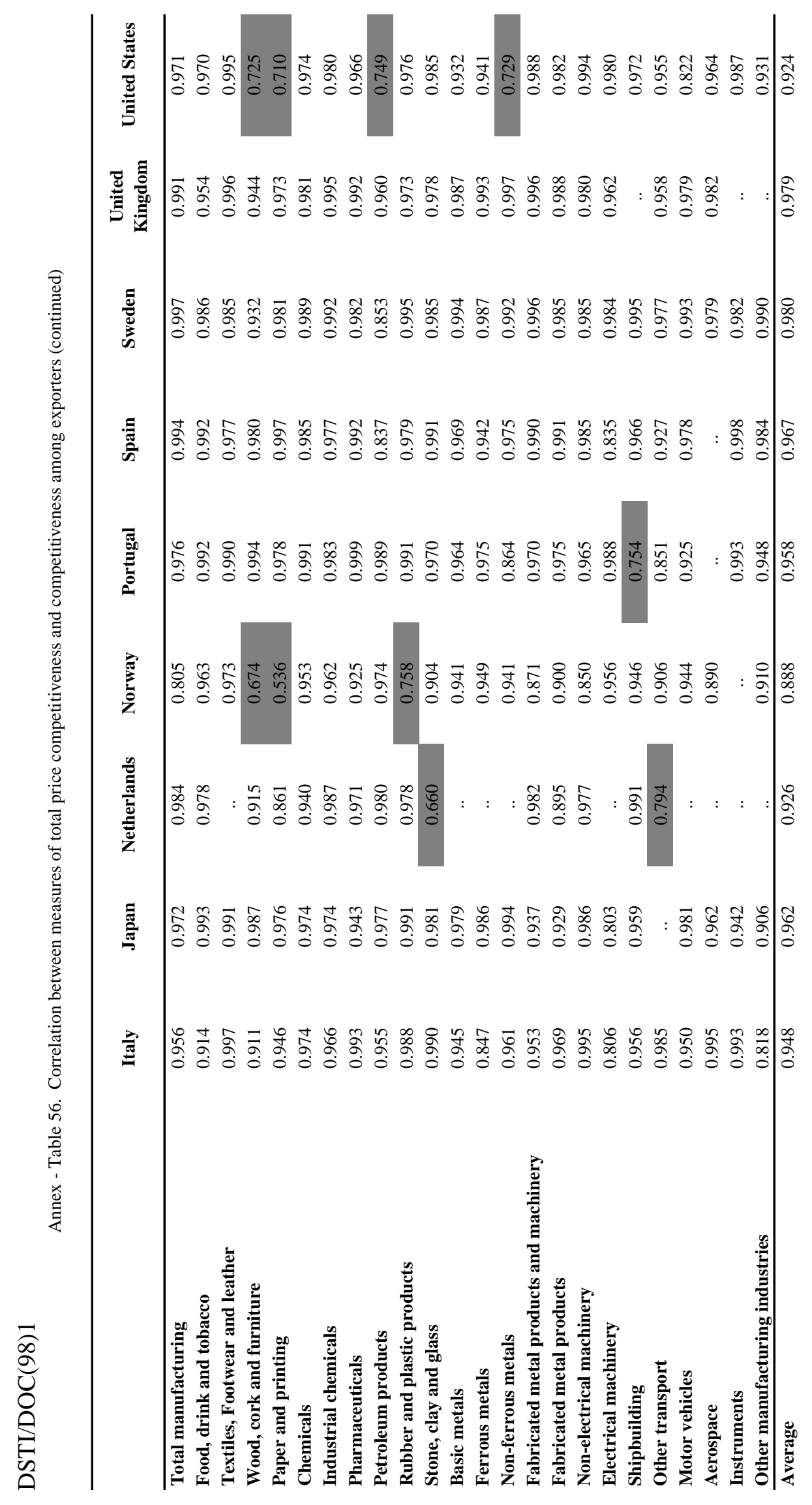




\title{
STI WORKING PAPERS
}

\author{
1996
}

1. Embodied Technology Diffusion: An Empirical Analysis for 10 OECD Countries George Papaconstantinou, Norihisa Sakurai and Andrew Wyckoff

2. The Impact of R\&D and Technology Diffusion on Productivity Growth: Evidence for 10 OECD Countries in the 1970s and 1980s

Norihisa Sakurai, Evangelos Ioannidis and George Papaconstantinou

3. Short-term Indicators: Using Qualitative Indicators to Update Production Indices Paul Schreyer and Corinne Emery

4. SMEs and Employment Creation: Overview of Selected Quantitative Studies in OECD Member Countries

Paul Schreyer

5. Globalisation and Competitiveness: Relevant Indicators

Thomas Hatzichronoglou

6. Factors Influencing the Steel Work Force: 1990 to 1995

Donald F. Barnett

7. Measuring R\&D in the Services

Alison Young

8. The Evolution of Skills in OECD Countries and the Role of Technology

A. Colecchia and G. Papaconstantinou

1997

1. Bibliometric Indicators and Analysis of Research Systems: Methods and Examples

(disponible en français sous le titre "Indicateurs bibliométriques et analyse des systèmes de recherche : méthodes et exemples")

Yoshiko Okubo

2. Revision of the High-technology Sector and Product Classification

(disponible en français sous le titre "Révision des classifications des secteurs et des produits de haute technologie")

Thomas Hatzichronoglou

\section{8}

1. Relative Trade-weighted Unit Labour Costs by Industry

Karine Lepron and Paul Schreyer 